\title{
Article \\ Optimizing Land Use and Land Cover Allocation for Flood Mitigation Using Land Use Change and Hydrological Models with Goal Programming, Chaiyaphum, Thailand
}

\author{
Athiwat Phinyoyang (D) and Suwit Ongsomwang *(D) \\ School of Geoinformatics, Institute of Science, Suranaree University of Technology, \\ Nakhon Ratchasima 30000, Thailand; phinyoyang.a@gmail.com \\ * Correspondence: suwit@sut.ac.th; Tel.: +66-8-9895-8149
}

check for

updates

Citation: Phinyoyang, A.; Ongsomwang, S. Optimizing Land Use and Land Cover Allocation for Flood Mitigation Using Land Use Change and Hydrological Models with Goal Programming, Chaiyaphum, Thailand. Land 2021, 10 , 1317. https://doi.org/10.3390/ land10121317

Academic Editor: Alexis Comber

Received: 19 October 2021

Accepted: 27 November 2021

Published: 30 November 2021

Publisher's Note: MDPI stays neutral with regard to jurisdictional claims in published maps and institutional affiliations.

Copyright: (c) 2021 by the authors. Licensee MDPI, Basel, Switzerland. This article is an open access article distributed under the terms and conditions of the Creative Commons Attribution (CC BY) license (https:// creativecommons.org/licenses/by/ $4.0 /)$.
Abstract: Floods represent one of the most severe natural disasters threatening the development of human society worldwide, including in Thailand. In recent decades, Chaiyaphum province has experienced a problem with flooding almost every year. In particular, the flood in 2010 caused property damage of 495 million Baht, more than 322,000 persons were affected, and approximately $1046.4 \mathrm{~km}^{2}$ of productive agricultural area was affected. Therefore, this study examined how to optimize land use and land cover allocation for flood mitigation using land use change and hydrological models with optimization methods. This research aimed to allocate land use and land cover (LULC) to minimize the surface for flood mitigation in Mueang Chaiyaphum district, Chaiyaphum province, Thailand. The research methodology consisted of six stages: data collection and preparation, LULC classification, LULC prediction, surface runoff estimation, the optimization of LULC allocation for flood mitigation and mapping, and economic and ecosystem service value evaluation and change. According to the results of the optimization and mapping of suitable LULC allocation to minimize surface runoff for flood mitigation in dry, normal, and wet years using goal programming and the CLUE-S model, the suitable LULC allocation for flood mitigation in 2049 under a normal year could provide the highest future economic value and gain. In the meantime, the suitable LULC allocation for flood mitigation in 2049 under a drought year could provide the highest ecosystem service value and gain. Nevertheless, considering future economic and ecosystem service values and changes with surface runoff reduction, the most suitable LULC allocation for flood mitigation is a normal year. Consequently, it can be concluded that the derived results of this study can be used as primary information for flood mitigation project implementation. Additionally, the presented conceptual framework and research workflows can be used as a guideline for government agencies to examine other flood-prone areas for flood mitigation in Thailand.

Keywords: optimizing land use and land cover allocation; surface runoff coefficient; goal programming; random forests; SCS-CN model; CLUE-S model; Chaiyaphum province; Thailand

\section{Introduction}

Floods represent one of the most severe natural disasters threatening the development of human society worldwide, including in Thailand. They cause enormous losses to economies, societies, and ecological environments [1], and the flood-related damage to agriculture and other related activities impacts a country's economy and development [2]

In general, the primary cause of flooding is heavy rainfall [3]. However, many other causes are also due to human activities, such as land degradation; deforestation of catchment areas, urban growth, and increased population along riverbanks [4-6]; poor land use planning, zoning, and control of flood plain development; poor drainage, particularly in cities; and insufficient management of discharge from river reservoirs [7].

In the last two decades, Chaiyaphum province has experienced a problem with flooding almost every year, causing a loss of lives, as well as economic losses, asset or 
housing losses, inundated farmlands, and decreased crop productivity for people who live in this area. In particular, the flood in 2010 caused property damage of 495 million Baht. More than 322,000 persons were affected, at least seven persons lost their lives, and approximately $1046.4 \mathrm{~km}^{2}$ of productive agricultural area was affected [8].

Due to the risk of large-scale damage to public and private property in Chaiyaphum province, the Royal Thai Government has allocated a significant budget to mitigate flood effects using structural measures, such as channel modification, bank protection, dikes, and reservoir development. However, the problems persist and are becoming exacerbated [9]. However, it is difficult to fundamentally mitigate flood damage using only flood prevention facilities [10]. A comprehensive flood control measure should consider land use and land cover change and optimum land use allocation.

In general, LULC strongly influences flood risk and affects the probability of floods and their consequences in several ways [11]. LULC change can affect the hydrological characteristics of a river basin through the influence of land use on runoff generation processes $[12,13]$. This study chose the SCS-CN method, which represents a distributed hydrologic model, to estimate the time series surface runoff according to LULC changes in the study period (2001-2019). These changes may alter the quantity of surface/subsurface runoff generation, river flooding regimes, and the extent [14]. Thus, defining optimal strategies for appropriate flood management, especially LULC management, is very important and necessary [15] for flood mitigation in Chaiyaphum province.

Land use optimization is one of the proper solutions for soil and water conservation at the watershed level. It can help decision-makers determine the best scenario of various land use alternatives without sacrificing the economic value obtained from the available land use [16,17]. Land use arrangement can be optimized using a programming model to increase land use earnings and reduce environmental impacts, especially surface runoff [16]. The essence of management science, manifested in modeling and programming techniques, is considered an essential tool for optimally allocating rare resources to gain the most benefits [18].

In recent decades, the new programming methods that have been developed can be employed under conflicting conditions of the goals and limited resources for decisionmakers. In natural resource management, there are many optimization techniques. Some approaches such as linear programming (LP), goal programming (GP), and weighted goal programming (WGP) are widely employed in land use optimization at the watershed level [11]. For instance, Yeo et al. applied LP to optimize land use to peak discharge minimization at the Old Woman Cheek watershed, Ohio State, USA [19]. Owji et al. applied LP for land use optimization in the Jajrood watershed, Iran, to reduce surface runoff and sediment yield [20]. Likewise, Aldea et al. used GP for forest management in the Pinar Grande Forest, Spain [21]. Further, Gonfa and Kumar applied LP and GP for optimum land use to minimize soil erosion and maximize the net benefit in Ethiopia's Mojo watershed [22], and Al-Zahrani et al. developed GP for optimizing water resources in Riyadh, Saudi Arabia [23]. Similarly, Sokouti and Nikkami applied LP to optimize land use patterns to reduce soil erosion in West Azerbaijan province, Iran [24]. WGP has been applied to optimize LULC allocation for surface runoff and sediment load minimization at Bayg watershed [11]. Moreover, LP has been used to maximize cropland allocation in Abaro Kebele, Ethiopia [25]. Recently, Han et al. applied LP to optimize the land use structure for carbon emission reduction in Shenzhen, China [26].

Nevertheless, the integration of the optimization technique (GP), advanced land use change modeling (CLUE-S model), and the distributed hydrological model (SCS-CN model) to minimize surface runoff for flood mitigation does not exist in Thailand. Therefore, a novel classification method, random forests, was first applied to classify LULC data in 2001, 2010, and 2019. Then, the classified data were further used to predict a time series LULC between 2001 and 2019 using the CLUE-S model for time series surface runoff estimation using the SCS-CN model. After this, goal programming was applied to minimize surface runoff for flood mitigation based on the surface runoff coefficient value of each LULC type 
in 2029, 2039, and 2049 in dry, normal, and wet years. Finally, economic and ecosystem service value change between the existing LULC data in 2019 and the suitable LULC allocation in dry, normal, and wet years was examined in terms of gain and loss using the present value (PV) model and the simple benefit transfer method.

The specific objectives of the study were (1) to classify LULC data in 2001, 2010, and 2019 using the random forest classifier, (2) to predict LULC change in two periods (2002-2009 and 2011-2018) using the CLLUE-S model, (3) to estimate surface runoff between 2001 and 2019, (4) to optimize and map LULC allocation for flood mitigation under three rainfall conditions, and (5) to evaluate economic and ecosystem service values and change for the most suitable LULC allocation for flood mitigation.

\section{Study Area}

The study area was the Second Part of the Lam Nam Chi watershed, Chaiyaphum province, Thailand, under the Chi River basin, covering approximately $3794 \mathrm{~km}^{2}$. As mentioned earlier, the selected study area covers the flood-prone area in Mueang Chaiyaphum district, Chaiyaphum province. The topography of the area is generally characterized by rolling hilly terrain and flat areas. The elevation ranges from $162 \mathrm{~m}$ above the mean sea level (MSL) in the lower part of the watershed to approximately $1034 \mathrm{~m}$ above MSL in the upper part of the watershed (Figure 1). The study area consists of nine soil groups: clay, clay loam, loam, loamy sand, sandy loam, sandy clay loam, silty clay, silty loam, and silty clay loam [27]. Meanwhile, the top three dominant land use types in 2015 were paddy fields $(43.47 \%)$, cassava $(12.69 \%)$, and forest land $(12.48 \%)$ [28].

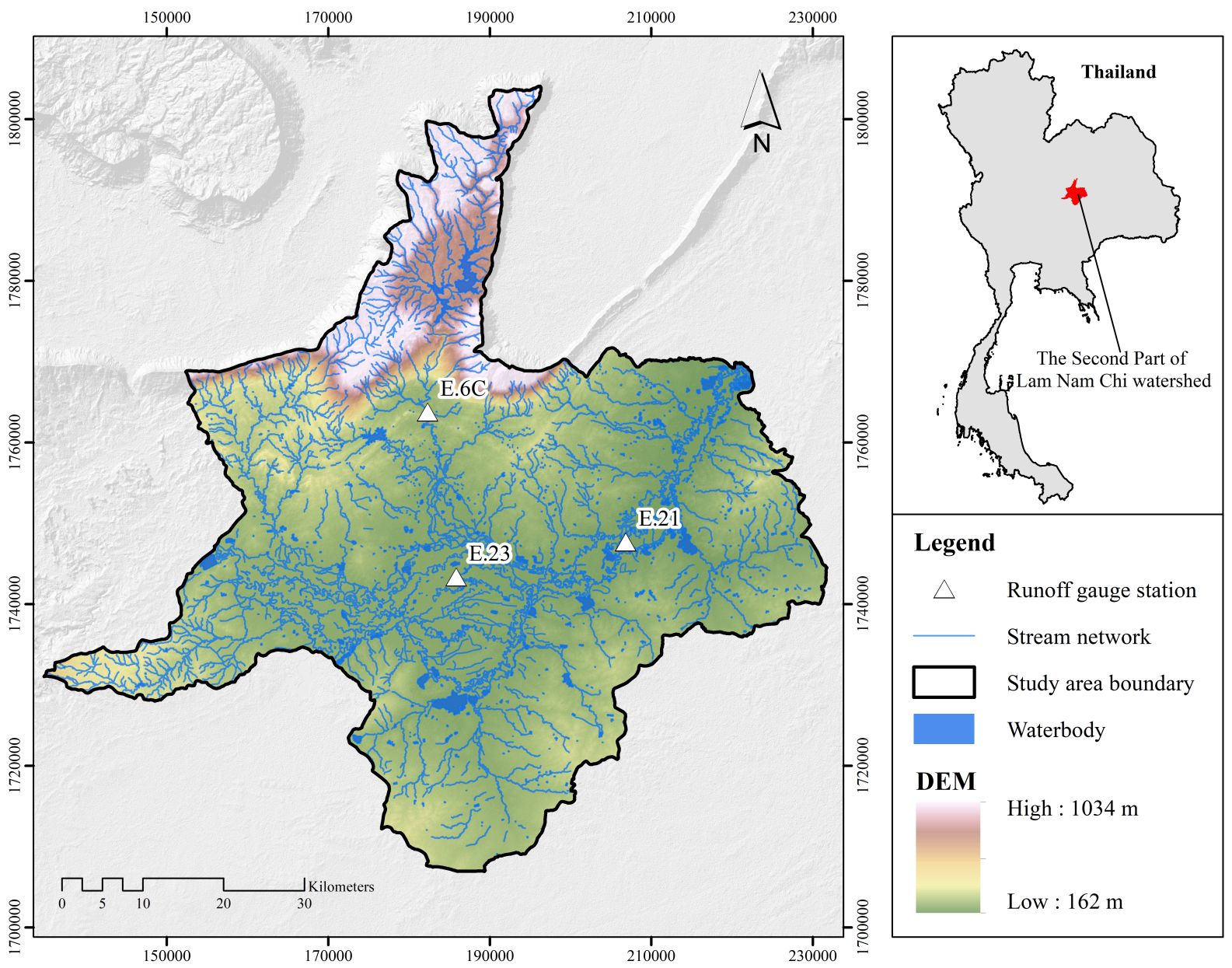

Figure 1. Terrain characteristics of the study area with runoff gauge stations. 


\section{Materials and Methods}

The research methodology consisted of data collection and preparation and five significant components, which were (1) LULC classification, (2) LULC prediction, (3) surface runoff estimation, (4) optimization of LULC allocation for flood mitigation and mapping, and (5) economic and ecosystem service value evaluation and change (Figure 2). Details of each stage are separately described in the following sections.

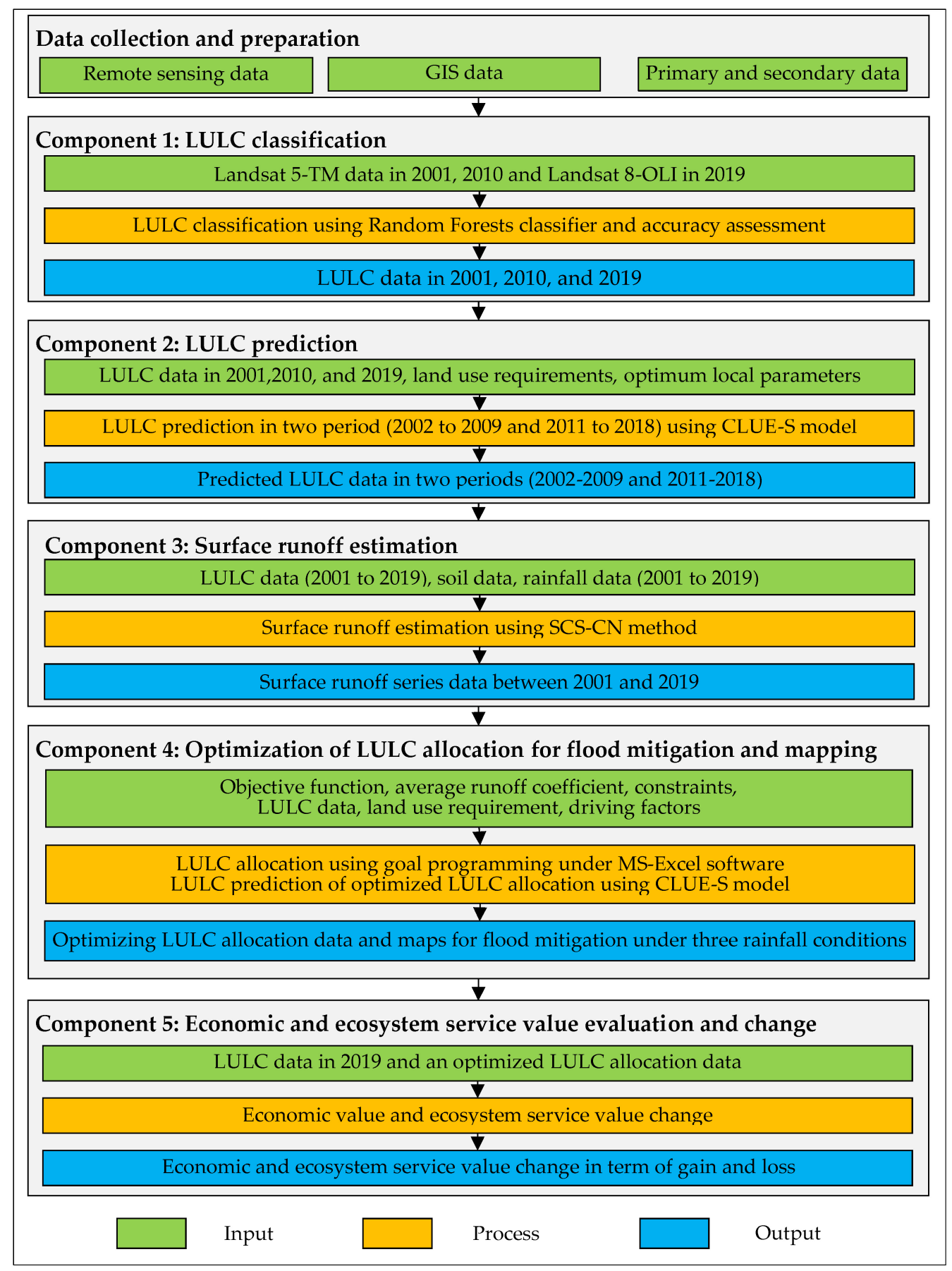

Figure 2. Workflow of the research methodology.

\subsection{Data Collection and Preparation}

The required input data for data analysis included GIS data, remote sensing data, and primary and secondary data, which were collected and prepared, as summarized in Table 1. 
Table 1. List of data collection and preparation for data analysis in this study.

\begin{tabular}{|c|c|c|c|}
\hline Data & Data Collection & Data Preparation & Source \\
\hline \multirow[t]{2}{*}{ Primary } & Ground reference & - & - \\
\hline & Runoff & - & RID \\
\hline \multirow{3}{*}{ Secondary } & Annual rainfall & Interpolation & TMD \\
\hline & & Population density & DOPA \\
\hline & Socioeconomic data & Income per capita & NESDC \\
\hline \multirow{11}{*}{ Remote Sensing } & Landsat 5 TM: Path 128 Row 49, 6 January 2001 & & \\
\hline & Landsat 5 TM: Path 129 Row 49, 14 February 2001 & & \\
\hline & Landsat 5 TM: Path 128 Row 49, 16 February 2010 & - & \\
\hline & Landsat 5 TM: Path 129 Row 49, 23 February 2010 & - & USGS \\
\hline & Landsat 8 OLI: Path 128 Row 49, 24 January 2019 & & \\
\hline & Landsat 8 OLI: Path 129 Row 49, 31 January 2019 & & \\
\hline & Satellite image from Google Earth in 2010 & - & Google \\
\hline & Color orthophotograph & - & RTSD \\
\hline & Administrative boundary & - & DEQP \\
\hline & Soil (soil series) & Recode & LDD \\
\hline & Watershed boundary & - & RID \\
\hline \multirow{5}{*}{ GIS } & Elevation & Extract from DEM & SRTM \\
\hline & Slope & Extract from DEM & SRTM \\
\hline & Road network & Buffering & MOT, DEQP \\
\hline & Stream & Buffering & RTSD \\
\hline & Urban area & Buffering & LULC data \\
\hline
\end{tabular}

Note: USGS, United States Geological Survey; RTSD, Royal Thai Survey Department; DEQP, Department of Environmental Quality Promotion; TMD, Thai Meteorological Department; RID, Royal Irrigation Department; LDD, Land Development Department; NESDC, Office of the National Economic and Social Development Council; SRTM, Shuttle Radar Topography Mission; MOT, Ministry of Transport; DOPA, Department of Provincial Administration.

\subsection{LULC Classification}

Landsat imageries in 2001, 2010, and 2019 were downloaded from the USGS website (www.earthexplore.usgs.gov, accessed on 22 November 2021) for LULC classification using the RF classifier of the EnMap-Box software. In practice, the training areas of each LULC type in a specific year were separately prepared to extract multiple decision trees for LULC classification. Spectral reflectance data (visible, NIR, and SWIR bands), additional spectral bands, and elevation were applied to classify the LULC types. The spectral bands that enhance particular features for LULC classification include the Normalized Difference Vegetation Index (NDVI) to represent vegetation features [29], the Modified Normalized Difference Wetness Index (MNDWI) to signify the moisture regime [30], and the Normalized Difference Built-up Index (NDBI) to indicate built-up areas [31]. Likewise, elevation is directly related to the spatial distribution of LULC type, e.g., paddy fields are generally situated in the floodplain, while forests are primarily located in mountainous areas.

In this study, the modified land use classification of the LDD consisted of (1) urban and built-up areas, (2) paddy fields, (3) sugarcane, (4) cassava, (5) other field crops, (6) para rubber, (7) perennial trees and orchards, (8) forest land, (9) waterbodies, (10) rangeland, (11) marshes and swamps, and (12) unused land.

After classification, the LULC maps in 2001, 2010, and 2019 were assessed for thematic accuracy (overall accuracy and Kappa hat coefficient) based on the reference data from color orthophotograph in 2000-2001, very high spatial resolution imageries from Google Earth in 2010, and field surveys in 2020, respectively. This study estimated the number of sample sizes for thematic accuracy assessment based on multinomial distribution with a stratified random sampling scheme, as suggested by [32].

\subsection{LULC Prediction}

The CLUE-S model was selected to predict LULC data in two periods, 2002-2009 and 2011-2018, for filling the gap of LULC data between three classified LULC data in 2001, 
2010, and 2019. As a result, time-series LULC data between 2001 and 2019 will be available for annual surface runoff estimation in this study.

3.3.1. Optimal Local Driving Factors on Land Use Change Identification for LULC Prediction

The land use change model CLUE-S was selected to predict the LULC data in two periods, 2002-2009 and 2011-2018, for data analysis. The local driving factors on land use change for LULC prediction were identified by comparing the predicted LULC map in 2019 with the classified LULC map in 2019. The basic parameters of the CLUE-S model, which include (1) elasticity value, (2) LULC conversion matrix, and (3) land requirement of each LULC type in 2019, were firstly extracted based on the final LULC map in 2001 and 2010 using the Markov Chain model. At the same time, the selected three driving factor categories on LULC change, including physical, socioeconomic, and proximity data, which were reviewed from the previous studies of many researchers [33-45], were examined multicollinearity, and significant driving factors for LULC identified by allocating using binomial logistic regression analysis, as follows:

$$
\log \left(\frac{P_{i}}{1-P_{i}}\right)=\beta_{0}+\beta_{1} X_{1, i}+\beta_{2} X_{2, i} \ldots \ldots+\beta_{n} X_{n, i}
$$

where $P_{i}$ is the probability of a grid cell for the considered land use type on location $i$, and the Xs are the location factors. The coefficients $(\beta)$ were estimated through logistic regression using the actual land use pattern as the dependent variable [46].

After this, the predicted LULC map in 2019 was compared to the classified map in 2019 using a wall-to-wall thematic accuracy assessment with overall accuracy and Kappa hat coefficient. If the overall accuracy and Kappa hat coefficient were equal to or more than $80 \%$, then the derived significant driving factors by binomial logistic regression analysis were chosen as the optimal local driving factors on land use change for LULC prediction using the CLUE-S model.

\subsubsection{LULC Prediction of Two Periods: 2002-2009 and 2011-2018}

The optimal local driving factors on land use change for LULC prediction, namely, elasticity value, LULC conversion matrix, and land requirement of each LULC type in two time periods (2002-2009 and 2011-2018), which were extracted using the Markov Chain model based on the corresponding LULC data in 2001, 2010, and 2019, were applied to predict the LULC data in the two periods using the CLUE-S model.

\subsection{Surface Runoff Estimation}

This study estimated the time series surface runoff between 2001 and 2019 based on the classified and predicted LULC data, soil series, and rainfall data using the SCS-CN method with suitable AMC via ESRI ArcGIS software.

In practice, the required input data included LULC, soil series, rainfall, and hydrologic soil group data, prepared and operated for surface runoff estimation using the SCS-CN method in raster format with a cell size of $30 \mathrm{~m}$ in a raster-based GIS environment. The surface runoff depth in each cell was semi-automatically generated based on runoff curve numbers (CNs) according to hydrologic soil group-land cover complex using the Model Builder of ArcGIS, as shown in Figure 3. 


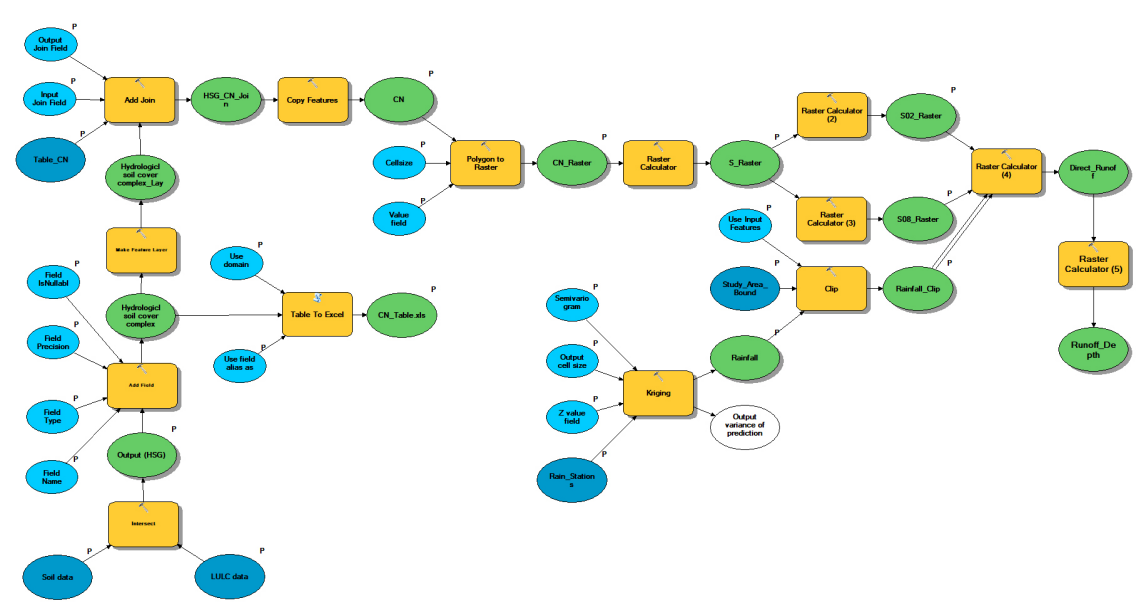

Figure 3. Schematic diagram of Model Builder for surface runoff estimation.

The soil series data, which were applied to classify soil texture classes based on the percentage of sand, silt, and clay of each soil series unit, are displayed in Figure 4a. Meanwhile, the hydrologic soil group (HSG), which presents potential runoff in the study area according to soil texture classes, is shown in Figure $4 \mathrm{~b}$.

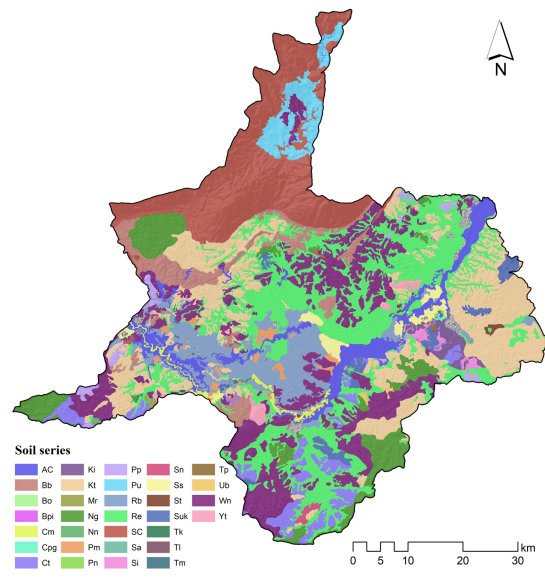

(a)

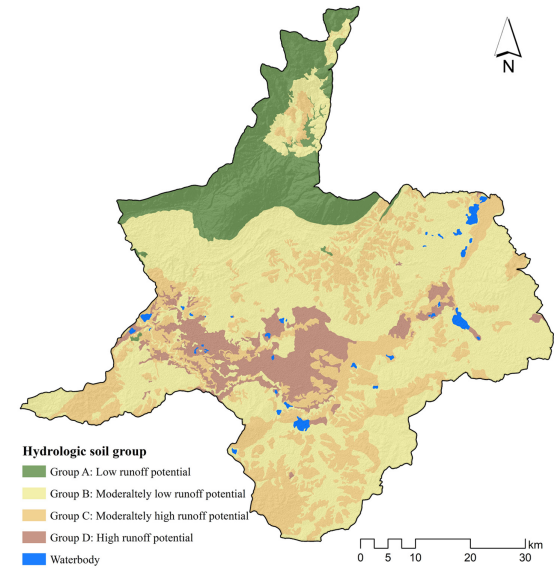

(b)

Figure 4. Spatial distribution of (a) soil series and (b) hydrologic soil group.

In this study, two significant steps implemented under this component include (1) suitable AMC for surface runoff estimation using the SCS-CN method and (2) surface runoff estimation between 2011 and 2019.

\subsubsection{Suitable AMC for Surface Runoff Estimation Using the SCS-CN Method}

The suitable AMC condition for surface runoff estimation using the SCS-CN method was examined based on the classified and predicted LULC data between 2001 and 2010 with three different $C N$ values of three different AMCs, as suggested by [47] using the following equations:

$$
\begin{aligned}
& \mathrm{CN}_{\mathrm{I}}=\frac{4.2 \mathrm{CN}_{\mathrm{II}}}{10-0.058 \mathrm{CN}_{\mathrm{II}}} \\
& \mathrm{CN}_{\mathrm{III}}=\frac{23 \mathrm{CN}_{\mathrm{II}}}{10+0.13 \mathrm{CN}_{\mathrm{II}}}
\end{aligned}
$$

where $\mathrm{CN}_{\mathrm{I}}$ is the runoff curve number value of each LULC type of AMC-I, $\mathrm{CN}_{\mathrm{II}}$ is the runoff curve number value of each LULC type of AMC-II, and $\mathrm{CN}_{\text {III }}$ is the runoff curve number value of each LULC type of AMC-III (Table 2). 
Table 2. Runoff curve number under AMC-I, -II, and -III.

\begin{tabular}{|c|c|c|c|c|c|c|c|c|c|c|c|c|}
\hline \multirow{2}{*}{ LULC Types } & \multicolumn{4}{|c|}{ CN Value of AMC-I } & \multicolumn{4}{|c|}{ CN Value of AMC-II } & \multicolumn{4}{|c|}{ CN Value of AMC-III } \\
\hline & A & B & $\mathrm{C}$ & $\mathbf{D}$ & $\mathbf{A}$ & B & C & D & A & B & $\mathrm{C}$ & D \\
\hline Urban and built-up area & 77.26 & 82.85 & 86.81 & 88.86 & 89 & 92 & 94 & 95 & 94.9 & 96.36 & 97.3 & 97.76 \\
\hline Cassava and other field crops & 51.92 & 64.16 & 75.49 & 80.94 & 72 & 81 & 88 & 91 & 85.54 & 90.75 & 94.4 & 95.88 \\
\hline Sugarcane & 28.75 & 48.32 & 61.24 & 68.8 & 49 & 69 & 79 & 84 & 68.85 & 83.66 & 89.64 & 92.35 \\
\hline Paddy fields & 43.82 & 57.08 & 68.8 & 75.49 & 65 & 76 & 84 & 88 & 81.03 & 87.93 & 92.35 & 94.4 \\
\hline Rangeland & 28.75 & 48.32 & 61.24 & 68.8 & 49 & 69 & 79 & 84 & 68.85 & 83.66 & 89.64 & 92.35 \\
\hline $\begin{array}{l}\text { Para rubber and perennial } \\
\text { trees and orchards }\end{array}$ & 24.06 & 43.82 & 57.08 & 65.68 & 43 & 65 & 76 & 82 & 63.44 & 81.03 & 87.93 & 91.29 \\
\hline Forest land & 15.25 & 33.92 & 49.49 & 58.44 & 30 & 55 & 70 & 77 & 49.64 & 73.76 & 84.29 & 88.51 \\
\hline $\begin{array}{l}\text { Waterbodies and marshes } \\
\text { and swamps }\end{array}$ & 95.37 & 95.37 & 95.37 & 95.37 & 98 & 98 & 98 & 98 & 99.12 & 99.12 & 99.12 & 99.12 \\
\hline Unused land & 58.44 & 72.07 & 80.94 & 86.81 & 77 & 86 & 91 & 94 & 88.51 & 93.39 & 95.88 & 97.3 \\
\hline
\end{tabular}

In practice, the three runoff curve numbers of the hydrologic soil group of each LULC type were separately applied to estimate the potential maximum storage under three different AMCs using Equation (4).

$$
\mathrm{S}=25.4 \frac{1000}{\mathrm{CN}}-10
$$

where $\mathrm{CN}$ is the runoff curve number of the hydrologic soil group (HSG)-land cover complex.

The calculated potential maximum storage was further applied to estimate the surface runoff depth of the three different AMCs [48,49], as follows:

$$
\mathrm{Q}=\frac{(\mathrm{P}-0.2 \mathrm{~S})^{2}}{(\mathrm{P}+0.8 \mathrm{~S})}
$$

where $Q$ is the surface runoff depth $(\mathrm{mm}), \mathrm{P}$ is the annual rainfall $(\mathrm{mm})$, and $\mathrm{S}$ is the potential maximum storage.

Then, the estimated surface runoff depth of the three different AMCs from 2001 to 2010 was converted into surface runoff volume using Equation (6).

$$
\text { Surface runoff volume }=\frac{\text { Surface runoff depth }}{1000} \times \text { cell size }
$$

Later, they were used to identify the suitable AMC using model performance scale, including Nash and Sutcliffe's coefficient of efficiency (NSE), the coefficient of determination $\left(R^{2}\right)$, and the percent of bias (PBIAS) (Equations (7)-(9)), as suggested by [50] (Table 3).

$$
\mathrm{NSE}=1-\left[\frac{\sum_{\mathrm{i}}^{\mathrm{n}}\left(\mathrm{Q}_{\text {simi }}-\mathrm{Q}_{\mathrm{obsi}}\right)^{2}}{\sum_{\mathrm{i}}^{\mathrm{n}}\left(\mathrm{Q}_{\mathrm{obsi}}-\mathrm{Q}_{\mathrm{avg}}\right)^{2}}\right]
$$

where $n$ is the number of years, $Q_{\text {simi }}$ is the simulated surface runoff, $Q_{o b s i}$ is the observed surface runoff, and $Q_{a v g}$ is the average observed surface runoff over the simulation period. The values for $\mathrm{E}$ can vary from $-\infty$ to 1 , with 1 indicating a perfect fit.

$$
\mathrm{R}^{2}=\left\{\frac{\sum_{\mathrm{i}=1}^{\mathrm{n}}\left(\mathrm{Q}_{\text {obsi }}-\mathrm{Q}_{\text {obsavg }}\right)\left(\mathrm{Q}_{\text {simi }}-\mathrm{Q}_{\text {simavg }}\right)}{\left[\sum_{\mathrm{i}=1}^{\mathrm{n}}\left(\mathrm{Q}_{\mathrm{obsi}}-\mathrm{Q}_{\mathrm{obsavg}}\right)^{2} \sum_{\mathrm{i}=1}^{\mathrm{n}}\left(\mathrm{Q}_{\text {simi }}-\mathrm{Q}_{\text {simavg }}\right)^{2}\right]^{0.5}}\right\}^{2}
$$


where $Q_{\text {obsi }}$ is the observed surface runoff in year $i, Q_{\text {simi }}$ is the simulated surface runoff in year $i, Q_{\text {obsavg }}$ is the average of the observed surface runoff over the calibration or validation period, $\mathrm{Q}_{\text {simavg }}$ is the average of the simulated surface runoff over the validation period, $i$ is the year, and $n$ is the total count of data pairs.

$$
\text { PBIAS }=\left[\frac{\sum_{\mathrm{i}=1}^{\mathrm{n}}\left(\mathrm{Y}_{\mathrm{i}}^{\text {obs }}-\mathrm{Y}_{\mathrm{i}}^{\text {sim }}\right) \times(100)}{\sum_{\mathrm{i}=1}^{\mathrm{n}} \mathrm{Y}_{\mathrm{i}}^{\text {obs }}}\right]
$$

where $Y_{i}^{\text {obs }}$ is the observed surface runoff in time step $i$, and $Y_{i}^{\text {sim }}$ is the simulated surface runoff in time step $i$.

Table 3. Model performance scale.

\begin{tabular}{ccccc}
\hline \multirow{2}{*}{$\begin{array}{c}\text { Statistics } \\
\text { Measurement }\end{array}$} & \multicolumn{4}{c}{ Performance Ratings } \\
\cline { 2 - 5 } & Unsatisfactory & Satisfactory & Good & Very Good \\
\hline NSE & $<0.5$ & $0.5-0.65$ & $0.65-0.75$ & $0.75-1$ \\
$\mathrm{R}^{2}$ & $<0.5$ & $0.5-0.6$ & $0.6-0.7$ & $0.7-1$ \\
PBIAS & $>25$ & $15-25$ & $10-15$ & $<10$ \\
\hline
\end{tabular}

In this study, the observed runoff data between 2001 and 2010 from the hydrological station at E.21, E.23, and E.6C of the RID were used to calculate an average NSE, $\mathrm{R}^{2}$, and PBIAS for suitable AMC identification (see the location of the station in Figure 1).

\subsubsection{Surface Runoff Estimation between 2011 and 2019}

The runoff curve number of the suitable AMC was applied to estimate the surface runoff between 2011 and 2019 based on the classified and predicted LULC data in the same period. The estimated surface runoff data between 2011 and 2019 were further examined for model validation based on the observed runoff data in the same period from the same gauges of the RID using NSE, $\mathrm{R}^{2}$, and PBIAS.

The time series surface runoff estimation (between 2001 and 2019) from two steps will be further applied to extract the average runoff coefficient of each LULC type of three rainfall conditions for optimizing LULC allocation for flood mitigation in the next component.

\subsection{Optimization of LULC Allocation for Flood Mitigation and Mapping}

Goal programming was first applied to optimize LULC allocation for flood mitigation under dry, normal, and wet years. Then, the suitable LULC allocation data of dry, normal, and wet years were mapped using the CLUE-S model.

\subsubsection{SPI Calculation for the Rainfall Condition Identification}

Annual rainfall data between 1987 and 2019 were first used to calculate the 12-month SPI values. Then, they were reclassified into three rainfall conditions according to the SPI drought classification of [51] as follows:

1. If any year had a 12 -month SPI value less than or equal to -0.50 , the annual rainfall was categorized as a dry year;

2. If any year had a 12 -month SPI value between -0.49 and 0.49 , the annual rainfall was categorized as a normal year;

3. If any year had a 12-month SPI value more than or equal to 0.50 , the annual rainfall was categorized as a wet year.

After that, the average surface runoff coefficient for each LULC type of three rainfall conditions (dry, normal, and wet years) was extracted from the time series surface runoff and LULC data using zonal statistical analysis for optimization of LULC allocation to minimize surface runoff for flood mitigation. 


\subsubsection{Optimization of LULC Allocation to Minimize Surface Runoff for Flood Mitigation}

This study assigned the constraint sets for optimizing LULC allocation in 2029, 2039, and 2049 using the Markov Chain model based on the historical LULC development between 2010 and 2019. The changing area of each LULC type was considered according to the derived transitional change area from the Markov Chain model. Then, the derived average runoff coefficient and constraints of the objective function were applied to optimize LULC allocation to minimize surface runoff for flood mitigation under dry, normal, and wet years using goal programming with "What's Best!" as an extension program in an MS Excel environment.

The goal programming model, working as the surface runoff minimization function, can be expressed as the following equations:

Minimize surface runoff:

$$
\operatorname{Min}(z)=\sum_{i=1}^{n} C_{i} X_{i}
$$

Subject to constraints:

$$
\begin{gathered}
\sum_{i=1}^{n} x_{i}=A \\
\sum_{i=1}^{n} x_{i} \geq A \\
\sum_{i=1}^{n} x_{i} \leq A \\
X_{i} \geq 0
\end{gathered}
$$

where $\mathrm{Z}$ is the total annual surface runoff of the study area $\left(\mathrm{m}^{3} /\right.$ year), $\mathrm{C}_{\mathrm{i}}$ is the average surface runoff coefficient in each land use type $\left(\mathrm{m}^{3} / \mathrm{km}^{2} /\right.$ year $), X_{i}$ is the area of land use class $\mathrm{i}\left(\mathrm{km}^{2}\right), \mathrm{n}$ is the number of land use classes, and $\mathrm{A}$ is the total area of land use classes $\left(\mathrm{km}^{2}\right)$.

Finally, the suitable LULC allocation to minimize surface runoff for flood mitigation under dry, normal, and wet years was separately identified based on surface runoff reduction compared to the actual data in 2019.

\subsubsection{Mapping of Suitable LULC Allocation for Flood Mitigation}

The suitable LULC allocation to minimize surface runoff for flood mitigation under dry, normal, and wet years was separately mapped using the CLUE-S model. The required input data for LULC mapping included LULC data in 2019, local driving factors on land use change, elasticity value, LULC conversion matrix, and suitable LULC allocation data of three rainfall conditions as the land requirement.

\subsection{Economic and Ecosystem Service Value Evaluation and Change}

Economic and ecosystem service value evaluation and change were separately evaluated using the present value (PV) model and the simple benefit transfer method based on LULC data in 2019 and suitable LULC allocation data to minimize surface runoff for flood mitigation under dry, normal, and wet years in terms of gain and loss.

\subsubsection{Economic Value Evaluation and Change}

For economic value evaluation, the LULC data in 2019 and the suitable LULC allocation data to minimize surface runoff under dry, normal, and wet years were first calculated in terms of economic values using Equation (15), as suggested by [52]. Then, the future economic value changes between the LULC data in 2019 and the suitable LULC allocation 
data to minimize surface runoff under dry, normal, and wet years were compared using the image algebra change detection algorithm for gain and loss.

$$
\mathrm{PV}=\mathrm{FV} \cdot\left[\frac{100}{100+\mathrm{IR}}\right]^{\mathrm{Y}}
$$

where $\mathrm{PV}$ is the present value, $\mathrm{FV}$ is the future value, IR is the interest rate in percent, and $\mathrm{Y}$ is the number of years from the present, counting from zero.

\subsubsection{Ecosystem Service Value Evaluation and Change}

In general, ecosystem services represent a dynamic field in current scientific research, linking ecological, economic, and social aspects, demanding practical applications and methodologies at different spatial scales, and maintaining environmental management and decision-making processes [53-55].

This study applied a simple benefit transfer method [53] to evaluate the ecosystem service values of LULC data in 2019 and suitable LULC allocation data to minimize surface runoff under dry, normal, and wet years using Equation (16) with the coefficient value for different LULC types (Table 4). Then, the ecosystem service value change between the LULC data in 2019 and the suitable LULC allocation data to minimize surface runoff under dry, normal, and wet years was compared using the image algebra change detection algorithm for gain and loss.

$$
\mathrm{ESV}=\sum\left(\mathrm{A}_{\mathrm{k}} \times \mathrm{VC}_{\mathrm{k}}\right)
$$

where ESV denotes the total value of the ecosystem service, while $A_{k}$ and $V_{k}$ represent the area and value coefficient for proxy LULC type " $k$," respectively.

Table 4. LULC type and coefficient value for ESV evaluation.

\begin{tabular}{cccc}
\hline No. & LULC Classification for RF & $\begin{array}{c}\text { LULC Classification } \\
\text { for ESV }\end{array}$ & $\begin{array}{c}\text { Coefficient Values } \\
\text { (USD/ha/year) }\end{array}$ \\
\hline 1 & Urban and built-up areas & Construction land & 12.7 \\
2 & Paddy fields & Cultivated land & 1032.3 \\
3 & Field crops & Cultivated land & 1032.3 \\
4 & Para rubber & Forest land & 1949.0 \\
5 & Perennial trees and orchards & Forest land & 1949.0 \\
6 & Forest land & Forest land & 1949.0 \\
7 & Waterbodies & Waterbodies & 6873.7 \\
8 & Rangeland & Rangeland & 808.6 \\
9 & Wetland & Wetland & 9368.7 \\
\hline
\end{tabular}

${ }^{1}$ Modified from [56].

Finally, the future economic and ecosystem service values and their changes in suitable LULC allocation for flood mitigation by each rainfall condition (dry, normal, and wet years) were compared to justify the most suitable LULC allocation for flood mitigation among three rainfall conditions.

\section{Results and Discussion}

\subsection{Land Use and Land Cover Classification and Change Detection}

The results of the LULC classification in 2001, 2010, and 2019 using the random forests (RF) classifier based on Landsat imageries and supplementary data, including NDVI, MNDWI, NDBI, and DEM, are reported in Table 5 and displayed in Figure 5. 
Table 5. Area and percentage of LULC data in 2001, 2010, and 2019.

\begin{tabular}{|c|c|c|c|c|c|c|c|}
\hline \multirow{2}{*}{ No. } & \multirow{2}{*}{ LULC Types } & \multicolumn{2}{|c|}{ LULC 2001} & \multicolumn{2}{|c|}{ LULC 2010} & \multicolumn{2}{|c|}{ LULC 2019} \\
\hline & & Area $\left(\mathrm{km}^{2}\right)$ & Percentage & Area $\left(\mathrm{km}^{2}\right)$ & Percentage & Area $\left(\mathrm{km}^{2}\right)$ & Percentage \\
\hline 1 & Urban and built-up areas & 46.17 & 1.22 & 53.21 & 1.4 & 65.84 & 1.74 \\
\hline 2 & Paddy fields & 2344.39 & 61.79 & 2070.71 & 54.58 & 2012.16 & 53.03 \\
\hline 3 & Sugarcane & 61.25 & 1.61 & 153.52 & 4.05 & 306.85 & 8.09 \\
\hline 4 & Cassava & 532.95 & 14.05 & 629.33 & 16.59 & 489.91 & 12.91 \\
\hline 5 & Other field crops & 2.09 & 0.06 & 5.19 & 0.14 & 6.19 & 0.16 \\
\hline 6 & Para rubber & 16.56 & 0.44 & 30.05 & 0.79 & 97.03 & 2.56 \\
\hline 7 & $\begin{array}{l}\text { Perennial trees and } \\
\text { orchards }\end{array}$ & 55.76 & 1.47 & 50.21 & 1.32 & 88.95 & 2.34 \\
\hline 8 & Forest land & 632 & 16.66 & 604.7 & 15.94 & 481.3 & 12.68 \\
\hline 9 & Waterbodies & 36.81 & 0.97 & 57.46 & 1.51 & 53.3 & 1.4 \\
\hline 10 & Rangeland & 26.03 & 0.69 & 72.11 & 1.9 & 71.65 & 1.89 \\
\hline 11 & Marshes and swamps & 11.64 & 0.31 & 33.4 & 0.88 & 27.73 & 0.73 \\
\hline 12 & Unused land & 28.57 & 0.75 & 34.33 & 0.9 & 93.32 & 2.46 \\
\hline & Total & 3794.22 & 100.00 & 3794.22 & 100.00 & 3794.22 & 100.00 \\
\hline
\end{tabular}

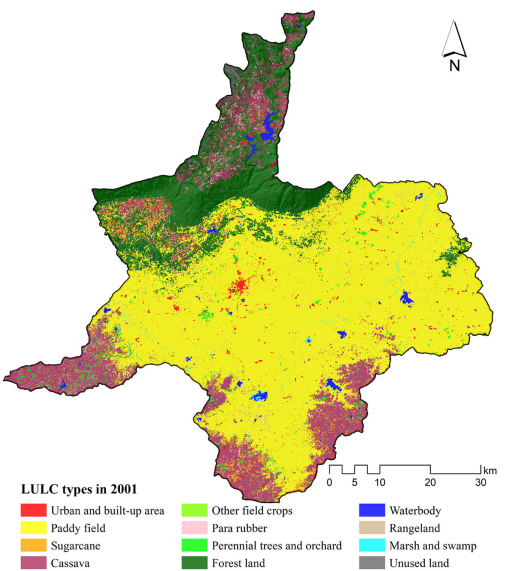

(a)

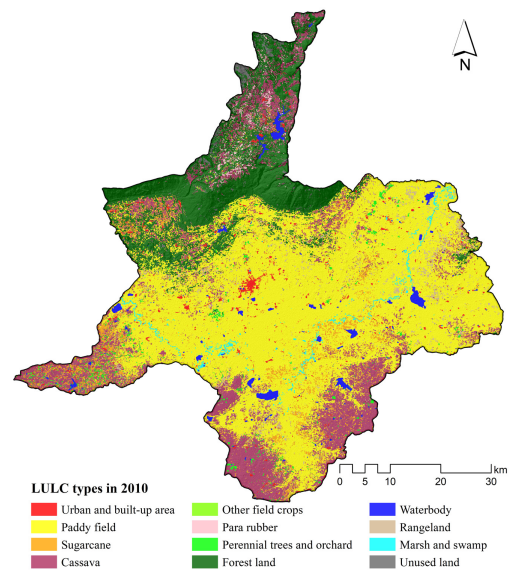

(b)

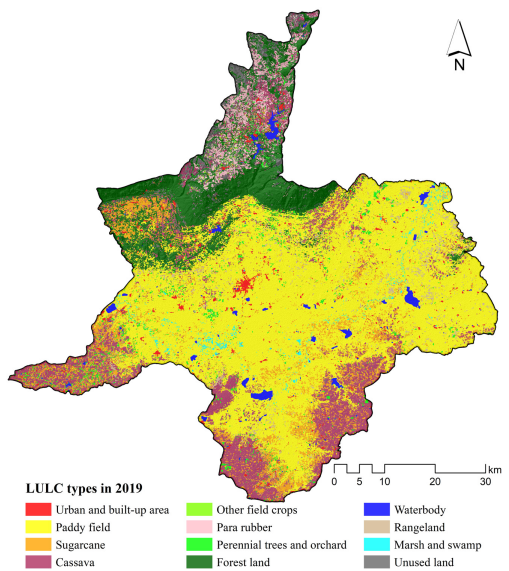

(c)

Figure 5. Spatial distribution of the LULC classification in: (a) 2001, (b) 2010, and (c) 2019.

The results reveal that in 2001, the top three most dominant LULC types were paddy fields $(61.79 \%)$, forest land $(16.66 \%)$, and cassava $(14.05 \%)$. On the contrary, the top three least dominant LULC types were other field crops $(0.06 \%)$, marshes and swamps $(0.30 \%)$, and para rubber $(0.44 \%)$, which are randomly distributed in the study area. Meanwhile, the top three most dominant in 2019 were paddy fields (54.58\%), cassava (16.59\%), and forest land $(15.94 \%)$. Conversely, the top three least dominant LULC types in 2001 were other field crops $(0.14 \%)$, marshes and swamps $(0.79 \%)$, and para rubber $(0.88 \%)$. Recently, the top three most dominant LULC types in 2019 were paddy fields (53.03\%), cassava (12.91\%), and forest land $(12.68 \%)$. In contrast, the top three least dominant LULC types were other field crops $(0.16 \%)$, marshes and swamps $(0.73 \%)$, and waterbodies $(1.40 \%)$. The temporal change in LULC type coverage in 2001, 2010, and 2019 is presented in Figure 6.

Furthermore, a simple comparison of the LULC type coverage with the changing area, annual change rate, and percentage of in two short periods (2001-2010 and 2010-2019) and in the long period (2001-2019) are reported in Tables 6-8. The annual change rate of the short- and long-term periods plays a significant role in land requirement estimation for LULC prediction using the CLUE-S model. 


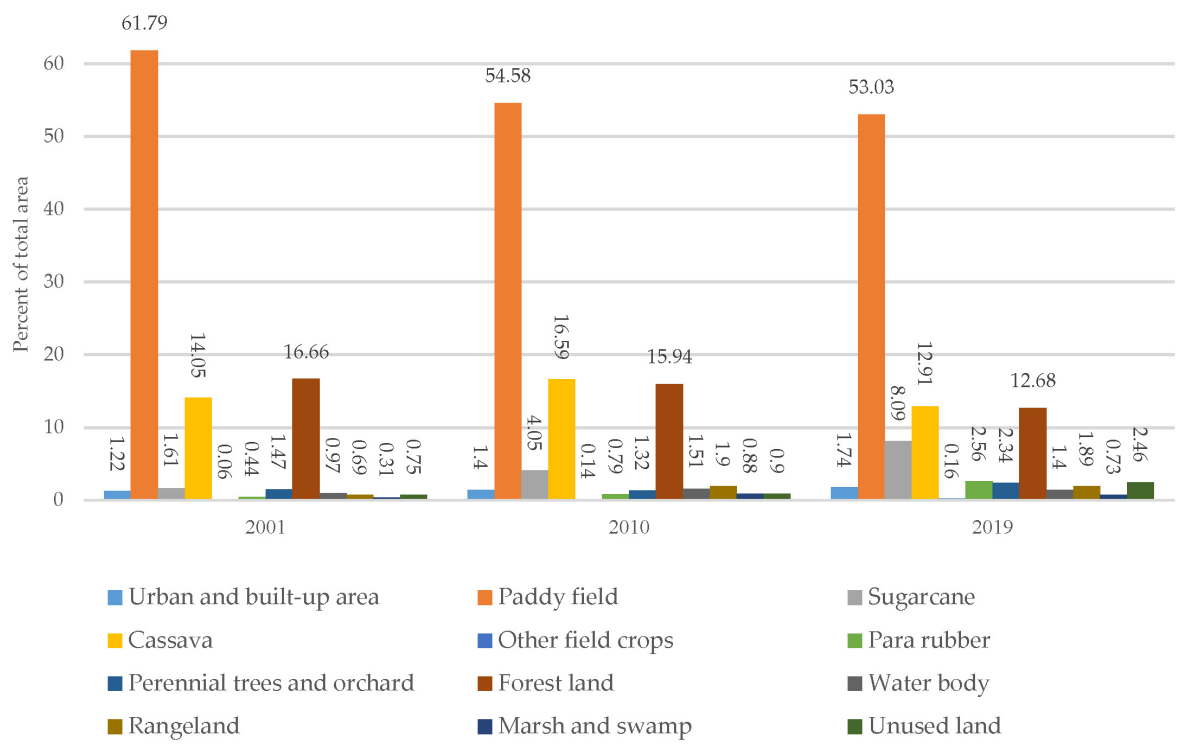

Figure 6. Multitemporal change of LULC type coverage in 2001, 2010, and 2019.

Table 6. Simple LULC change detection between 2001 and 2010.

\begin{tabular}{lcccccccccccc}
\hline \multirow{2}{*}{ LULC } & \multicolumn{10}{c}{ LULC Type (Area, $\mathbf{~ k m}^{\mathbf{2}}$ ) } \\
\cline { 2 - 13 } & UR & PA & SU & CA & PC & PR & PO & FO & WA & RA & MA & UL \\
\hline In 2001 & 46.17 & 2344.39 & 61.25 & 532.95 & 2.09 & 16.56 & 55.76 & 632.00 & 36.81 & 26.03 & 11.64 & 28.57 \\
In 2010 & 53.21 & 2070.71 & 153.52 & 629.33 & 5.19 & 30.05 & 50.21 & 604.70 & 57.46 & 72.11 & 33.40 & 34.33 \\
\hline Change area & $\mathbf{7 . 0 4}$ & $\mathbf{- 2 7 3 . 6 8}$ & $\mathbf{9 2 . 2 7}$ & $\mathbf{9 6 . 3 8}$ & $\mathbf{3 . 1 1}$ & $\mathbf{1 3 . 4 9}$ & $\mathbf{- 5 . 5 4}$ & $\mathbf{- 2 7 . 3 0}$ & $\mathbf{2 0 . 6 5}$ & $\mathbf{4 6 . 0 8}$ & $\mathbf{2 1 . 7 6}$ & $\mathbf{5 . 7 6}$ \\
\hline Annual change rate & $\mathbf{0 . 7 8}$ & $\mathbf{- 3 0 . 4 1}$ & $\mathbf{1 0 . 2 5}$ & $\mathbf{1 0 . 7 1}$ & $\mathbf{0 . 3 5}$ & $\mathbf{1 . 5 0}$ & $\mathbf{- 0 . 6 2}$ & $\mathbf{- 3 . 0 3}$ & $\mathbf{2 . 2 9}$ & $\mathbf{5 . 1 2}$ & $\mathbf{2 . 4 2}$ & $\mathbf{0 . 6 4}$ \\
\hline Percentage of change & $\mathbf{0 . 1 9}$ & $\mathbf{- 7 . 2 1}$ & $\mathbf{2 . 4 3}$ & $\mathbf{2 . 5 4}$ & $\mathbf{0 . 0 8}$ & $\mathbf{0 . 3 6}$ & $\mathbf{- 0 . 1 5}$ & $\mathbf{- 0 . 7 2}$ & $\mathbf{0 . 5 4}$ & $\mathbf{1 . 2 1}$ & $\mathbf{0 . 5 7}$ & $\mathbf{0 . 1 5}$ \\
\hline
\end{tabular}

Table 7. Simple LULC change detection between 2010 and 2019.

\begin{tabular}{lcccccccccccc}
\hline \multirow{2}{*}{ LULC } & \multicolumn{10}{c}{ LULC Type (Area, $\mathbf{k m}^{\mathbf{2}}$ ) } \\
\cline { 2 - 13 } & UR & PA & SU & CA & PC & PR & PO & FO & WA & RA & MA & UL \\
\hline In 2010 & 53.21 & 2070.71 & 153.52 & 629.33 & 5.19 & 30.05 & 50.21 & 604.70 & 57.46 & 72.11 & 33.40 & 34.33 \\
In 2019 & 65.84 & 2012.16 & 306.85 & 489.91 & 6.19 & 97.03 & 88.95 & 481.30 & 53.30 & 71.65 & 27.73 & 93.32 \\
\hline Change area & $\mathbf{1 2 . 6 3}$ & $\mathbf{- 5 8 . 5 5}$ & $\mathbf{1 5 3 . 3 3}$ & $\mathbf{- 1 3 9 . 4 1}$ & $\mathbf{1 . 0 0}$ & $\mathbf{6 6 . 9 9}$ & $\mathbf{3 8 . 7 3}$ & $\mathbf{- 1 2 3 . 4 1}$ & $\mathbf{- 4 . 1 6}$ & $\mathbf{- 0 . 4 7}$ & $\mathbf{- 5 . 6 7}$ & $\mathbf{5 8 . 9 9}$ \\
\hline Annual change rate & $\mathbf{1 . 4 0}$ & $\mathbf{- 6 . 5 1}$ & $\mathbf{1 7 . 0 4}$ & $\mathbf{- 1 5 . 4 9}$ & $\mathbf{0 . 1 1}$ & $\mathbf{7 . 4 4}$ & $\mathbf{4 . 3 0}$ & $\mathbf{- 1 3 . 7 1}$ & $\mathbf{- 0 . 4 6}$ & $\mathbf{- 0 . 0 5}$ & $\mathbf{- 0 . 6 3}$ & $\mathbf{6 . 5 5}$ \\
\hline Percentage of change & $\mathbf{0 . 3 3}$ & $\mathbf{- 1 . 5 4}$ & $\mathbf{4 . 0 4}$ & $\mathbf{- 3 . 6 7}$ & $\mathbf{0 . 0 3}$ & $\mathbf{1 . 7 7}$ & $\mathbf{1 . 0 2}$ & $\mathbf{- 3 . 2 5}$ & $\mathbf{- 0 . 1 1}$ & $\mathbf{- 0 . 0 1}$ & $\mathbf{- 0 . 1 5}$ & $\mathbf{1 . 5 5}$ \\
\hline
\end{tabular}

Table 8. Simple LULC change detection between 2001 and 2019.

\begin{tabular}{|c|c|c|c|c|c|c|c|c|c|c|c|c|}
\hline \multirow{2}{*}{ LULC } & \multicolumn{12}{|c|}{ LULC Type (Area, km²) } \\
\hline & UR & PA & SU & CA & PC & PR & PO & FO & WA & RA & MA & UL \\
\hline In 2001 & 46.17 & 2344.39 & 61.25 & 532.95 & 2.09 & 16.56 & 55.76 & 632 & 36.81 & 26.03 & 11.64 & 28.57 \\
\hline In 2019 & 65.84 & 2012.16 & 306.85 & 489.91 & 6.19 & 97.03 & 88.95 & 481.3 & 53.3 & 71.65 & 27.73 & 93.32 \\
\hline Change area & 19.67 & -332.23 & 245.6 & -43.04 & 4.1 & 80.47 & 33.19 & -150.7 & 16.49 & 45.62 & 16.09 & 64.75 \\
\hline Annual change rate & 1.09 & -18.46 & 13.64 & -2.39 & 0.23 & 4.47 & 1.84 & -8.37 & 0.92 & 2.53 & 0.89 & 3.60 \\
\hline Percentage of change & 0.52 & -8.76 & 6.47 & -1.13 & 0.11 & 2.12 & 0.87 & -3.97 & 0.43 & 1.20 & 0.42 & 1.71 \\
\hline
\end{tabular}


Moreover, by considering the performance of the RF classifier for LULC classification, the overall accuracy and Kappa hat coefficient of the classified LULC maps in 2001 were $89.88 \%$ and $84.88 \%$, in 2010 were $90.71 \%$ and $87.03 \%$, and in 2019 were $91.37 \%$ and $88.26 \%$. The overall accuracy of more than $85 \%$ of the three maps provides acceptable results, as suggested by [57]. Likewise, the Kappa hat coefficient of the three maps was more than $80 \%$, representing strong agreement or accuracy between the classified map and the ground reference information [58]. The derived overall accuracy and Kappa hat coefficient values of the classified LULC maps are comparable to those of other researchers who classified LULC maps based on Landsat data with the RF classifier [44,59-65]

\subsection{Driving Factor Identification for LULC Change}

The results of the binary logistics regression analysis for identifying LULC type location preference after the multicollinearity test are reported in Table 9. The most common vital driving factor for all LULC type changes was elevation. Meanwhile, the most important driving factors for field crops (sugarcane, cassava, and other field crops) included elevation, slope, and annual rainfall. The specific driving factors for each LULC type preference from binary logistics regression are further applied to allocate LULC type for predicting LULC change under the CLUE-S model.

Table 9. Multiple linear equations of each LULC type location preference and area under the curve value from logistic regression analysis.

\begin{tabular}{|c|c|c|c|c|c|c|c|c|c|c|c|c|}
\hline \multirow{2}{*}{$\begin{array}{l}\text { Driving } \\
\text { Factors }\end{array}$} & \multicolumn{12}{|c|}{ LULC Type } \\
\hline & UR & PD & SU & CA & FC & PR & OP & FO & WA & RA & MA & UL \\
\hline Constant & 0.0930 & 10.8831 & 94.6560 & -38.4766 & 207.9784 & 24.1870 & -38.4831 & 87.3484 & -4.6862 & -80.0118 & 5.0436 & -8.6344 \\
\hline & n. s. & -0.0947 & -0.0001 & 0.0042 & 0.0101 & 0.0083 & n. s. & 0.0032 & 0.0068 & -0.0043 & -0.0467 & 0.0093 \\
\hline$X_{2}$ & n. s. & n. s. & -0.1943 & -0.1030 & -0.2144 & -0.0730 & -0.1314 & 0.1886 & -0.6715 & n. s. & n. s. & -0.0290 \\
\hline $\mathrm{X}_{3}$ & n. s. & 0.0130 & -0.1264 & 0.0477 & -0.2850 & -0.0398 & 0.0454 & -0.1167 & n. s. & 0.0991 & n. s. & n. s. \\
\hline$X_{4}$ & n. s. & n. s. & n. s. & n. s. & n. s. & n. s. & n. s. & n. s. & n. s. & n. s. & n. s. & n. s. \\
\hline$X_{5}$ & 0.0008 & -0.0018 & -0.0117 & -0.0148 & n. s. & -0.0186 & -0.0043 & -0.0113 & n. s. & -0.0060 & -0.0059 & n. s. \\
\hline$X_{6}$ & -0.0064 & n. s. & n. s. & n. s. & n. s. & n. s. & n. s. & n. s. & 0.0014 & n. s. & n. s. & n. s. \\
\hline$x_{7}$ & n. s. & n. s. & n. s. & 0.0010 & n. s. & -0.0014 & n. s. & n. s. & -0.0037 & n. s. & -0.0053 & n. s. \\
\hline $\mathrm{X}_{8}$ & -0.0182 & n. s. & n. s. & n. s. & n. s. & n. s. & n. s. & n. s. & n. s. & n. s. & n. s. & n. s. \\
\hline AUC & 0.9824 & 0.9763 & 0.7677 & 0.7942 & 0.9355 & 0.9440 & 0.6186 & 0.9289 & 0.8318 & 0.7445 & 0.8628 & 0.8693 \\
\hline
\end{tabular}

Note: Elevation $\left(X_{1}\right)$, slope $\left(X_{2}\right)$, annual rainfall $\left(X_{3}\right)$, average income per capita at the sub-district level $\left(X_{4}\right)$, population density at the sub-district level $\left(X_{5}\right)$, distance to road network $\left(X_{6}\right)$, distance to stream $\left(X_{7}\right)$, and distance to the existing urban area $\left(X_{8}\right)$.

Moreover, the derived area under the curve (AUC) values for each LULC type allocation varied from 0.6185 to 0.9824 . This finding suggests a fair-to-excellent fit between the predicted and real LULC transition [66-68].

\subsection{Local Parameter for LULC Prediction Using the CLUE-S Model}

After a wall-to-wall accuracy assessment, by comparison, for the predicted LULC in 2019 with the classified LULC in 2019, the overall accuracy was 86.95\%, and the Kappa hat coefficient was $80.72 \%$. Thus, the significant driving factors by binomial logistic regression analysis were chosen as the optimal local driving factors on land use change for LULC prediction using the CLUE-S model in this study (see Table 9).

In the meantime, the optimal conversion matrix for each LULC type possibly changed between 2010 and 2019 (Table 10) and the land use type resistance (elasticity) values based on the transition probability matrix of LULC changed between 2010 and 2019 from the Markov Chain model (Table 11), identified as a local parameter for LULC prediction. Thus, the elasticity value of the urban and built-up areas, paddy fields, sugarcane, cassava, other field crops, para rubber, perennial trees and orchards, forest land, waterbodies, rangeland, marshes and swamps, and unused land were 1.00, 0.93, 0.93, 0.65, 0.99, 0.80, 0.99, 0.80, 0.91, $0.89,0.39$, and 0.96 , respectively. 
Table 10. Conversion matrix of possible changes between 2010 and 2019.

\begin{tabular}{|c|c|c|c|c|c|c|c|c|c|c|c|c|c|}
\hline & \multirow{2}{*}{ LULC Types } & \multicolumn{12}{|c|}{ LULC Type Possible Change in 2019} \\
\hline & & UR & PA & SU & CA & FC & PR & PO & FO & WA & RA & MA & UL \\
\hline \multirow{12}{*}{ 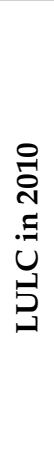 } & Urban and built-up areas (UR) & 1 & 0 & 0 & 0 & 0 & 0 & 0 & 0 & 0 & 0 & 0 & 0 \\
\hline & Paddy fields (PA) & 0 & 1 & 1 & 1 & 0 & 0 & 1 & 0 & 0 & 1 & 1 & 0 \\
\hline & Sugarcane (SU) & 1 & 1 & 1 & 1 & 0 & 0 & 1 & 0 & 0 & 0 & 0 & 0 \\
\hline & Cassava (CA) & 1 & 1 & 1 & 1 & 0 & 1 & 1 & 0 & 0 & 1 & 0 & 1 \\
\hline & Other field crops (FC) & 0 & 0 & 1 & 1 & 1 & 0 & 0 & 0 & 0 & 0 & 0 & 0 \\
\hline & Para rubber (PR) & 0 & 0 & 0 & 1 & 1 & 1 & 0 & 0 & 0 & 0 & 0 & 1 \\
\hline & Perennial trees and orchards (PO) & 0 & 0 & 1 & 0 & 0 & 0 & 1 & 0 & 0 & 0 & 0 & 0 \\
\hline & Forest land (FO) & 1 & 0 & 0 & 0 & 0 & 1 & 1 & 1 & 0 & 1 & 0 & 1 \\
\hline & Waterbodies (WA) & 1 & 0 & 1 & 0 & 0 & 0 & 0 & 0 & 1 & 0 & 1 & 0 \\
\hline & Rangeland (RA) & 0 & 1 & 0 & 0 & 0 & 0 & 1 & 0 & 0 & 1 & 0 & 0 \\
\hline & Marshes and swamps (MA) & 1 & 1 & 1 & 1 & 0 & 0 & 1 & 0 & 0 & 0 & 1 & 0 \\
\hline & Unused land (UL) & 1 & 1 & 0 & 0 & 0 & 0 & 0 & 0 & 0 & 0 & 0 & 1 \\
\hline
\end{tabular}

Table 11. Elasticity of LULC change for LULC prediction between 2010 and 2019.

\begin{tabular}{|c|c|c|c|c|c|c|c|c|c|c|c|c|c|}
\hline & \multirow{2}{*}{ LULC Types } & \multicolumn{12}{|c|}{ LULC Type Possible Change in 2019} \\
\hline & & UR & PA & SU & CA & FC & PR & PO & FO & WA & RA & MA & UL \\
\hline \multirow{12}{*}{ 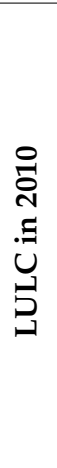 } & Urban and built-up areas (UR) & 1.00 & - & - & - & - & - & - & - & - & - & - & - \\
\hline & Paddy fields (PA) & - & 0.93 & 0.03 & 0.02 & - & - & 0.01 & - & - & - & 0.01 & - \\
\hline & Sugarcane (SU) & 0.01 & 0.01 & 0.93 & 0.05 & - & - & 0.01 & - & - & - & - & - \\
\hline & Cassava (CA) & 0.01 & 0.06 & 0.16 & 0.65 & - & 0.07 & 0.01 & - & - & - & - & 0.04 \\
\hline & Other field crops (FC) & - & - & - & - & 0.99 & - & 0.01 & - & - & - & - & - \\
\hline & Para rubber (PR) & - & - & - & 0.11 & 0.01 & 0.80 & 0.03 & - & - & - & - & 0.05 \\
\hline & Perennial trees and orchards (PO) & - & - & 0.01 & 0.01 & - & - & 0.99 & - & - & - & - & - \\
\hline & Forest land (FO) & - & 0.02 & - & 0.05 & - & 0.05 & 0.01 & 0.80 & - & - & - & 0.06 \\
\hline & Waterbodies (WA) & - & 0.04 & 0.02 & 0.01 & - & - & - & - & 0.91 & - & 0.01 & - \\
\hline & Rangeland (RA) & - & 0.08 & - & 0.02 & - & - & 0.01 & - & - & 0.89 & - & - \\
\hline & Marshes and swamps (MA) & 0.01 & 0.55 & 0.03 & - & - & - & 0.02 & - & - & - & 0.39 & - \\
\hline & Unused land (UL) & 0.01 & 0.03 & - & - & - & - & - & - & - & - & - & 0.96 \\
\hline
\end{tabular}

\subsection{LULC Prediction between 2002 and 2009}

LULC prediction data between 2002 and 2009, which were simultaneously allocated based on the conversion matrix of LULC change (Table 10), elasticity value (Table 11), annual land demand (Table 12), and driving factors on LULC change for specific LULC type location preference (Table 9), are reported in Table 13 and displayed in Figure 7.

Table 12. Annual land requirement of each LULC type between 2001 and 2010.

\begin{tabular}{|c|c|c|c|c|c|c|c|c|c|c|c|c|c|}
\hline \multirow{2}{*}{ Year } & \multicolumn{12}{|c|}{ LULC Type (Area, km²) } & \multirow{2}{*}{ Total } \\
\hline & UR & PA & SU & CA & FC & PR & PO & FO & WA & $\mathbf{R A}$ & MA & $\mathbf{U L}$ & \\
\hline 2001 & 46.17 & 2344.39 & 61.25 & 532.95 & 2.09 & 16.56 & 55.76 & 632.00 & 36.81 & 26.03 & 11.64 & 28.57 & 3794.22 \\
\hline 2002 & 46.95 & 2314.01 & 71.53 & 543.60 & 2.45 & 18.03 & 55.15 & 628.98 & 39.12 & 31.16 & 14.03 & 29.19 & 3794.22 \\
\hline 2003 & 47.71 & 2283.59 & 81.77 & 554.34 & 2.79 & 19.54 & 54.54 & 625.95 & 41.40 & 36.28 & 16.46 & 29.84 & 3794.22 \\
\hline 2004 & 48.50 & 2253.18 & 91.96 & 565.05 & 3.13 & 21.09 & 53.90 & 622.90 & 43.70 & 41.38 & 18.94 & 30.47 & 3794.22 \\
\hline 2005 & 49.30 & 2222.70 & 102.27 & 575.78 & 3.50 & 22.54 & 53.27 & 619.88 & 46.00 & 46.51 & 21.30 & 31.16 & 3794.22 \\
\hline 2006 & 50.05 & 2192.30 & 112.51 & 586.50 & 3.83 & 24.10 & 52.66 & 616.83 & 48.28 & 51.63 & 23.78 & 31.74 & 3794.22 \\
\hline 2007 & 50.82 & 2161.94 & 122.76 & 597.16 & 4.17 & 25.57 & 52.07 & 613.81 & 50.57 & 56.77 & 26.17 & 32.40 & 3794.22 \\
\hline 2008 & 51.61 & 2131.48 & 133.03 & 607.87 & 4.51 & 27.08 & 51.47 & 610.77 & 52.86 & 61.90 & 28.60 & 33.03 & 3794.22 \\
\hline 2009 & 52.41 & 2101.12 & 143.27 & 618.63 & 4.87 & 28.53 & 50.84 & 607.74 & 55.16 & 66.99 & 30.97 & 33.69 & 3794.22 \\
\hline 2010 & 53.21 & 2070.70 & 153.51 & 629.33 & 5.19 & 30.05 & 50.21 & 604.70 & 57.46 & 72.11 & 33.40 & 34.33 & 3794.22 \\
\hline $\begin{array}{c}\text { Annual } \\
\text { rate }\end{array}$ & 0.79 & $\begin{array}{c}- \\
30.42\end{array}$ & 10.24 & 10.71 & 0.33 & 1.52 & -0.63 & -3.03 & 2.29 & 5.12 & 2.44 & 0.64 & \\
\hline
\end{tabular}


Table 13. Area of predicted LULC type between 2002 and 2009.

\begin{tabular}{|c|c|c|c|c|c|c|c|c|}
\hline \multirow{2}{*}{ LULC Types } & \multicolumn{8}{|c|}{ Area of Predicted LULC Type $\left(\mathrm{km}^{2}\right)$} \\
\hline & 2002 & 2003 & 2004 & 2005 & 2006 & 2007 & 2008 & 2009 \\
\hline Urban and built-up areas (UR) & 46.95 & 47.71 & 48.5 & 49.3 & 50.05 & 50.82 & 51.61 & 52.41 \\
\hline Paddy fields (PD) & 2314.01 & 2283.59 & 2253.18 & 2222.70 & 2192.30 & 2161.94 & 2131.48 & 2101.12 \\
\hline Sugarcane (SU) & 71.53 & 81.77 & 91.96 & 102.27 & 112.51 & 122.76 & 133.03 & 143.27 \\
\hline Cassava (CA) & 543.6 & 554.34 & 565.05 & 575.78 & 586.5 & 597.16 & 607.87 & 618.63 \\
\hline Other field crops (FC) & 2.45 & 2.79 & 3.13 & 3.5 & 3.83 & 4.17 & 4.51 & 4.87 \\
\hline Para rubber $(\mathrm{PR})$ & 18.03 & 19.54 & 21.09 & 22.54 & 24.1 & 25.57 & 27.08 & 28.53 \\
\hline Perennial trees and orchards (PO) & 55.15 & 54.54 & 53.9 & 53.27 & 52.66 & 52.07 & 51.47 & 50.84 \\
\hline Forest land (FO) & 628.98 & 625.95 & 622.9 & 619.88 & 616.83 & 613.81 & 610.77 & 607.74 \\
\hline Waterbodies (WA) & 39.12 & 41.4 & 43.7 & 46 & 48.28 & 50.57 & 52.86 & 55.16 \\
\hline Rangeland (RA) & 31.16 & 36.28 & 41.38 & 46.51 & 51.63 & 56.77 & 61.9 & 66.99 \\
\hline Marshes and swamps (MA) & 14.03 & 16.46 & 18.94 & 21.3 & 23.78 & 26.17 & 28.6 & 30.97 \\
\hline Unused land (UL) & 29.19 & 29.84 & 30.47 & 31.16 & 31.74 & 32.4 & 33.03 & 33.69 \\
\hline Total & 3794.22 & 3794.22 & 3794.22 & 3794.22 & 3794.22 & 3794.22 & 3794.22 & 3794.22 \\
\hline
\end{tabular}
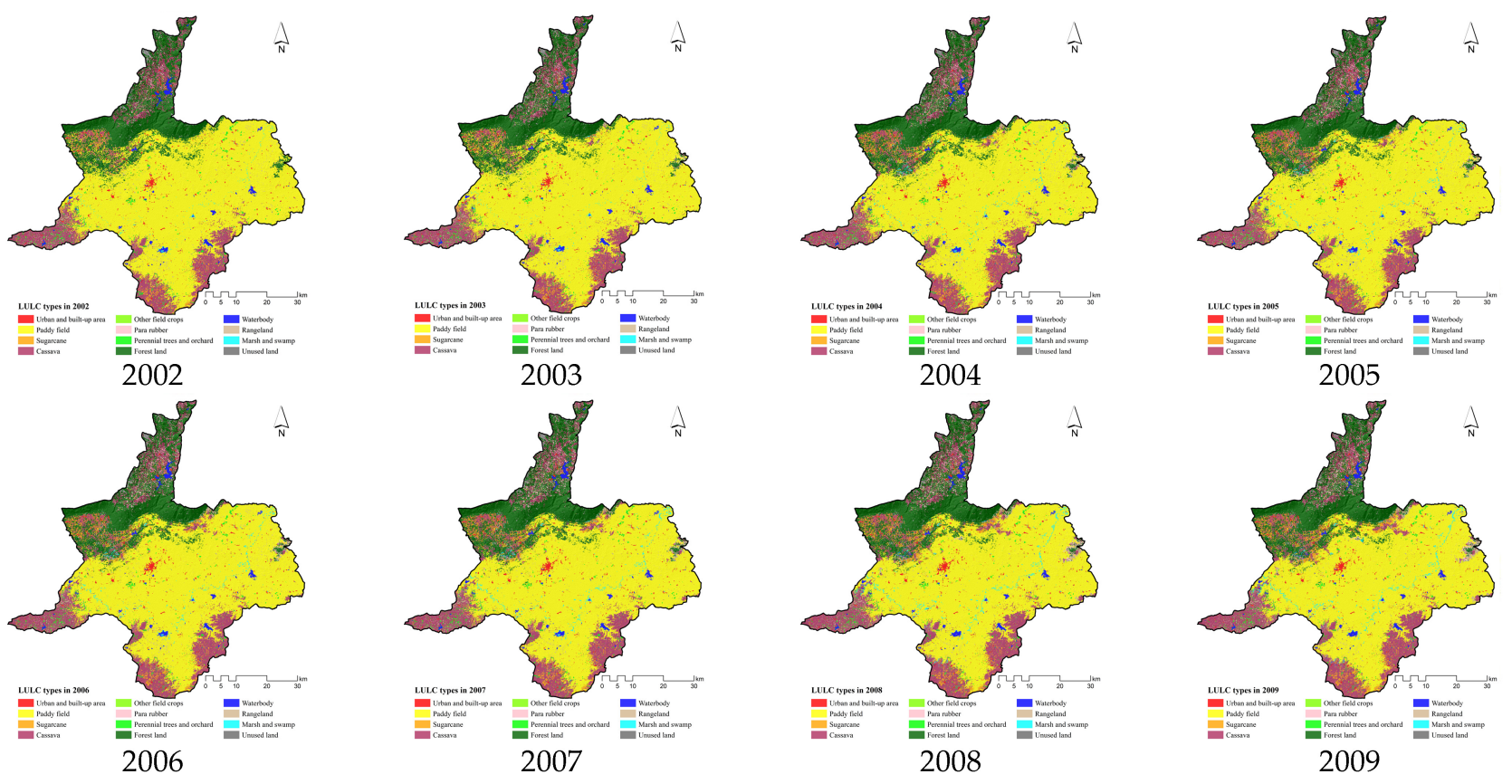

Figure 7. Spatial distribution of predicted LULC data between 2002 and 2009.

As a result, the LULC prediction between 2002 and 2009 was dictated by the historical LULC development between 2001 and 2010. The results indicate that the most increasing LULC types were cassava and sugarcane, with an increasing annual change rate of 10.71 and $10.24 \mathrm{~km}^{2}$ per year, while the paddy field was the most decreasing LULC type with a decreasing annual change rate of $30.42 \mathrm{~km}^{2}$ per year.

\subsection{LULC Prediction between 2011 and 2018}

The derived optimum local parameter of the CLUE-S model with annual land demand between 2010 and 2019 based on the annual change rate of the Markov Chain model (Table 14) was simultaneously combined with driving factors for LULC change for a specific LULC type location preference (Table 9) to predict LULC data, as shown by the results in Table 15 and Figure 8. 
Table 14. Annual land requirement of each LULC type between 2010 and 2019.

\begin{tabular}{|c|c|c|c|c|c|c|c|c|c|c|c|c|c|}
\hline \multirow{2}{*}{ Year } & \multicolumn{12}{|c|}{ LULC Type (Area, $\mathrm{km}^{2}$ ) } & \multirow{2}{*}{ Total } \\
\hline & UR & PA & SU & CA & FC & PR & PO & FO & WA & RA & MA & UL & \\
\hline 2010 & 53.21 & 2070.71 & 153.52 & 629.33 & 5.19 & 30.05 & 50.21 & 604.70 & 57.46 & 72.11 & 33.40 & 34.33 & 3794.22 \\
\hline 2011 & 54.58 & 2064.15 & 170.56 & 613.86 & 5.32 & 37.51 & 54.49 & 591.02 & 57.00 & 72.09 & 32.75 & 40.88 & 3794.22 \\
\hline 2012 & 55.99 & 2057.68 & 187.61 & 598.35 & 5.43 & 44.93 & 58.80 & 577.30 & 56.54 & 72.03 & 32.13 & 47.42 & 3794.22 \\
\hline 2013 & 57.46 & 2051.17 & 204.62 & 582.86 & 5.53 & 52.39 & 63.17 & 563.53 & 56.06 & 71.98 & 31.48 & 53.98 & 3794.22 \\
\hline 2014 & 58.81 & 2044.70 & 221.65 & 567.39 & 5.65 & 59.83 & 67.42 & 549.87 & 55.61 & 71.85 & 30.90 & 60.55 & 3794.22 \\
\hline 2015 & 60.27 & 2038.20 & 238.68 & 551.89 & 5.74 & 67.26 & 71.78 & 536.14 & 55.15 & 71.81 & 30.21 & 67.07 & 3794.22 \\
\hline 2016 & 61.65 & 2031.64 & 255.75 & 536.39 & 5.87 & 74.71 & 76.06 & 522.43 & 54.70 & 71.81 & 29.59 & 73.61 & 3794.22 \\
\hline 2017 & 63.06 & 2025.15 & 272.80 & 520.90 & 5.97 & 82.14 & 80.37 & 508.74 & 54.25 & 71.71 & 28.95 & 80.18 & 3794.22 \\
\hline 2018 & 64.41 & 2018.69 & 289.83 & 505.42 & 6.08 & 89.59 & 84.63 & 495.02 & 53.76 & 71.71 & 28.33 & 86.75 & 3794.22 \\
\hline 2019 & 65.84 & 2012.16 & 306.85 & 489.91 & 6.19 & 97.03 & 88.95 & 481.30 & 53.30 & 71.65 & 27.73 & 93.32 & 3794.22 \\
\hline $\begin{array}{l}\text { Annual } \\
\text { rate }\end{array}$ & 1.40 & -6.51 & 17.04 & $\begin{array}{c}- \\
15.49\end{array}$ & 0.11 & 7.44 & 4.30 & $\begin{array}{c}- \\
13.71\end{array}$ & -0.46 & 0.05 & -0.63 & 6.55 & \\
\hline
\end{tabular}

Table 15. Area of predicted LULC type between 2011 and 2018.

\begin{tabular}{|c|c|c|c|c|c|c|c|c|}
\hline \multirow{2}{*}{ LULC Types } & \multicolumn{8}{|c|}{ Area of Predicted LULC Type $\left(\mathrm{km}^{2}\right)$} \\
\hline & 2011 & 2012 & 2013 & 2014 & 2015 & 2016 & 2017 & 2018 \\
\hline Urban and built-up areas (UR) & 54.58 & 55.99 & 57.46 & 58.81 & 60.27 & 61.65 & 63.06 & 64.41 \\
\hline Paddy fields (PD) & 2064.15 & 2057.68 & 2051.17 & 2044.70 & 2038.20 & 2031.64 & 2025.15 & 2018.69 \\
\hline Sugarcane (SU) & 170.56 & 187.61 & 204.62 & 221.65 & 238.68 & 255.75 & 272.8 & 289.83 \\
\hline Cassava (CA) & 613.86 & 598.35 & 582.86 & 567.39 & 551.89 & 536.39 & 520.9 & 505.42 \\
\hline Other field crops (FC) & 5.32 & 5.43 & 5.53 & 5.65 & 5.74 & 5.87 & 5.97 & 6.08 \\
\hline Para rubber (PR) & 37.51 & 44.93 & 52.39 & 59.83 & 67.26 & 74.71 & 82.14 & 89.59 \\
\hline Perennial trees and orchards (PO) & 54.49 & 58.8 & 63.17 & 67.42 & 71.78 & 76.06 & 80.37 & 84.63 \\
\hline Forest land (FO) & 591.02 & 577.3 & 563.53 & 549.87 & 536.14 & 522.43 & 508.74 & 495.02 \\
\hline Waterbodies (WA) & 57 & 56.54 & 56.06 & 55.61 & 55.15 & 54.7 & 54.25 & 53.76 \\
\hline Rangeland (RA) & 72.09 & 72.03 & 71.98 & 71.85 & 71.81 & 71.81 & 71.71 & 71.71 \\
\hline Marshes and swamps (MA) & 32.75 & 32.13 & 31.48 & 30.9 & 30.21 & 29.59 & 28.95 & 28.33 \\
\hline Unused land (UL) & 40.88 & 47.42 & 53.98 & 60.55 & 67.07 & 73.61 & 80.18 & 86.75 \\
\hline Total & 3794.22 & 3794.22 & 3794.22 & 3794.22 & 3794.22 & 3794.22 & 3794.22 & 3794.22 \\
\hline
\end{tabular}
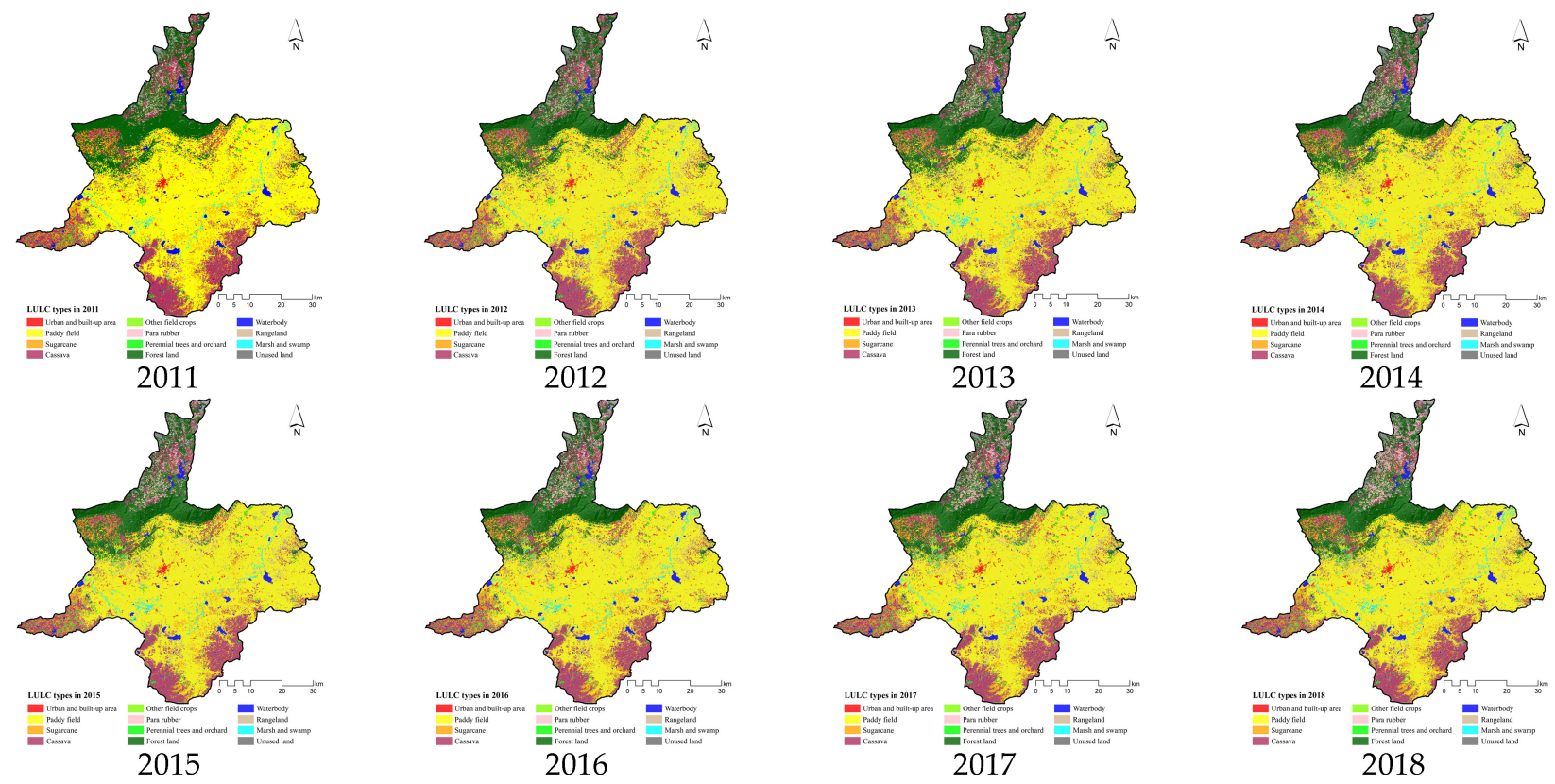

Figure 8. Spatial distribution of the predicted LULC data between 2011 and 2018. 
As with the previous results, the LULC prediction between 2011 and 2018 was enforced by the historical LULC development between 2010 and 2019. Again, the results revealed that sugarcane was the most increasing LULC type, with an increasing annual rate of $17.04 \mathrm{~km}^{2}$ per year, while cassava and forest land were the most decreasing LULC types, with decreasing annual change rates of 15.49 and $13.71 \mathrm{~km}^{2}$ per year.

In summary, the proportional area of the time series LULC data between 2001 and 2019 based on RF classification and CLUE-S prediction is displayed in Figure 9.

Time-series LULC data between 2001 and 2019

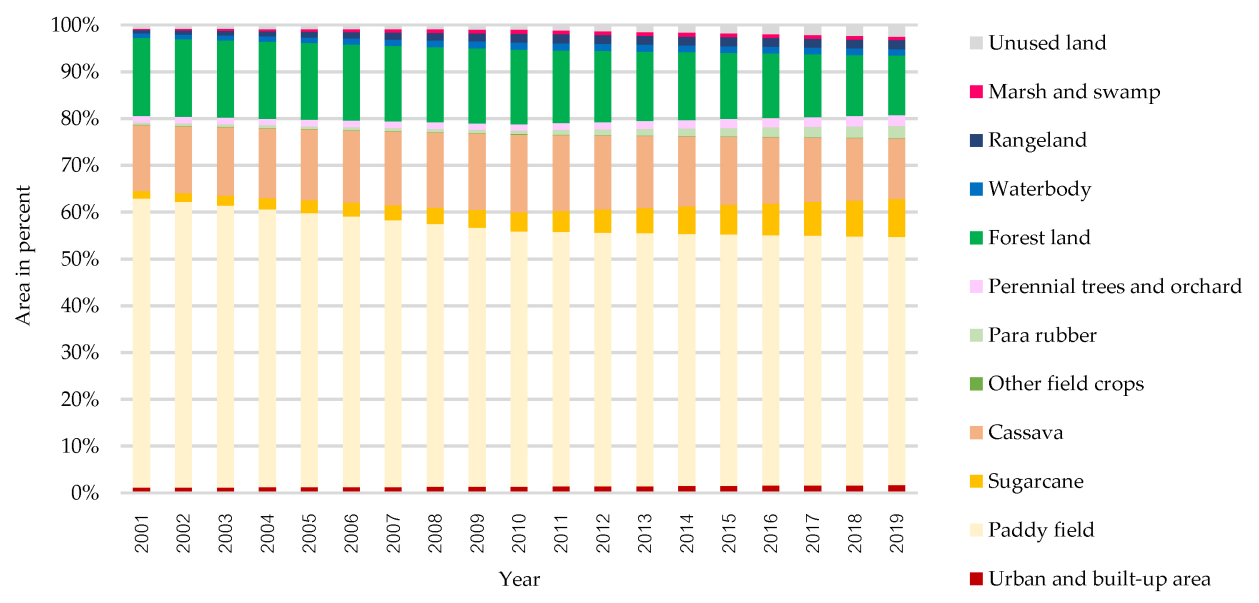

Figure 9. Proportion area of LULC type of LULC data between 2001 and 2019.

\subsection{Surface Runoff Estimation between 2001 and 2010}

The surface runoff estimation between 2001 and 2010 was estimated with three runoff curve number $(\mathrm{CN})$ values assigned based on three different AMC conditions (see Table 3) for suitable AMC identification in the study area. In principle, the AMC is an indicator of watershed wetness and availability of soil storage. The annual surface runoff volume of the three AMC conditions with rainfall data between 2001 and 2010 is presented in Table 16 and Figure 10.

Table 16. Annual surface runoff volume of the three AMC conditions and rainfall data between 2001 and 2010.

\begin{tabular}{|c|c|c|c|c|}
\hline \multirow{2}{*}{ Year } & \multicolumn{3}{|c|}{ Surface Runoff Volume (million $\mathrm{m}^{3}$ ) } & \multirow{2}{*}{$\begin{array}{l}\text { Annual Rainfall * } \\
(\mathrm{mm})\end{array}$} \\
\hline & AMC-I & AMC-II & AMC-III & \\
\hline 2001 & 1178.41 & 939.70 & 1537.88 & 939.70 \\
\hline 2002 & 4057.74 & 1191.60 & 4652.40 & 1191.60 \\
\hline 2003 & 1444.46 & 900.80 & 1836.21 & 900.80 \\
\hline 2004 & 1335.34 & 915.40 & 1716.51 & 915.40 \\
\hline 2005 & 1754.45 & 1039.00 & 2185.22 & 1039.00 \\
\hline 2006 & 4229.13 & 1196.00 & 4847.85 & 1196.00 \\
\hline 2007 & 3234.72 & 1342.90 & 3795.55 & 1342.90 \\
\hline 2008 & 5869.54 & 1695.20 & 6558.70 & 1695.20 \\
\hline 2009 & 4189.38 & 1502.10 & 4804.03 & 1502.10 \\
\hline 2010 & 5761.81 & 1506.30 & 6437.31 & 1506.30 \\
\hline
\end{tabular}

* Annual rainfall data at Chaiyaphum meteorological station, located at the center of the study area.

According to the results, the accumulated surface runoff volume under three AMC conditions was relatively different, because the $\mathrm{CN}$ values of these conditions were different (Table 3), while annual rainfall data were the same. The patterns of surface runoff volume under the three AMC conditions and annual rainfall between 2001 and 2010 were similar. The higher the annual rainfall, the higher the surface runoff. This finding was confirmed by simple linear regression analysis, as shown in Figure 11. The surface runoff volume under the three AMC conditions positively correlated with annual rainfall, with $\mathrm{R}^{2}$ values of 
$0.8503,0.8511$, and 0.8513 , respectively. These coefficient values show a strong relationship between annual rainfall and surface runoff, according to [50].

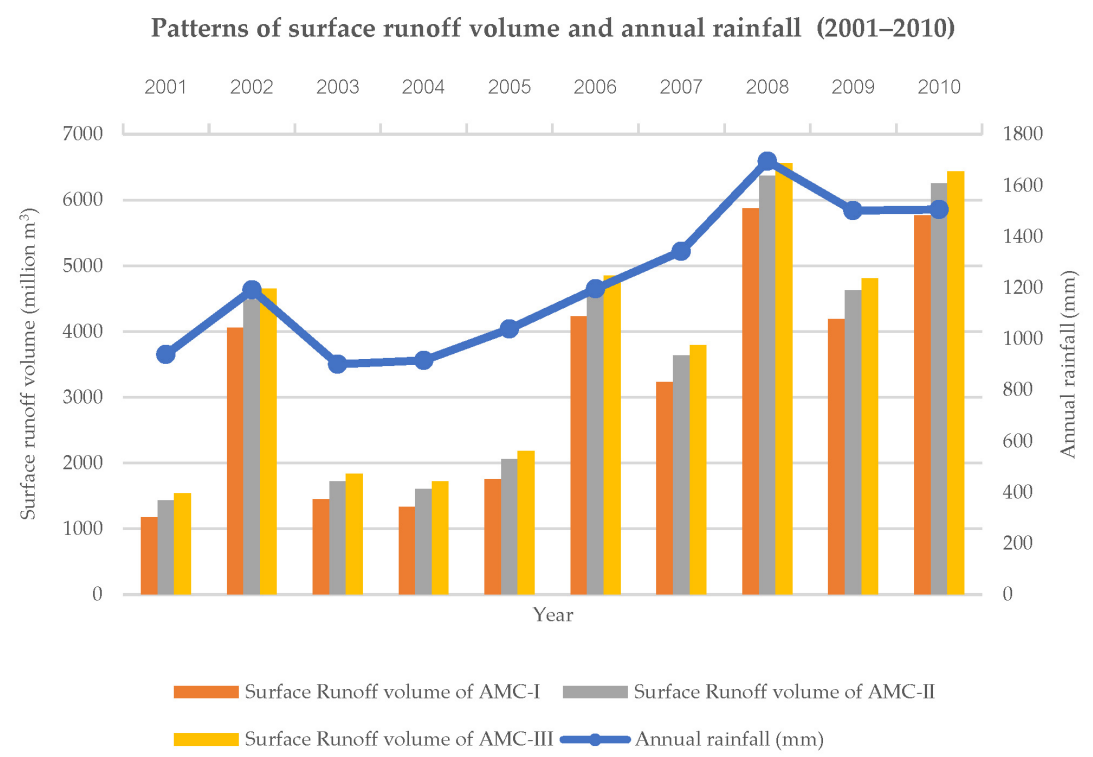

Figure 10. Patterns of surface runoff volume of the three AMC conditions and annual rainfall data between 2001 and 2010.

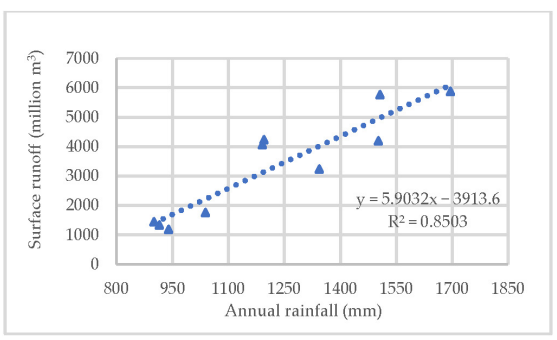

(a)

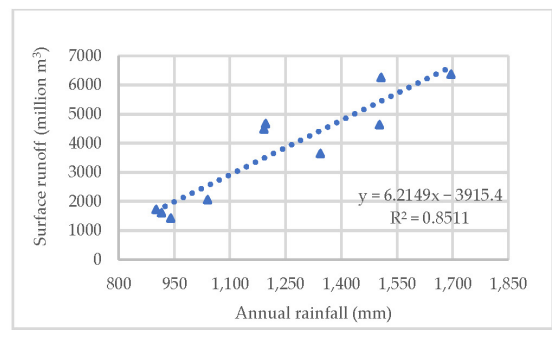

(b)

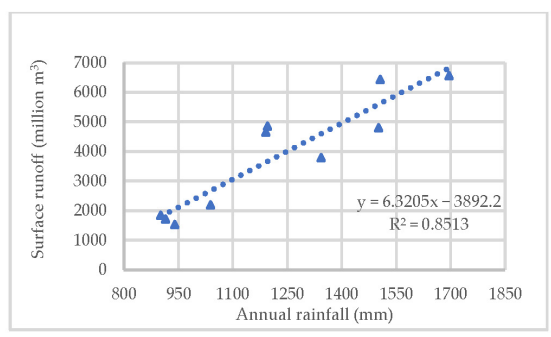

(c)

Figure 11. Relationship between surface runoff volume and annual rainfall: (a) AMC-I, (b) AMC-II, and (c) AMC-III.

In addition, the results of model performance using NSE, $\mathrm{R}^{2}$, and PBIAS for identifying the suitable AMC for surface runoff estimation based on observed and simulated data between 2001 and 2010 (Table 17) are reported in Table 18.

Table 17. Annual surface runoff volume of the three AMC conditions and rainfall data between 2001 and 2010.

\begin{tabular}{|c|c|c|c|c|c|c|c|c|c|c|c|c|}
\hline \multirow{3}{*}{ Year } & \multicolumn{4}{|c|}{ E.21 Hydrological Station } & \multicolumn{4}{|c|}{ E.23 Hydrological Station } & \multicolumn{4}{|c|}{ E.6C Hydrological Station } \\
\hline & \multirow{2}{*}{$Q_{o b s}$} & AMC-I & AMC-II & AMC-III & \multirow{2}{*}{$Q_{o b s}$} & AMC-I & AMC-II & AMC-III & \multirow{2}{*}{$Q_{o b s}$} & AMC-I & AMC-II & AMC-III \\
\hline & & $Q_{\text {sim }}$ & $Q_{\text {sim }}$ & $Q_{\text {sim }}$ & & $Q_{\text {sim }}$ & $Q_{\text {sim }}$ & $Q_{\text {sim }}$ & & $Q_{\text {sim }}$ & $Q_{\text {sim }}$ & $Q_{\text {sim }}$ \\
\hline 2001 & 633.4 & 601.82 & 695.9 & 725.99 & 373.1 & 292.79 & 354.69 & 382.87 & 36.64 & 25.82 & 45.19 & 61.25 \\
\hline 2002 & 1954.20 & 1820.05 & 1959.27 & 2001.76 & 1500.70 & 929.33 & 1032.16 & 1074.94 & 166.38 & 113.11 & 169.23 & 204.97 \\
\hline 2003 & 656.7 & 650.03 & 746.73 & 777.44 & 575.6 & 316.3 & 379.93 & 408.74 & 50.63 & 21.6 & 37.64 & 51.78 \\
\hline 2004 & 549.9 & 838.14 & 945 & 978.53 & 542.4 & 409.53 & 482.1 & 514.13 & 41.21 & 31.16 & 53.41 & 71.19 \\
\hline 2005 & 854.7 & 1077.71 & 1195.42 & 1232.06 & 653.8 & 530 & 612.45 & 648.2 & 84.33 & 54.38 & 90.48 & 116.27 \\
\hline 2006 & 2230.50 & 2439.22 & 2590.81 & 2636.54 & 1630.70 & 1246.65 & 1364.56 & 1412.19 & 73.37 & 35.22 & 61.22 & 81.35 \\
\hline 2007 & 1914.30 & 1450.86 & 1581.28 & 1621.40 & 845.5 & 724.36 & 819.84 & 859.75 & 85.42 & 47.58 & 82.78 & 107.02 \\
\hline 2008 & 3494.50 & 3173.60 & 3334.97 & 3383.21 & 1932.20 & 1625.72 & 1754.19 & 1804.98 & 180.45 & 146.87 & 214.7 & 256.12 \\
\hline 2009 & 2180.20 & 2181.08 & 2328.33 & 2372.98 & 1560.00 & 1121.68 & 1234.52 & 1280.50 & 151.03 & 122.82 & 184.17 & 222.61 \\
\hline 2010 & 3188.90 & 3033.46 & 3189.97 & 3237.17 & 2174.40 & 1570.83 & 1698.55 & 1749.15 & 206.15 & 181.51 & 255.45 & 299.02 \\
\hline Average & 1765.73 & 1726.60 & 1856.77 & 1896.71 & 1178.84 & 876.72 & 973.3 & 1013.54 & 107.56 & 78.01 & 119.43 & 147.16 \\
\hline
\end{tabular}


Table 18. Statistical data of model performance for suitable AMC identification and model validation.

\begin{tabular}{|c|c|c|c|c|c|c|c|c|c|c|}
\hline \multirow{2}{*}{ AMC } & \multirow{2}{*}{ Year } & \multicolumn{3}{|c|}{ E.21 Station } & \multicolumn{3}{|c|}{ E.23 Station } & \multicolumn{3}{|c|}{ E.6C Station } \\
\hline & & NSE & $\mathbf{R}^{2}$ & PBIAS & NSE & $\mathbf{R}^{2}$ & PBIAS & NSE & $\mathbf{R}^{2}$ & PBIAS \\
\hline \multirow{11}{*}{ AMC-II } & 2001 & \multirow{11}{*}{0.95} & \multirow{11}{*}{0.96} & 0.18 & \multirow{11}{*}{0.67} & \multirow{11}{*}{0.96} & 0.68 & \multirow{10}{*}{0.71} & \multirow{11}{*}{0.96} & 1.01 \\
\hline & 2002 & & & 0.76 & & & 4.85 & & & 4.95 \\
\hline & 2003 & & & 0.04 & & & 2.20 & & & 2.70 \\
\hline & 2004 & & & -1.63 & & & 1.13 & & & 0.93 \\
\hline & 2005 & & & -1.26 & & & 1.05 & & & 2.78 \\
\hline & 2006 & & & -1.18 & & & 3.26 & & & 3.55 \\
\hline & 2007 & & & 2.62 & & & 1.03 & & & 3.52 \\
\hline & 2008 & & & 1.82 & & & 2.60 & & & 3.12 \\
\hline & 2009 & & & -0.01 & & & 3.72 & & & 2.62 \\
\hline & 2010 & & & 0.88 & & & 5.12 & & & 2.29 \\
\hline & 2001 & & & -0.35 & & & 0.16 & \multirow{10}{*}{0.85} & & -0.79 \\
\hline \multirow{8}{*}{ AMC-II } & 2002 & \multirow{9}{*}{0.94} & \multirow{8}{*}{0.96} & -0.03 & \multirow{8}{*}{0.82} & \multirow{8}{*}{0.96} & 3.97 & & \multirow{8}{*}{0.97} & -0.27 \\
\hline & 2003 & & & -0.51 & & & 1.66 & & & 1.21 \\
\hline & 2004 & & & -2.24 & & & 0.51 & & & -1.13 \\
\hline & 2005 & & & -1.93 & & & 0.35 & & & -0.57 \\
\hline & 2006 & & & -2.04 & & & 2.26 & & & 1.13 \\
\hline & 2007 & & & 1.89 & & & 0.22 & & & 0.25 \\
\hline & 2008 & & & 0.90 & & & 1.51 & & & -3.18 \\
\hline & 2009 & & & -0.84 & & & 2.76 & & & -3.08 \\
\hline \multirow{11}{*}{ AMC-III } & 2010 & & \multirow{11}{*}{0.96} & -0.01 & \multirow{11}{*}{0.86} & \multirow{11}{*}{0.96} & 4.04 & & \multirow{11}{*}{0.97} & -4.58 \\
\hline & 2001 & \multirow{10}{*}{0.94} & & -0.52 & & & -0.08 & \multirow{10}{*}{0.32} & & -2.29 \\
\hline & 2002 & & & -0.27 & & & 3.61 & & & -3.59 \\
\hline & 2003 & & & -0.68 & & & 1.42 & & & -0.11 \\
\hline & 2004 & & & -2.43 & & & 0.24 & & & -2.79 \\
\hline & 2005 & & & -2.14 & & & 0.05 & & & -2.97 \\
\hline & 2006 & & & -2.30 & & & 1.85 & & & -0.74 \\
\hline & 2007 & & & 1.66 & & & -0.12 & & & -2.01 \\
\hline & 2008 & & & 0.63 & & & 1.08 & & & -7.03 \\
\hline & 2009 & & & -1.09 & & & 2.37 & & & -6.66 \\
\hline & 2010 & & & -0.27 & & & 3.61 & & & -8.63 \\
\hline
\end{tabular}

As a result, in Table 18, under the AMC-I condition, the NSE value varied from 0.67 to 0.95 , while the $\mathrm{R}^{2}$ value varied from 0.71 to 0.96 (Figure 12). The PBIAS values ranged from $-1.63 \%$ for overestimation to $2.62 \%$ for underestimation. Meanwhile, under the AMC-II condition, the NSE value varied from 0.86 to 0.94 , while the $\mathrm{R}^{2}$ value varied from 0.96 to 0.97 (Figure 13). The PBIAS values ranged from $-4.58 \%$ for overestimation to $1.21 \%$ for underestimation. In the meantime, under the AMC-III condition, the NSE value varied from 0.32 to 0.96 , while the $\mathrm{R}^{2}$ value varies from 0.96 to 0.97 (Figure 14). The PBIAS values ranged from $-8.63 \%$ to $-0.11 \%$ for overestimation.

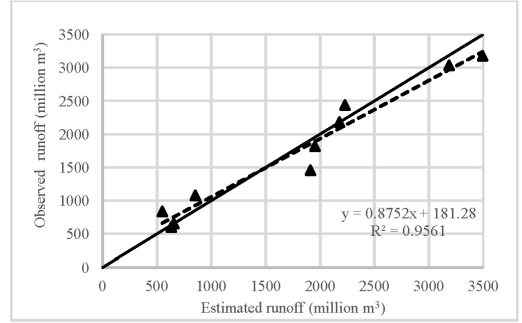

(a)

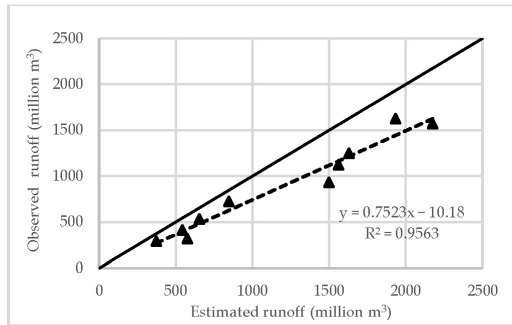

(b)

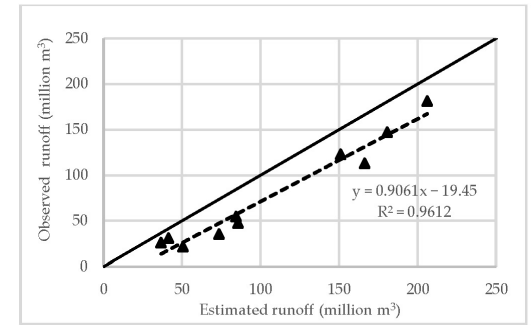

(c)

Figure 12. Relationship between the observed and estimated runoff between 2001 and 2010 under the AMC-I condition at the three stations: (a) E.21 station, (b) E.23 station, and (c) E.6C station. 


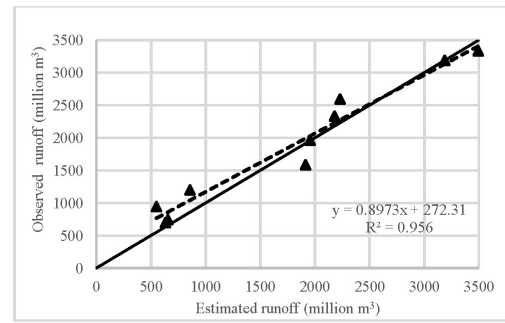

(a)

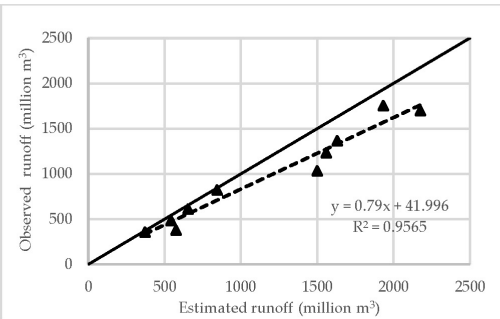

(b)

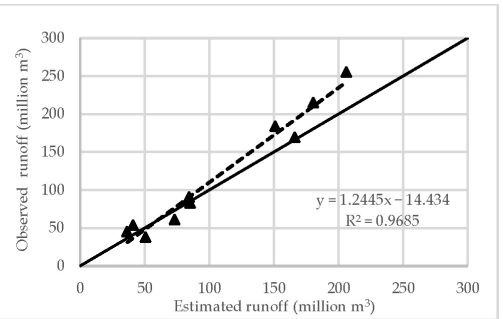

(c)

Figure 13. Relationship between the observed and estimated runoff between 2001 and 2010 under the AMC-II condition at the three stations: (a) E.21 station, (b) E.23 station, and (c) E.6C station.

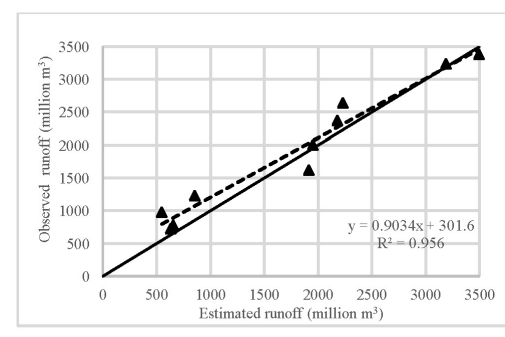

(a)

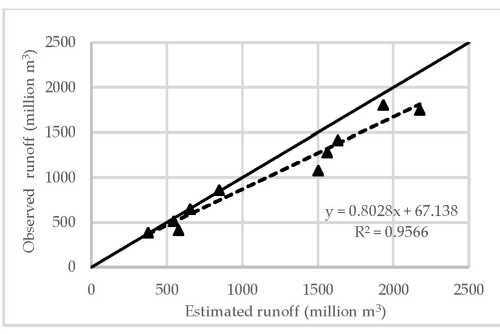

(b)

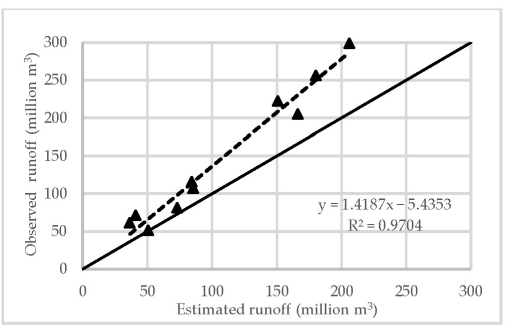

(c)

Figure 14. Relationship between the observed and estimated runoff between 2001 and 2010 under the AMC-III condition at the three stations: (a) E.21 station, (b) E.23 station, and (c) E.6C station.

These findings indicate that the NSE values under the three AMCs conditions can provide a perfect fit for surface runoff estimation. Likewise, the $\mathrm{R}^{2}$ values indicate a very high correlation between the observed and estimated surface runoff for all AMC conditions. Similarly, the PBIAS values show a relatively different overestimation and underestimation among three AMC conditions less than $\pm 10 \%$. These results show a very good fit between the observed and estimated surface runoff for the surface runoff estimation using the SCS-CN method, as suggested by [50] (see Table 3).

Furthermore, the NSE, $\mathrm{R}^{2}$, and PBIAS of all of the AMCs were compared to identify the suitable AMC for surface runoff estimation between 2011 and 2019, as shown in Table 19. As a result, AMC-II provided better overall average statistics measurements than the other AMCs. Thus, the AMC-II condition was chosen as the suitable AMC for surface runoff estimation in the second period (2011-2019).

Table 19. Comparison of the average statistics measurement for suitable AMC examination.

\begin{tabular}{cccc}
\hline \multirow{2}{*}{ AMC } & \multicolumn{3}{c}{ Average Statistics Measurement } \\
\cline { 2 - 4 } & NSE & $\mathbf{R}^{\mathbf{2}}$ & PBIAS \\
\hline AMC-I & 0.7767 & 0.9600 & 1.8443 \\
AMC-II & 0.8700 & 0.9633 & 0.0423 \\
AMC-III & 0.7067 & 0.9633 & -1.0067 \\
\hline
\end{tabular}

\subsection{Surface Runoff Estimation between 2011 and 2019}

The LULC and annual rainfall data between 2011 and 2019 as dynamic input with the hydrologic soil group were applied to estimate the surface runoff with the suitable AMC-II using the Model Builder of ArcGIS (See Figure 3). The annual surface runoff volume with rainfall data between 2011 and 2019 is reported in Table 20 and Figure 15. 
Table 20. Annual surface runoff volume and rainfall data between 2011 and 2019.

\begin{tabular}{ccc}
\hline Year & Surface Runoff Volume $\left(\mathbf{m i l l i o n} \mathbf{~ m}^{\mathbf{3}}\right)$ & Annual Rainfall $(\mathbf{m m})$ \\
\hline 2011 & 6142.43 & 1428.30 \\
2012 & 3583.03 & 1087.20 \\
2013 & 4200.48 & 1333.30 \\
2014 & 1003.60 & 793.50 \\
2015 & 1233.80 & 919.30 \\
2016 & 3475.16 & 1044.20 \\
2017 & 5433.33 & 1281.80 \\
2018 & 2588.57 & 809.00 \\
2019 & 1445.54 & 752.60 \\
\hline
\end{tabular}

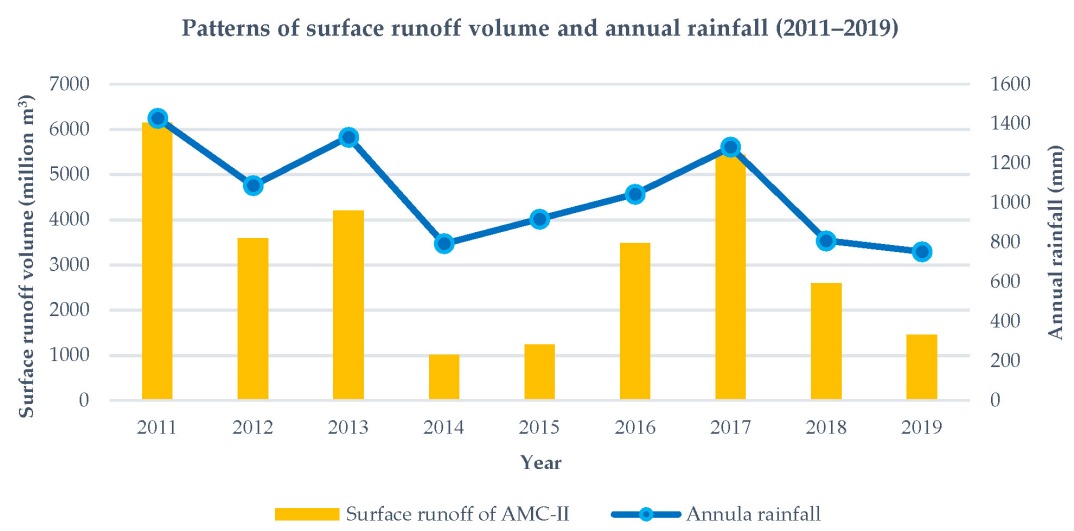

Figure 15. Pattern of surface runoff volume and annual rainfall of AMC-II between 2011 and 2019.

As a result, the surface runoff volume ranged from approximately 1004 million $\mathrm{m}^{3}$ in 2014 to approximately 6142 million $\mathrm{m}^{3}$ in 2011. The pattern of surface runoff volume and annual rainfall between 2011 and 2019 was similar. As mentioned in the previous section, the surface runoff volume positively correlated with annual rainfall, with an $R^{2}$ of 0.8511 . This finding is consistent with previous studies [69,70].

Furthermore, the results of model performance using NSE, $\mathrm{R}^{2}$, and PBIAS for validating surface runoff estimation based on observed and simulated data between 2011 and 2019 (Table 21) are reported in Table 22.

Table 21. Comparison of the observed $\left(Q_{o b s}\right)$ and simulated $\left(Q_{s i m}\right)$ surface runoff between 2011 and 2019 of the three stations.

\begin{tabular}{ccccccc}
\hline & \multicolumn{2}{c}{ E.21 Station } & \multicolumn{2}{c}{ E.23 Station } & \multicolumn{2}{c}{ E.63 Station } \\
\hline Year & $Q_{\text {obs }}$ & $Q_{\text {sim }}$ & $Q_{\text {obs }}$ & $Q_{\text {sim }}$ & $Q_{\text {obs }}$ & $Q_{\text {sim }}$ \\
\hline 2011 & 2943.40 & 2919.23 & 2283.30 & 1954.57 & 175.78 & 158.26 \\
2012 & 1989.10 & 1694.14 & 677.20 & 882.97 & 112.15 & 151.54 \\
2013 & 1884.80 & 2058.57 & 1646.70 & 1492.01 & 70.59 & 79.15 \\
2014 & 339.30 & 600.38 & 330.40 & 299.17 & 19.87 & 23.05 \\
2015 & 440.80 & 547.04 & 418.70 & 272.62 & 27.32 & 53.76 \\
2016 & 1563.30 & 1490.67 & 1058.30 & 771.66 & 87.26 & 75.27 \\
2017 & 2732.70 & 2668.97 & 1823.20 & 1563.06 & 182.55 & 166.29 \\
2018 & 923.60 & 1089.07 & 665.80 & 573.76 & 64.48 & 78.83 \\
2019 & 60.10 & 795.50 & 84.20 & 412.55 & 38.25 & 59.31 \\
\hline Average & $\mathbf{1 4 3 0 . 7 9}$ & $\mathbf{1 5 4 0 . 4 0}$ & $\mathbf{9 9 8 . 6 4}$ & $\mathbf{9 1 3 . 6 0}$ & $\mathbf{8 6 . 4 7}$ & $\mathbf{9 3 . 9 4}$ \\
\hline
\end{tabular}

Note: Observed $\left(Q_{o b s}\right)$ and simulated $\left(Q_{\text {sim }}\right)$ surface runoff in a million $\mathrm{m}^{3}$. 
Table 22. Statistical model performance data for the surface runoff estimation between 2011 and 2019 at the three stations.

\begin{tabular}{|c|c|c|c|c|c|c|c|c|c|}
\hline \multirow{2}{*}{ Year } & \multicolumn{3}{|c|}{ E.21 Station } & \multicolumn{3}{|c|}{ E.23 Station } & \multicolumn{3}{|c|}{ E.6C Station } \\
\hline & NSE & $\mathbf{R}^{2}$ & PBIAS & NSE & $\mathbf{R}^{2}$ & PBIAS & NSE & $\mathbf{R}^{2}$ & PBIAS \\
\hline 2011 & 0.91 & 0.94 & 0.19 & 0.90 & 0.94 & 3.66 & 0.87 & 0.90 & 2.25 \\
\hline 2012 & & & 2.29 & & & -2.29 & & & -5.06 \\
\hline 2013 & & & -1.35 & & & 1.72 & & & -1.10 \\
\hline 2014 & & & -2.03 & & & 0.35 & & & -0.41 \\
\hline 2015 & & & -0.83 & & & 1.63 & & & -3.40 \\
\hline 2016 & & & 0.56 & & & 3.19 & & & 1.54 \\
\hline 2017 & & & 0.49 & & & 2.89 & & & 2.09 \\
\hline 2018 & & & -1.28 & & & 1.02 & & & -1.84 \\
\hline 2019 & & & -5.71 & & & -3.65 & & & -2.71 \\
\hline
\end{tabular}

As a result, in Table 22, the NSE value from three stations varied from 0.87 to 0.91 , with an average of 0.8933 . The $\mathrm{R}^{2}$ value varied from 0.87 to 0.94 , with an average of 0.9033 (Figure 16). The PBIAS values ranged from $-5.71 \%$ for overestimating to $3.66 \%$ for underestimating.

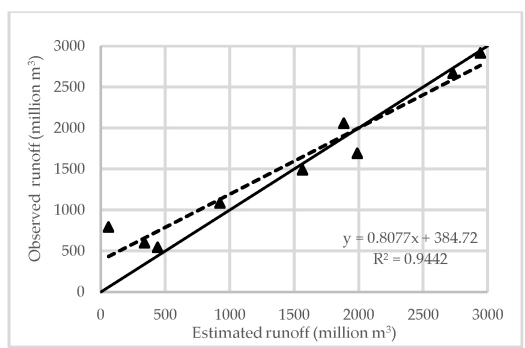

(a)

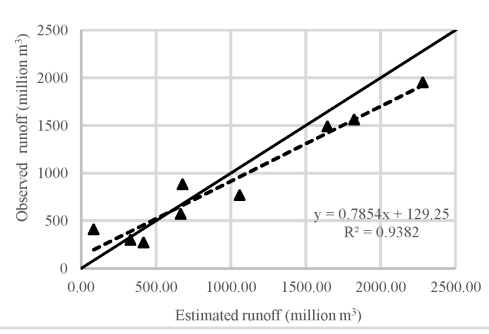

(b)

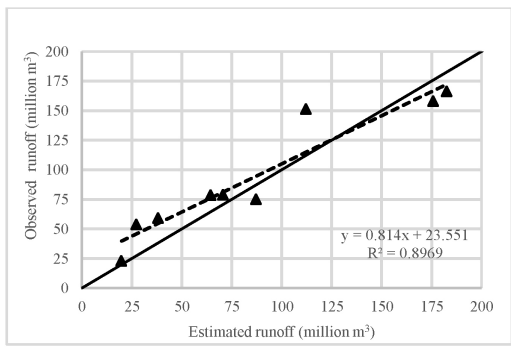

(c)

Figure 16. Relationship between the observed and estimated runoff between 2011 and 2019 at the three stations: (a) E.21 station, (b) E.23 station, and (c) E.6C station.

According to a statistical report of model performance for surface runoff estimation between 2011 and 2019, the derived NSE and $R^{2}$ values were more than 0.87 and the PBIAS value was less than \pm 10 . These results show a very good fit for the surface runoff estimation with a very high correlation between the observed and estimated surface runoff, as suggested by [50] (see Table 3). Thus, it can be concluded that the surface runoff estimation using the SCS-CN method in the current study can be validated with acceptable results.

\subsection{SPI Calculation for Rainfall Condition Identification}

The available historical rainfall data records from 33 years (1987-2019) of the Chaiyaphum meteorological station (Figure 17) were applied to calculate the 12-month SPI index, as shown in Figure 18. The derived SPI values were classified into seven drought types and grouped into three rainfall conditions: dry, normal, and wet years, as summarized in Table 23.

Annual rainfall

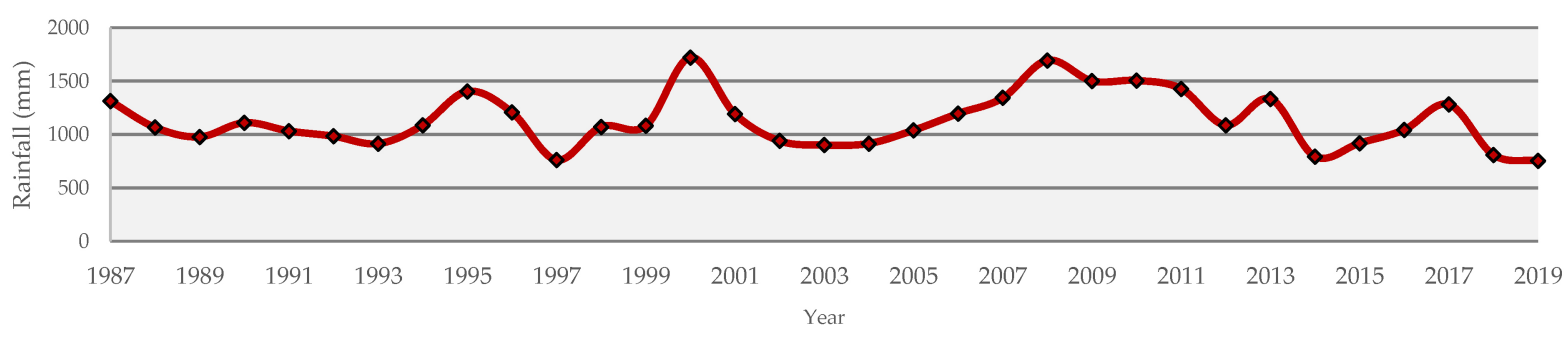

Figure 17. Annual rainfall of Chaiyaphum meteorological station (1987-2019). 
Standardized Precipitation Index (SPI)

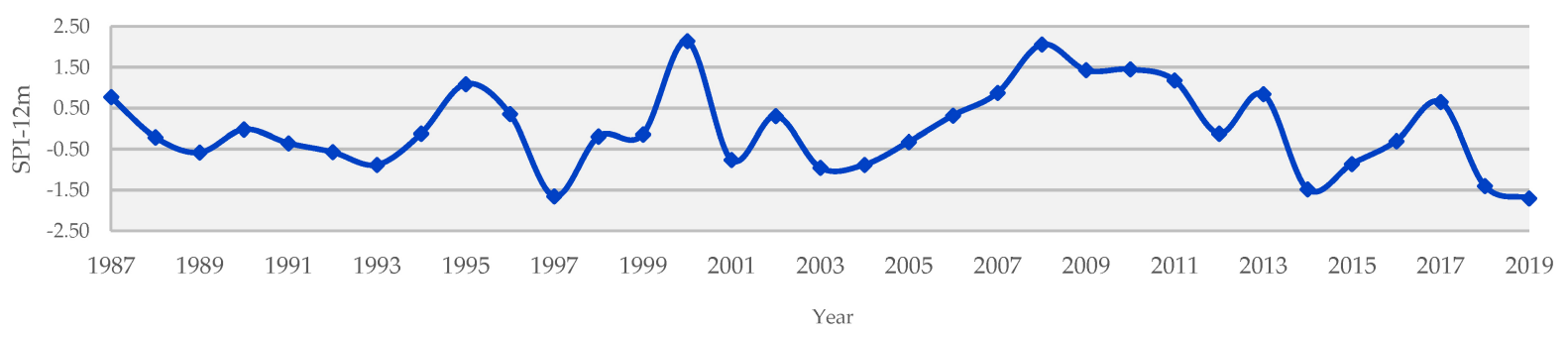

Figure 18. The 12-month SPI values of Chaiyaphum meteorological station (1987 and 2019).

Table 23. The 12-month SPI values, drought classification, and rainfall conditions of Chaiyaphum meteorological station (1987-2019).

\begin{tabular}{|c|c|c|c|c|c|c|c|}
\hline Year & SPI & $\begin{array}{c}\text { Drought } \\
\text { Classification }\end{array}$ & $\begin{array}{l}\text { Rainfall } \\
\text { Conditions }\end{array}$ & Year & SPI & $\begin{array}{c}\text { Drought } \\
\text { Classification } 1\end{array}$ & $\begin{array}{c}\text { Rainfall } \\
\text { Conditions }\end{array}$ \\
\hline 1987 & 0.77 & Mild wet & Wet year & 2004 & -0.88 & Mild drought & Dry year \\
\hline 1988 & -0.21 & Near normal & Normal year & 2005 & -0.32 & Near normal & Normal year \\
\hline 1989 & -0.58 & Mild drought & Dry year & 2006 & 0.32 & Near normal & Normal year \\
\hline 1990 & -0.02 & Near normal & Normal year & 2007 & 0.87 & Mild wet & Wet year \\
\hline 1991 & -0.35 & Near normal & Normal year & 2008 & 2.06 & Extreme wet & Wet year \\
\hline 1992 & -0.57 & Mild drought & Dry year & 2009 & 1.43 & Moderate wet & Wet year \\
\hline 1993 & -0.88 & Mild drought & Dry year & 2010 & 1.45 & Moderate wet & Wet year \\
\hline 1994 & -0.12 & Near normal & Normal year & 2011 & 1.18 & Moderate wet & Wet year \\
\hline 1995 & 1.09 & Moderate wet & Wet year & 2012 & -0.12 & Near normal & Normal year \\
\hline 1996 & 0.36 & Mild wet & Wet year & 2013 & 0.84 & Mild wet & Wet year \\
\hline 1997 & -1.65 & Severe drought & Dry year & 2014 & -1.48 & Moderate drought & Dry year \\
\hline 1998 & -0.19 & Near normal & Normal year & 2015 & -0.86 & Mild drought & Dry year \\
\hline 1999 & -0.14 & Near normal & Normal year & 2016 & -0.30 & Near normal & Normal year \\
\hline 2000 & 2.14 & Extreme wet & Wet year & 2017 & 0.65 & Mild wet & Wet year \\
\hline 2001 & -0.76 & Mild drought & Dry year & 2018 & -1.40 & Moderate drought & Dry year \\
\hline 2002 & 0.31 & Near normal & Normal year & 2019 & -1.70 & Severe drought & Dry year \\
\hline 2003 & -0.95 & Mild drought & Dry year & & & & \\
\hline
\end{tabular}

As a result, the dry years were found to be 2001, 2003, 2004, 2014, 2015, 2018, and 2019. Meanwhile, normal years occurred in 2002, 2005, 2006, 2012, and 2016, and wet years in 2007, 2008, 2009, 2010, 2011, 2013, and 2017.

\subsection{Optimization of LULC Allocation for Flood Mitigation}

Goal programming of multi-objective decision analysis (MODA) was applied to allocate the optimum LULC to minimize surface runoff for flood mitigation based on the average surface runoff coefficient from LULC under three rainfall conditions. In this study, an average of each LULC type was separately extracted from the time series surface runoff between 2001 and 2019 for dry, normal, and wet years, as presented in Table 24. 
Table 24. Runoff coefficient and its average in dry years.

\begin{tabular}{|c|c|c|c|c|}
\hline \multirow{2}{*}{ No. } & \multirow{2}{*}{ LULC Type } & \multicolumn{3}{|c|}{ Average Surface Runoff Coefficient (million $\mathrm{m}^{3} / \mathrm{km}^{2}$ ) } \\
\hline & & Dry Year & Normal Year & Wet Year \\
\hline 1 & $\begin{array}{l}\text { Urban and } \\
\text { built-up areas }\end{array}$ & 0.45 & 1.03 & 1.46 \\
\hline 2 & Paddy fields & 0.44 & 1.01 & 1.44 \\
\hline 3 & Sugarcane & 0.38 & 0.90 & 1.30 \\
\hline 4 & Cassava & 0.45 & 1.00 & 1.41 \\
\hline 5 & Other field crops & 0.39 & 0.88 & 1.31 \\
\hline 6 & Para rubber & 0.37 & 0.83 & 1.30 \\
\hline 7 & $\begin{array}{l}\text { Perennial trees } \\
\text { and orchards }\end{array}$ & 0.38 & 0.84 & 1.31 \\
\hline 8 & Forest land & 0.28 & 0.76 & 1.17 \\
\hline 9 & Waterbodies & 0.49 & 1.04 & 1.51 \\
\hline 10 & Rangeland & 0.42 & 0.92 & 1.36 \\
\hline 11 & $\begin{array}{l}\text { Marshes and } \\
\text { swamps }\end{array}$ & 0.54 & 1.14 & 1.59 \\
\hline 12 & Unused land & 0.46 & 1.02 & 1.41 \\
\hline
\end{tabular}

At the same time, the constraints of goal programming subjected to the change of LULC area in the study area were assigned for optimizing the LULC allocation for flood mitigation based on the historical LULC development between 2010 and 2019. In this study, a 10-year period was chosen to predict LULC data in 2029, 2039, and 2049 based on a period of input data (2010-2019) for transitional area prediction by the Markov Chain model. Accordingly, the changing area of each LULC type was categorized into two groups: Decreased area (paddy fields, cassava, forest land, waterbodies, rangeland, and marshes and swamps) and increased area (urban and built-up areas, sugarcane, other field crops, para rubber, perennial trees and orchards, and unused land) according to the derived transitional change area from the Markov Chain model. Details of the LULC change to minimize surface runoff in 2029, 2039, and 2049 as constraints of goal programming are summarized in Table 25.

Table 25. Existing and predicted areas of LULC in 2029, 2039, and 2049 for constraint setting.

\begin{tabular}{|c|c|c|c|c|c|c|c|}
\hline \multirow[b]{2}{*}{ LULC Types } & \multirow{2}{*}{$\begin{array}{c}\text { Existing } \\
\text { Area } \\
\left(\mathbf{k m}^{2}\right)\end{array}$} & \multicolumn{2}{|c|}{ LULC in $2029\left(\mathrm{~km}^{2}\right)$} & \multicolumn{2}{|c|}{ LULC in $2039\left(\mathrm{~km}^{2}\right)$} & \multicolumn{2}{|c|}{ LULC in $2049\left(\mathrm{~km}^{2}\right)$} \\
\hline & & $\begin{array}{l}\text { Predicted } \\
\text { Area }\end{array}$ & Remark & $\begin{array}{l}\text { Predicted } \\
\text { Area }\end{array}$ & Remark & $\begin{array}{l}\text { Predicted } \\
\text { Area }\end{array}$ & Remark \\
\hline Urban and built-up areas $\left(\mathrm{X}_{1}\right)$ & 65.84 & 77.38 & $0.17 \%$ increase & 88.31 & $0.34 \%$ increase & 99.03 & $0.50 \%$ increase \\
\hline Paddy fields $\left(\mathrm{X}_{2}\right)$ & 2012.16 & 1947.12 & $0.03 \%$ decrease & 1879.30 & $0.06 \%$ decrease & 1812.16 & $0.09 \%$ decrease \\
\hline Sugarcane $\left(X_{3}\right)$ & 306.85 & 424.95 & $0.38 \%$ increase & 520.31 & $0.69 \%$ increase & 599.36 & $0.95 \%$ increase \\
\hline Cassava $\left(\mathrm{X}_{4}\right)$ & 489.91 & 408.26 & $0.16 \%$ decrease & 358.65 & $0.26 \%$ decrease & 330.32 & $0.32 \%$ decrease \\
\hline Other field crops $\left(X_{5}\right)$ & 6.19 & 7.72 & $0.27 \%$ increase & 9.4525 & $0.52 \%$ increase & 11.26 & $0.81 \%$ increase \\
\hline Para rubber $\left(X_{6}\right)$ & 97.03 & 134.43 & $0.38 \%$ increase & 152.02 & $0.56 \%$ increase & 164.41 & $0.69 \%$ increase \\
\hline Perennial trees and orchards $\left(X_{7}\right)$ & 88.95 & 125.36 & $0.40 \%$ increase & 160.12 & $0.80 \%$ increase & 193.13 & $1.17 \%$ increase \\
\hline Forest land $\left(\mathrm{X}_{8}\right)$ & 481.30 & 382.63 & $0.20 \%$ decrease & 304.44 & $0.36 \%$ decrease & 242.23 & $0.49 \%$ decrease \\
\hline Waterbodies $\left(X_{9}\right)$ & 53.30 & 49.52 & $0.07 \%$ decrease & 47.01 & $0.13 \%$ decrease & 42.78 & $0.19 \%$ decrease \\
\hline Rangeland $\left(X_{10}\right)$ & 71.65 & 70.54 & $0.01 \%$ decrease & 69.91 & $0.02 \%$ decrease & 66.05 & $0.07 \%$ decrease \\
\hline Marshes and swamps $\left(X_{11}\right)$ & 27.73 & 25.13 & $0.09 \%$ decrease & 24.66 & $0.11 \%$ decrease & 21.61 & $0.07 \%$ decrease \\
\hline Unused land $\left(\mathrm{X}_{12}\right)$ & 93.31 & 141.20 & $0.51 \%$ increase & 180.07 & $0.93 \%$ increase & 212.00 & $1.27 \%$ increase \\
\hline Total & 3794.22 & 3794.22 & & 3794.22 & & 3794.22 & \\
\hline
\end{tabular}

Based on the linearity of the objective function and constraints, the objective functions of the surface runoff minimization problem for optimizing LULC allocation in dry, normal, and wet years were formulated as shown in Equations (17)-(19).

$$
\begin{gathered}
\operatorname{Min}(Z)=0.45 X_{1}+0.44 X_{2}+0.38 X_{3}+0.45 X_{4}+0.39 X_{5}+0.37 X_{6}+0.38 X_{7}+0.28 X_{8}+0.49 X_{9}+ \\
0.42 X_{10}+0.54 X_{11}+0.46 X_{12} \\
\operatorname{Min}(Z)=1.03 X_{1}+1.01 X_{2}+0.90 X_{3}+1.00 X_{4}+0.88 X_{5}+0.83 X_{6}+0.84 X_{7}+0.76 X_{8}+1.04 X_{9}+ \\
0.92 X_{10}+1.14 X_{11}+1.02 X_{12}
\end{gathered}
$$




$$
\begin{gathered}
\operatorname{Min}(\mathrm{Z})=1.46 \mathrm{X}_{1}+1.44 \mathrm{X}_{2}+1.30 \mathrm{X}_{3}+1.41 \mathrm{X}_{4}+1.31 \mathrm{X}_{5}+1.30 \mathrm{X}_{6}+1.31 \mathrm{X}_{7}+1.17 \mathrm{X}_{8}+1.51 \mathrm{X}_{9}+ \\
1.36 \mathrm{X}_{10}+1.59 \mathrm{X}_{11}+1.41 \mathrm{X}_{12}
\end{gathered}
$$

The constraints for optimizing LULC allocation in 2029, 2039, and 2049 to minimize runoff under three rainfall conditions using objective functions (Equations (17)-(19)) are summarized in Table 26. Additionally, two mandatory constraints, as described by Equations (11) and (14) in Section 3.5.2, were set under the three rainfall conditions as follows:

Table 26. Summary of the constraint setting for each LULC type in 2029, 2039, and 2049.

\begin{tabular}{lccc}
\hline \multirow{2}{*}{ LULC Types } & \multicolumn{3}{c}{ Constraints Setting for Each LULC Type } \\
\cline { 2 - 4 } & Constrain in 2029 & Constrain in 2039 & Constrain in 2049 \\
\hline Urban and built-up areas $\left(X_{1}\right)$ & $65.84 \leq X_{1} \leq 77.38$ & $65.84 \leq X_{1} \leq 88.31$ & $65.84 \leq X_{1} \leq 99.03$ \\
Paddy fields $\left(X_{2}\right)$ & $2012.16 \geq X_{2} \geq 1947.12$ & $2012.16 \geq X_{2} \geq 1879.30$ & $2012.16 \geq X_{2} \geq 1812.16$ \\
Sugarcane $\left(X_{3}\right)$ & $306.85 \leq X_{3} \leq 424.95$ & $306.85 \leq X_{3} \leq 520.31$ & $306.85 \leq X_{3} \leq 599.36$ \\
Cassava $\left(X_{4}\right)$ & $489.91 \geq X_{4} \geq 408.26$ & $489.91 \geq X_{4} \geq 358.65$ & $489.91 \geq X_{4} \geq 330.32$ \\
Other field crops $\left(X_{5}\right)$ & $6.19 \leq X_{5} \leq 7.72$ & $6.19 \leq X_{5} \leq 9.45$ & $6.19 \leq X_{5} \leq 11.26$ \\
Para rubber $\left(X_{6}\right)$ & $97.03 \leq X_{6} \leq 134.43$ & $97.03 \leq X_{6} \leq 152.02$ & $97.03 \leq X_{6} \leq 164.41$ \\
Perennial trees and orchards $\left(X_{7}\right)$ & $88.95 \leq X_{7} \leq 125.36$ & $88.95 \leq X_{7} \leq 160.12$ & $88.95 \leq X_{7} \leq 193.13$ \\
Forest land $\left(X_{8}\right)$ & $481.30 \geq X_{8} \geq 382.63$ & $481.30 \geq X_{8} \geq 304.44$ & $481.30 \geq X_{8} \geq 242.23$ \\
Waterbodies $\left(X_{9}\right)$ & $53.30 \geq X_{9} \geq 49.52$ & $53.30 \geq X_{9} \geq 47.01$ & $53.30 \geq X_{9} \geq 42.78$ \\
Rangeland $\left(X_{10}\right)$ & $71.65 \geq X_{10} \geq 70.54$ & $71.65 \geq X_{10} \geq 69.91$ & $71.65 \geq X_{10} \geq 66.05$ \\
Marshes and swamps $\left(X_{11}\right)$ & $27.73 \geq X_{11} \geq 25.13$ & $27.73 \geq X_{11} \geq 24.66$ & $27.73 \geq X_{11} \geq 21.61$ \\
Unused land $\left(X_{12}\right)$ & $93.31 \leq X_{12} \leq 141.20$ & $93.31 \leq X_{12} \leq 180.07$ & $93.31 \leq X_{12} \leq 211.89$ \\
\hline
\end{tabular}

The first mandatory constraint, related to the area of all land use classes, must be equal to the allowable area of $3794.22 \mathrm{~km}^{2}$, as shown in Equation (20).

$$
\mathrm{X}_{1}+\mathrm{X}_{2}+\mathrm{X}_{3}+\mathrm{X}_{4}+\mathrm{X}_{5}+\mathrm{X}_{6}+\mathrm{X}_{7}+\mathrm{X}_{8}+\mathrm{X}_{9}+\mathrm{X}_{10}+\mathrm{X}_{11}+\mathrm{X}_{12}=3794.22
$$

The second mandatory constraint is related to the non-negative variable. The area of each land use class should be more than or equal to $0 \mathrm{~km}^{2}$, as shown in Equation (21).

$$
\mathrm{X}_{1}+\mathrm{X}_{2}+\mathrm{X}_{3}+\mathrm{X}_{4}+\mathrm{X}_{5}+\mathrm{X}_{6}+\mathrm{X}_{7}+\mathrm{X}_{8}+\mathrm{X}_{9}+\mathrm{X}_{10}+\mathrm{X}_{11}+\mathrm{X}_{12} \geq 0
$$

The objective functions for dry, normal, and wet years (Equations (17)-(19)) were then transformed into a goal programming form as follows:

$$
\mathrm{f}_{\mathrm{k}}(\mathrm{x})+\mathrm{d}_{\mathrm{k}}^{-}-\mathrm{d}_{\mathrm{k}}^{+}=\mathrm{a}_{\mathrm{k}}
$$

The results of the optimized LULC allocation to minimize surface runoff for flood mitigation in 2029, 2039, and 2049 under the three rainfall conditions (dry, normal, and wet years) are presented in Tables 27-29.

In dry years, the significant increasing LULC type after optimizing LULC allocation in 2029, 2039, and 2049 were sugarcane, with approximately 80, 148, and $210 \mathrm{~km}^{2}$. In contrast, the significantly decreasing LULC type was cassava, with approximately 82,133 , and $200 \mathrm{~km}^{2}$. Additionally, the annual surface runoff decreased in 2029, 2039, and 2049 by $12.95,20.78$, and 27.89 million $\mathrm{m}^{3}$, or approximately $0.82 \%, 1.32 \%$, and $1.77 \%$ of total estimated surface runoff in 2019 (See Table 27).

Meanwhile, the most increased LULC area under the normal year was also sugarcane, with an area of approximately 79, 145, and $205 \mathrm{~km}^{2}$. In contrast, the significant decreasing LULC types were paddy fields, with approximately 65,133 , and $200 \mathrm{~km}^{2}$, and sugarcane, with an area of approximately 82,130 , and $160 \mathrm{~km}^{2}$. Additionally, the annual surface runoff decreased in 2029,2039 , and 2049 by $21.34,37.59$, and 52.31 million $\mathrm{m}^{3}$, or $0.59 \%, 1.03 \%$, and $1.43 \%$ of the total estimated surface runoff in 2019 (see Table 28). 
Table 27. Optimization of LULC allocation to minimize surface runoff in dry years.

\begin{tabular}{|c|c|c|c|c|c|c|c|c|}
\hline \multirow[b]{2}{*}{ LULC Types } & \multicolumn{2}{|c|}{2019} & \multicolumn{2}{|c|}{2029} & \multicolumn{2}{|c|}{2039} & \multicolumn{2}{|c|}{2049} \\
\hline & $\begin{array}{c}\text { Area of } \\
\text { LULC }\end{array}$ & $\begin{array}{l}\text { Surface } \\
\text { Runoff }\end{array}$ & $\begin{array}{l}\text { Allocated } \\
\text { LULC }\end{array}$ & $\begin{array}{l}\text { Surface } \\
\text { Runoff }\end{array}$ & $\begin{array}{l}\text { Allocated } \\
\text { LULC }\end{array}$ & $\begin{array}{l}\text { Surface } \\
\text { Runoff }\end{array}$ & $\begin{array}{l}\text { Allocated } \\
\text { LULC }\end{array}$ & $\begin{array}{l}\text { Surface } \\
\text { Runoff }\end{array}$ \\
\hline Urban and built-up areas & 65.84 & 29.84 & 65.84 & 29.84 & 65.84 & 29.84 & 65.84 & 29.84 \\
\hline Paddy fields & 2012.16 & 942.32 & 1947.12 & 856.54 & 1879.30 & 826.71 & 1812.16 & 797.17 \\
\hline Sugarcane & 306.85 & 70.96 & 387.24 & 148.87 & 454.94 & 174.90 & 517.13 & 198.81 \\
\hline Cassava & 489.91 & 242.89 & 408.26 & 184.27 & 359.65 & 162.33 & 330.32 & 149.09 \\
\hline Other field crops & 6.19 & 1.75 & 6.19 & 2.39 & 6.19 & 2.39 & 6.19 & 2.39 \\
\hline Para rubber & 97.03 & 19.65 & 134.43 & 49.86 & 152.02 & 56.39 & 164.41 & 60.99 \\
\hline Perennial trees and orchards & 88.95 & 25.76 & 125.36 & 47.40 & 160.12 & 60.54 & 193.13 & 73.02 \\
\hline Forest land & 481.30 & 159.21 & 481.30 & 136.03 & 481.30 & 136.03 & 481.30 & 136.03 \\
\hline Waterbodies & 53.30 & 23.81 & 49.52 & 24.29 & 47.01 & 23.06 & 42.78 & 20.99 \\
\hline Rangeland & 71.65 & 23.23 & 70.54 & 29.36 & 69.91 & 29.10 & 66.05 & 27.49 \\
\hline Marshes and swamps & 27.73 & 12.69 & 25.13 & 13.59 & 24.66 & 13.34 & 21.61 & 11.69 \\
\hline Unused land & 93.31 & 25.85 & 93.31 & 42.57 & 93.31 & 42.57 & 93.31 & 42.57 \\
\hline Total & 3794.22 & 1577.96 & 3794.22 & 1565.01 & 3794.22 & 1557.18 & 3794.22 & $\mathbf{1 5 5 0 . 0 7}$ \\
\hline
\end{tabular}

Note: Area of LULC in kilometers squared, surface runoff in million meters cubed, and allocated LULC in kilometers squared.

Table 28. Optimization of LULC allocation to minimize surface runoff in normal years.

\begin{tabular}{|c|c|c|c|c|c|c|c|c|}
\hline \multirow[b]{2}{*}{ LULC Types } & \multicolumn{2}{|c|}{2019} & \multicolumn{2}{|c|}{2029} & \multicolumn{2}{|c|}{2039} & \multicolumn{2}{|c|}{2049} \\
\hline & $\begin{array}{l}\text { Area of } \\
\text { LULC }\end{array}$ & $\begin{array}{l}\text { Surface } \\
\text { Runoff }\end{array}$ & $\begin{array}{l}\text { Allocated } \\
\text { LULC }\end{array}$ & $\begin{array}{l}\text { Surface } \\
\text { Runoff }\end{array}$ & $\begin{array}{l}\text { Allocated } \\
\text { LULC }\end{array}$ & $\begin{array}{l}\text { Surface } \\
\text { Runoff }\end{array}$ & $\begin{array}{l}\text { Allocated } \\
\text { LULC }\end{array}$ & $\begin{array}{l}\text { Surface } \\
\text { Runoff }\end{array}$ \\
\hline Urban and built-up areas & 65.84 & 67.58 & 65.84 & 67.58 & 65.84 & 67.58 & 65.84 & 67.58 \\
\hline Paddy fields & 2012.16 & 2038.95 & 1947.12 & 1973.05 & 1879.30 & 1904.33 & 1812.16 & 1836.29 \\
\hline Sugarcane & 306.85 & 277.30 & 385.71 & 348.56 & 451.67 & 408.18 & 512.06 & 462.76 \\
\hline Cassava & 489.91 & 489.07 & 408.26 & 407.56 & 359.65 & 359.03 & 330.32 & 329.75 \\
\hline Other field crops & 6.19 & 5.43 & 7.72 & 6.78 & 9.45 & 8.30 & 11.26 & 9.88 \\
\hline Para rubber & 97.03 & 80.36 & 134.43 & 111.33 & 152.02 & 125.89 & 164.41 & 136.16 \\
\hline Perennial trees and orchards & 88.95 & 74.28 & 125.36 & 104.68 & 160.12 & 133.70 & 193.13 & 161.27 \\
\hline Forest land & 481.30 & 366.03 & 481.30 & 366.03 & 481.30 & 366.03 & 481.30 & 366.03 \\
\hline Waterbodies & 53.30 & 55.23 & 49.52 & 51.30 & 47.01 & 48.70 & 42.78 & 44.33 \\
\hline Rangeland & 71.65 & 65.95 & 70.54 & 64.93 & 69.91 & 64.35 & 66.05 & 60.80 \\
\hline Marshes and swamps & 27.73 & 31.56 & 25.13 & 28.60 & 24.66 & 28.06 & 21.61 & 24.59 \\
\hline Unused land & 93.31 & 95.33 & 93.31 & 95.33 & 93.31 & 95.33 & 93.31 & 95.33 \\
\hline Total & 3794.22 & 3647.07 & 3794.22 & 3625.72 & 3794.22 & 3609.48 & 3794.22 & 3594.76 \\
\hline
\end{tabular}

Note: Area of LULC in kilometers squared, surface runoff in million meters cubed, and allocated LULC in kilometers squared.

Table 29. Optimization of LULC allocation to minimize surface runoff in wet years.

\begin{tabular}{|c|c|c|c|c|c|c|c|c|}
\hline \multirow[b]{2}{*}{ LULC Types } & \multicolumn{2}{|c|}{2019} & \multicolumn{2}{|c|}{2029} & \multicolumn{2}{|c|}{2039} & \multicolumn{2}{|c|}{2049} \\
\hline & $\begin{array}{c}\text { Area of } \\
\text { LULC }\end{array}$ & $\begin{array}{l}\text { Surface } \\
\text { Runoff }\end{array}$ & $\begin{array}{c}\text { Allocated } \\
\text { LULC }\end{array}$ & $\begin{array}{l}\text { Surface } \\
\text { Runoff }\end{array}$ & $\begin{array}{l}\text { Allocated } \\
\text { LULC }\end{array}$ & $\begin{array}{l}\text { Surface } \\
\text { Runoff }\end{array}$ & $\begin{array}{l}\text { Allocated } \\
\text { LULC }\end{array}$ & $\begin{array}{l}\text { Surface } \\
\text { Runoff }\end{array}$ \\
\hline Urban and built-up areas & 65.84 & 95.82 & 65.84 & 95.82 & 65.84 & 95.82 & 65.84 & 95.82 \\
\hline Paddy fields & 2012.16 & 2889.20 & 1947.12 & 2795.81 & 1879.30 & 2698.43 & 1812.16 & 2602.02 \\
\hline Sugarcane & 306.85 & 400.38 & 422.14 & 552.77 & 520.31 & 678.91 & 599.36 & 782.05 \\
\hline Cassava & 489.91 & 688.86 & 408.26 & 574.05 & 358.65 & 505.70 & 330.32 & 464.46 \\
\hline Other field crops & 6.19 & 8.09 & 6.19 & 8.09 & 9.45 & 12.36 & 11.26 & 14.71 \\
\hline Para rubber & 97.03 & 126.18 & 134.43 & 174.82 & 152.02 & 197.69 & 164.41 & 213.81 \\
\hline Perennial trees and orchards & 88.95 & 116.42 & 88.95 & 116.42 & 90.48 & 119.73 & 103.83 & 138.51 \\
\hline Forest land & 481.30 & 563.35 & 481.30 & 563.35 & 481.30 & 563.35 & 481.30 & 563.35 \\
\hline Waterbodies & 53.30 & 80.24 & 50.02 & 74.55 & 48.01 & 70.77 & 43.78 & 64.41 \\
\hline Rangeland & 71.65 & 97.12 & 70.54 & 95.60 & 69.91 & 94.76 & 66.05 & 89.52 \\
\hline Marshes and swamps & 27.73 & 44.10 & 26.13 & 39.97 & 25.66 & 39.21 & 22.61 & 34.37 \\
\hline Unused land & 93.31 & 131.74 & 93.31 & 131.74 & 93.31 & 131.74 & 93.31 & 131.74 \\
\hline Total & 3794.22 & 5241.52 & 3794.22 & 5223.01 & 3794.22 & 5208.46 & 3794.22 & 5194.79 \\
\hline
\end{tabular}


In the meantime, the most increasing LULC type in wet years was sugarcane, with an area of approximately 115, 213, and $293 \mathrm{~km}^{2}$, respectively. Conversely, the significant decreasing LULC types were paddy fields, with approximately 65,133 , and $200 \mathrm{~km}^{2}$, and sugarcane, with approximately 82,131 , and $160 \mathrm{~km}^{2}$. Additionally, the annual surface runoff decreased in 2029, 2039, and 2049 by 18.52, 33.06, and 46.73 million $\mathrm{m}^{3}$, or approximately $0.35 \%, 0.63 \%$, and $0.89 \%$ from the total estimated surface runoff in 2019 (see Table 29).

According to these results, the optimized LULC allocation data in 2049 of the dry, normal and wet years were suitable for flood mitigation according to surface runoff reduction. The surface runoff under dry, normal and wet years in 2049 reduced by approximately $27.89,52.31$, and 46.73 million $\mathrm{m}^{3}$ (Figure 19).

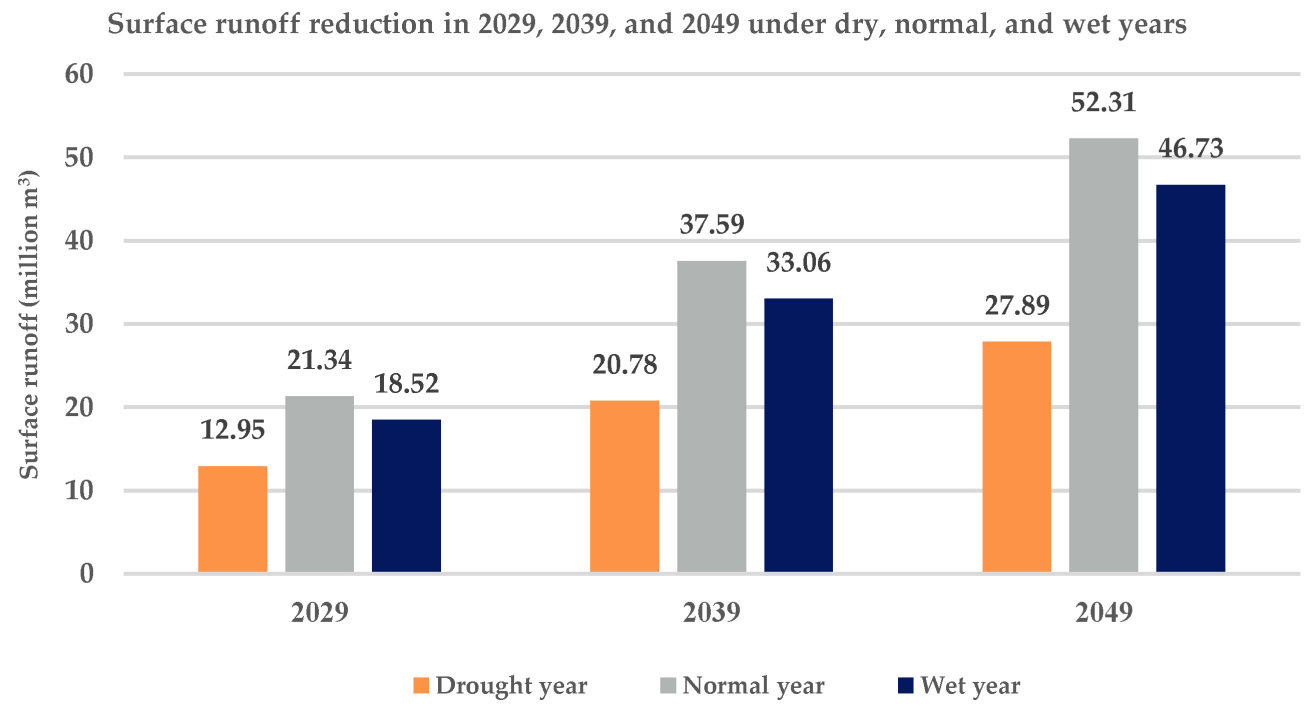

Figure 19. Surface runoff reduction in 2029, 2039, and 2049 in dry, normal, and wet years.

Moreover, notable reductions in annual surface runoff under the three rainfall conditions occurred in paddy fields and cassava to sugarcane, para rubber, and perennial trees and orchards after optimization, which changed the hydrological properties. Paddy and cassava fields provided higher runoff coefficients than sugarcane, rubber, and perennial trees and orchards (see Tables 27-29). This finding is consistent with previous studies [19,20,71].

The deviation in annual surface runoff after minimization by goal programming is presented in Table 30. As a result, the percentage deviation from the goal varied from $-1.77 \%$ to $-0.35 \%$, which is acceptable.

Table 30. Deviation of annual surface runoff after minimization by goal programming.

\begin{tabular}{|c|c|c|c|c|c|c|c|c|c|}
\hline \multirow{3}{*}{ Items } & \multicolumn{9}{|c|}{ Surface Runoff Minimization (million $\mathrm{m}^{3}$ ) } \\
\hline & \multicolumn{3}{|c|}{ Dry Years } & \multicolumn{3}{|c|}{ Normal Years } & \multicolumn{3}{|c|}{ Wet Years } \\
\hline & 2029 & 2039 & 2049 & 2029 & 2039 & 2049 & 2029 & 2039 & 2049 \\
\hline $\begin{array}{l}\text { Goal of annual surface runoff } \\
\left(\text { million } \mathrm{m}^{3}\right)\end{array}$ & 1577.96 & 1577.96 & 1577.96 & 3647.07 & 3647.07 & 3647.07 & 5241.52 & 5241.52 & 5241.52 \\
\hline $\begin{array}{l}\text { Annual surface runoff after } \\
\text { optimization (million } \mathrm{m}^{3} \text { ) }\end{array}$ & 1565.01 & 1557.18 & 1550.07 & 3625.72 & 3609.48 & 3594.76 & 5223.01 & 5208.46 & 5194.79 \\
\hline Deviation from goal (million $\mathrm{m}^{3}$ ) & -12.95 & -20.78 & -27.89 & -21.34 & -37.59 & -52.31 & -18.52 & -33.06 & -46.73 \\
\hline Deviation from goal $(\%)$ & -0.82 & -1.32 & -1.77 & -0.59 & -1.03 & -1.43 & -0.35 & -0.63 & -0.89 \\
\hline
\end{tabular}

\subsection{Mapping of LULC Allocation for Flood Mitigation}

LULC data in 2019 and the derived optimum local parameter of the CLUE-S model were applied to map the suitable LULC allocation to minimize surface runoff for flood mitigation in 2049 of the three rainfall conditions. The conversion matrix for each possible 
LULC type change in 2049 was based on transitional LULC change between 2010 and 2019, as shown in Table 31. Meanwhile, the elasticity values were assigned based on the transition probability matrix of LULC change between 2019 and 2049 from the Markov Chain model, as shown in Table 32. Thus, the elasticity values of the urban and built-up areas, paddy fields, sugarcane, cassava, other field crops, para rubber, perennial trees and orchards, forest land, waterbodies, rangeland, marshes and swamps, and unused land for LULC prediction in 2049 were $1.00,0.83,0.83,0.31,0.97,0.52,0.96,0.70,0.76,0.71,0.76$, and 0.87 , respectively.

Table 31. Conversion matrix of the possible change in 2029, 2039, and 2049.

\begin{tabular}{|c|c|c|c|c|c|c|c|c|c|c|c|c|c|}
\hline & \multirow{2}{*}{ LULC Types } & \multicolumn{12}{|c|}{ LULC Type Possible Change in 2029, 2039, and 2049} \\
\hline & & UR & PA & SU & CA & FC & PR & PO & FO & WA & RA & MA & UL \\
\hline \multirow{12}{*}{ 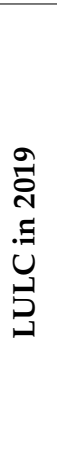 } & Urban and built-up areas (UR) & 1 & 0 & 0 & 0 & 0 & 0 & 0 & 0 & 0 & 0 & 0 & 0 \\
\hline & Paddy fields (PA) & 0 & 1 & 1 & 1 & 0 & 0 & 0 & 0 & 0 & 1 & 1 & 0 \\
\hline & Sugarcane (SU) & 1 & 0 & 1 & 1 & 0 & 1 & 1 & 0 & 0 & 0 & 0 & 0 \\
\hline & Cassava (CA) & 1 & 1 & 1 & 1 & 0 & 1 & 1 & 0 & 0 & 1 & 0 & 1 \\
\hline & Other field crops (FC) & 0 & 0 & 0 & 0 & 1 & 0 & 0 & 0 & 0 & 0 & 0 & 0 \\
\hline & Para rubber $(\mathrm{PR})$ & 0 & 0 & 1 & 1 & 1 & 1 & 0 & 0 & 0 & 0 & 0 & 1 \\
\hline & Perennial trees and orchards (PO) & 0 & 0 & 1 & 1 & 0 & 0 & 1 & 0 & 0 & 0 & 0 & 0 \\
\hline & Forest land (FO) & 0 & 0 & 0 & 0 & 0 & 1 & 1 & 1 & 0 & 1 & 0 & 1 \\
\hline & Waterbodies (WA) & 0 & 0 & 0 & 0 & 0 & 0 & 0 & 0 & 1 & 0 & 1 & 0 \\
\hline & Rangeland (RA) & 0 & 1 & 0 & 1 & 0 & 0 & 1 & 0 & 0 & 1 & 0 & 0 \\
\hline & Marshes and swamps (MA) & 0 & 1 & 1 & 0 & 0 & 0 & 1 & 0 & 0 & 0 & 1 & 0 \\
\hline & Unused land (UL) & 1 & 0 & 0 & 0 & 0 & 0 & 0 & 0 & 0 & 0 & 0 & 1 \\
\hline
\end{tabular}

Table 32. Elasticity of LULC change for LULC prediction between 2019 and 2049.

\begin{tabular}{|c|c|c|c|c|c|c|c|c|c|c|c|c|c|}
\hline & \multirow{2}{*}{ LULC Types } & \multicolumn{12}{|c|}{ LULC Type Possible Change in 2049} \\
\hline & & UR & PA & SU & $\mathrm{CA}$ & FC & PR & PO & FO & WA & RA & MA & UL \\
\hline \multirow{12}{*}{ 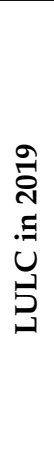 } & Urban and built-up areas (UR) & 1.00 & - & - & - & - & - & - & - & - & - & - & - \\
\hline & Paddy fields (PA) & - & 0.83 & 0.08 & 0.04 & - & - & 0.03 & - & - & - & 0.01 & - \\
\hline & Sugarcane (SU) & 0.02 & 0.03 & 0.83 & 0.09 & - & 0.01 & 0.02 & - & - & - & - & 0.01 \\
\hline & Cassava (CA) & 0.03 & 0.12 & 0.31 & 0.31 & - & 0.11 & 0.03 & - & - & 0.01 & - & 0.08 \\
\hline & Other field crops (FC) & - & - & - & - & 0.97 & - & 0.02 & - & - & - & - & - \\
\hline & Para rubber (PR) & 0.01 & 0.02 & 0.05 & 0.18 & 0.02 & 0.52 & 0.07 & - & - & - & - & 0.13 \\
\hline & Perennial trees and orchards (PO) & - & 0.01 & 0.02 & 0.01 & - & - & 0.96 & - & - & - & - & - \\
\hline & Forest land (FO) & 0.01 & 0.01 & 0.03 & 0.04 & - & 0.10 & 0.04 & 0.70 & - & 0.01 & - & 0.04 \\
\hline & Waterbodies (WA) & 0.01 & 0.10 & 0.07 & 0.03 & - & - & 0.01 & - & 0.76 & - & 0.01 & - \\
\hline & Rangeland (RA) & - & 0.21 & 0.02 & 0.03 & - & - & 0.02 & - & - & 0.71 & - & - \\
\hline & Marshes and swamps (MA) & 0.01 & 0.06 & 0.08 & 0.03 & - & - & 0.05 & - & - & - & 0.77 & - \\
\hline & Unused land (UL) & 0.02 & 0.08 & 0.01 & 0.01 & - & - & 0.01 & - & - & - & - & 0.87 \\
\hline
\end{tabular}

In addition, the optimized LULC allocation in 2049 in dry, normal, and wet years (see Tables 27-29) was applied to calculate the annual land requirement between 2019 and 2049 for LULC prediction.

The spatial distribution of the LULC allocation maps in 2049 for dry, normal, and wet years is displayed in Figure 20. Meanwhile, the area and percentage of LULC type in 2049 for dry, normal, and wet years are summarized in Table 33. The derived map of suitable LULC allocation to minimize surface runoff for flood mitigation of dry, normal, and wet years was further applied to evaluate economic and ecosystem service values and change. 


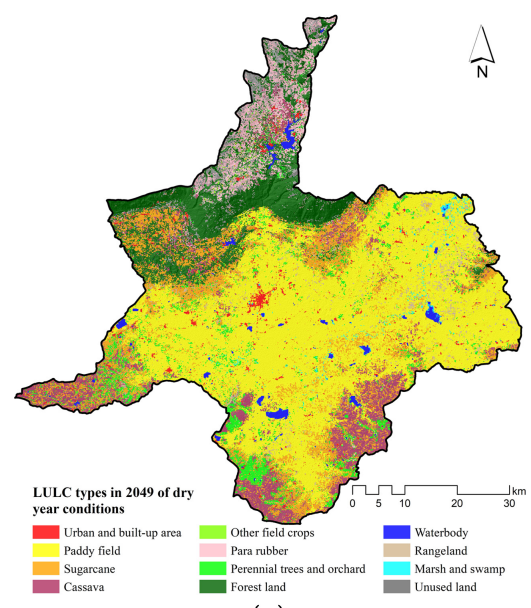

(a)

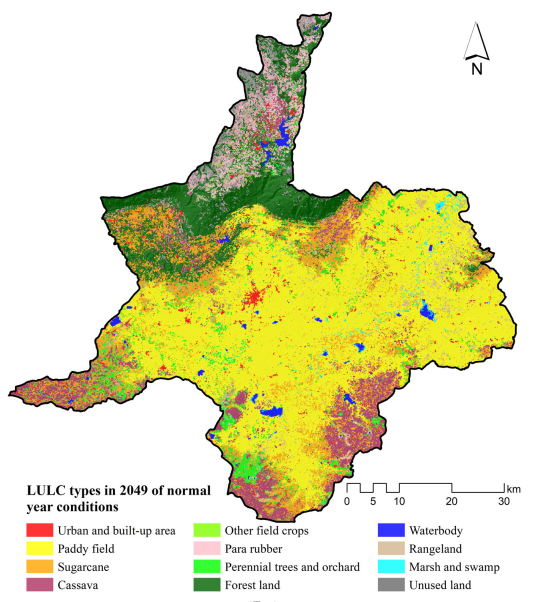

(b)

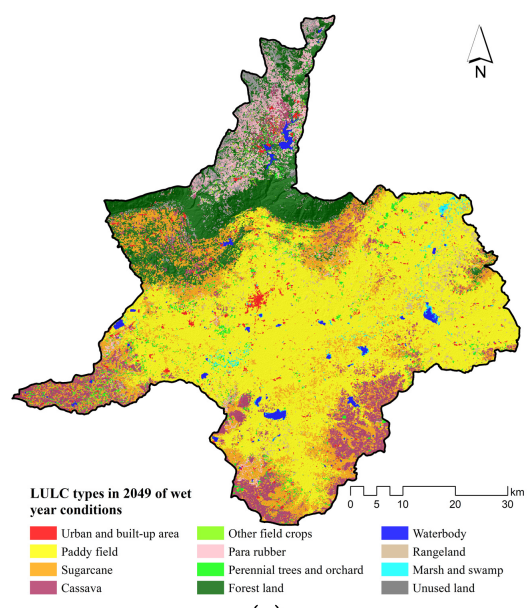

(c)

Figure 20. Spatial distribution of predicted LULC data in 2049: (a) Dry years, (b) normal years, and (c) wet years.

Table 33. Area and percentage of optimized LULC allocation to mitigate flood in 2049 in dry, normal, and wet years.

\begin{tabular}{lcccccc}
\hline \multirow{2}{*}{ LULC Types } & \multicolumn{2}{c}{ Dry Year } & \multicolumn{2}{c}{ Normal Year } & \multicolumn{2}{c}{ Wet Year } \\
\cline { 2 - 6 } & Area $\left.\mathbf{( k m}^{\mathbf{2}}\right)$ & Percentage & Area $\left.\mathbf{( k m}^{\mathbf{2}}\right)$ & Percentage & Area $\left.\mathbf{( k m}^{\mathbf{2}}\right)$ & Percentage \\
\hline Urban and built-up areas & 65.84 & 1.74 & 65.84 & 1.74 & 65.84 & 1.74 \\
Paddy fields & 1812.14 & 47.76 & 1812.14 & 47.76 & 1812.18 & 47.76 \\
Sugarcane & 517.12 & 13.63 & 517.12 & 13.63 & 599.34 & 15.80 \\
Cassava & 330.33 & 8.71 & 330.33 & 8.71 & 330.34 & 8.71 \\
Other field crops & 6.19 & 0.16 & 6.19 & 0.16 & 11.25 & 0.30 \\
Para rubber & 164.42 & 4.33 & 164.42 & 4.33 & 164.41 & 4.33 \\
Perennial trees and orchards & 193.17 & 5.09 & 193.17 & 5.09 & 103.88 & 2.74 \\
Forest land & 481.26 & 12.68 & 481.26 & 12.68 & 481.3 & 12.69 \\
Waterbodies & 42.77 & 1.13 & 42.77 & 1.13 & 43.78 & 1.15 \\
Rangeland & 66.07 & 1.74 & 66.07 & 1.74 & 66.01 & 1.74 \\
Marshes and swamps & 21.57 & 0.57 & 21.57 & 0.57 & 22.56 & 0.59 \\
Unused land & 93.3 & 2.46 & 93.3 & 2.46 & 93.28 \\
\hline Total & $\mathbf{3 7 9 4 . 2 2}$ & $\mathbf{1 0 0 . 0 0}$ & $\mathbf{3 7 9 4 . 2 2}$ & $\mathbf{1 0 0 . 0 0}$ & $\mathbf{3 7 9 4 . 2 2}$ & $\mathbf{1 0 0 . 0 0}$ \\
\hline
\end{tabular}

\subsection{Economic Value Evaluation and Change}

The actual LULC data in 2019 and suitable LULC data in 2049 for flood mitigation in dry, normal, and wet years (Table 34) were applied to evaluate future economic values using the PV model (Table 35), and the results are shown in Table 36 and Figure 21.

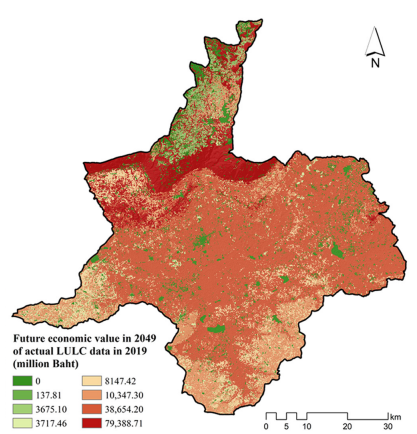

(a)

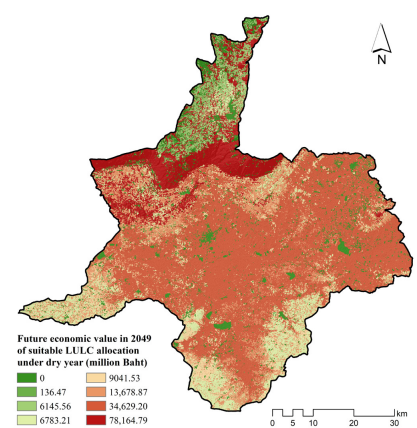

(b)

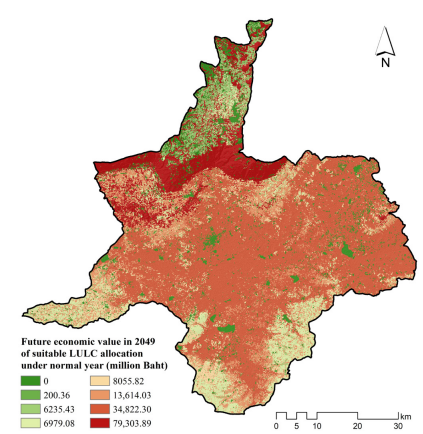

(c)

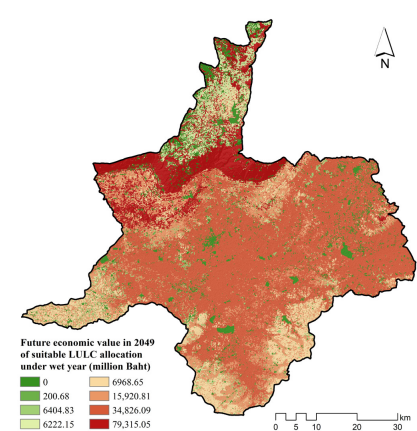

(d)

Figure 21. Spatial distribution of economic value in 2049: (a) Actual LULC 2019, (b) dry years, (c) normal years, and $(\mathbf{d})$ wet years. 
Table 34. Areas of actual LULC in 2019 and suitable LULC allocation for flood mitigation in 2049 in dry, normal, and wet years.

\begin{tabular}{|c|c|c|c|c|c|}
\hline \multirow{2}{*}{ No. } & \multirow{2}{*}{ LULC Type } & \multirow{2}{*}{ Actual LULC 2019} & \multicolumn{3}{|c|}{ Suitable LULC Allocation in $2049\left(\mathrm{~km}^{2}\right)$} \\
\hline & & & Dry Years & Normal Years & Wet Years \\
\hline 1 & Urban and built-up areas & 65.84 & 65.84 & 65.84 & 65.84 \\
\hline 2 & Paddy fields & 2012.16 & 1812.14 & 1812.13 & 1812.18 \\
\hline 3 & Sugarcane & 306.85 & 517.12 & 512.07 & 599.34 \\
\hline 4 & Cassava & 489.91 & 330.33 & 330.33 & 330.34 \\
\hline 5 & Other field crops & 6.19 & 6.19 & 11.27 & 11.25 \\
\hline 6 & Para rubber & 97.03 & 164.42 & 164.41 & 164.41 \\
\hline 7 & $\begin{array}{l}\text { Perennial trees and } \\
\text { orchards }\end{array}$ & 88.95 & 193.17 & 193.15 & 103.88 \\
\hline 8 & Forest land & 481.30 & 481.26 & 481.30 & 481.30 \\
\hline 9 & Waterbodies & 53.30 & 42.77 & 42.80 & 43.78 \\
\hline 10 & Rangeland & 71.65 & 66.07 & 66.04 & 66.01 \\
\hline 11 & Marshes and swamps & 27.73 & 21.57 & 21.58 & 22.56 \\
\hline 12 & Unused land & 93.32 & 93.30 & 93.28 & 93.28 \\
\hline & Total & 3794.22 & 3794.22 & 3794.22 & 3794.22 \\
\hline
\end{tabular}

Table 35. Present and future economic value of agricultural and forest land.

\begin{tabular}{|c|c|c|c|c|c|}
\hline LULC Types & $\begin{array}{c}\text { Price } \\
\text { (Baht/ton) }\end{array}$ & $\begin{array}{c}\text { Yield } \\
\text { (ton } / \mathbf{k m}^{2} \text { ) }\end{array}$ & $\begin{array}{l}\text { Present Value } \\
\left(\text { Baht } / \text { km }^{2}\right)\end{array}$ & $\begin{array}{c}\text { Discount Rate } \\
(\%)\end{array}$ & $\begin{array}{c}\text { Future Value in } \\
2049 \text { Using PV } \\
\text { Model } \\
\left(\text { Baht } / \mathrm{km}^{2}\right)\end{array}$ \\
\hline Paddy field ${ }^{1}$ & $13,287.75$ & 218.75 & $2,906,695.31$ & 6.50 & $19,225,947.12$ \\
\hline Sugarcane ${ }^{2}$ & 900.00 & 4468.75 & $4,021,875.00$ & 6.50 & $26,602,153.91$ \\
\hline Cassava ${ }^{1}$ & 1430.00 & 2240.63 & $3,204,093.75$ & 6.50 & $21,193,049.28$ \\
\hline Other field crops ${ }^{1}$ & 8092.50 & 415.63 & $3,363,445.31$ & 6.50 & $22,247,058.87$ \\
\hline Para rubber ${ }^{3}$ & $43,685.83$ & 131.25 & $5,733,765.63$ & 6.50 & $37,925,225.34$ \\
\hline Perennial trees and orchard ${ }^{1}$ & $25,600.00$ & 247.24 & $6,329,440.00$ & 6.50 & $41,865,233.77$ \\
\hline Forest land ${ }^{4}$ & - & - & $25,000,000.00$ & 6.50 & $165,359,154.08$ \\
\hline
\end{tabular}

Source: ${ }^{1}[72],{ }^{2}[73],{ }^{3}[74],{ }^{4}[75]$, and ${ }^{5}$ discount rate was based on the minimum retail rate of Bank for [76].

Table 36. Economic value by LULC types of actual LULC 2019 and suitable LULC allocation for flood mitigation in 2049.

\begin{tabular}{|c|c|c|c|c|}
\hline \multirow{3}{*}{ LULC Types } & \multicolumn{4}{|c|}{ Economic Value in 2049 (Baht) } \\
\hline & \multirow{2}{*}{ Actual LULC in 2019} & \multicolumn{3}{|c|}{ Suitable LULC Allocation for Flood Mitigation in 2049} \\
\hline & & Dry Year & Normal Year & Wet Year \\
\hline Paddy fields & $38,654.20$ & $34,629.20$ & $34,822.30$ & $34,826.09$ \\
\hline Sugarcane & 8147.42 & $13,678.87$ & $13,614.03$ & $15,920.81$ \\
\hline Cassava & $10,347.30$ & 6783.21 & 6979.08 & 6968.65 \\
\hline Other field crops & 137.81 & 136.47 & 200.36 & 200.68 \\
\hline Para rubber & 3675.10 & 6145.56 & 6235.43 & 6222.15 \\
\hline Perennial trees and orchards & 3717.46 & 9041.53 & 8055.82 & 4404.83 \\
\hline Forest land & $79,388.71$ & $78,164.79$ & $79,303.89$ & $79,315.05$ \\
\hline Total in million Baht & $144,068.00$ & $148,579.64$ & $149,210.92$ & $147,858.27$ \\
\hline
\end{tabular}

According to results, the top three dominant LULC types of actual LULC data in 2019 were paddy fields, cassava, and forest land, while the top three dominant LULC types of suitable LULC allocation data in 2049 for flood mitigation in dry, normal, and wet years were paddy field, sugarcane, and forest land. Moreover, the top three highest future values were forest land, perennial trees and orchards, and para rubber (Table 35).

As a result, in Table 36, the suitable LULC allocation data for flood mitigation in normal years provided the highest economic value, approximately 149,211 million Baht. In contrast, 
the actual LULC allocation in 2019 provided the lowest economic value, approximately 144,068 million Baht. The contribution of the economic value by LULC type from the actual LULC and suitable LULC allocation data for flood mitigation from three rainfall conditions are displayed in Figure 22.

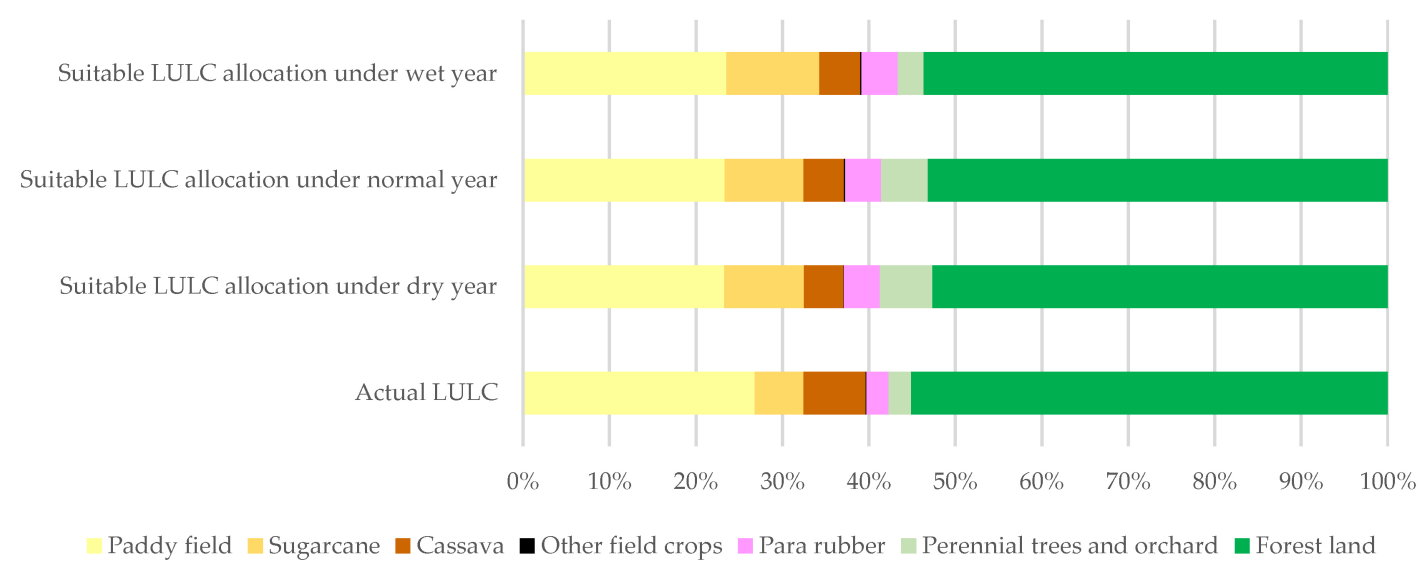

Figure 22. Contribution of the future economic value of LULC type of actual LULC and suitable LULC allocation for flood mitigation (dry, normal, and wet years).

According to the data in Figure 22, the top three dominant LULC types of actual LULC in 2019, including paddy fields, cassava, and forest land, will provide future economic value in 2049 , about $89 \%$ of the total value. Meanwhile, the top three dominant LULC types of suitable LULC allocation for flood mitigation under dry, normal, and wet years, including paddy fields, sugarcane, and forest land, deliver future economic value in 2049 of approximately $85 \%, 86 \%$, and $88 \%$ of the total value, respectively.

Moreover, the future economic value of forest land from actual LULC and suitable LULC allocation data for flood mitigation (dry, normal, and wet years) contributed the highest values compared to the other LULC types because the present economic value, approximately $25,000,000$ Baht per $\mathrm{km}^{2}$, or the future economic value, approximately $165,359,154$ Baht per $\mathrm{km}^{2}$, was exceptionally high when compared to other LULC types (see Table 35).

Furthermore, the results of the future economic value change by comparing the values of actual LULC data and each suitable LULC allocation for flood mitigation (dry, normal, and wet years) in terms of gain (+sign) and loss (-sign) are reported in Tables 37-39 and spatially displayed in Figure 23.

According to change detection, suitable LULC allocation for flood mitigation in 2049 in normal years gained the highest future economic value of approximately 4322 million Baht (Table 38). On the contrary, suitable LULC allocation for flood mitigation in 2049 in wet years gained the highest future economic value of approximately 3124 million Baht (Table 39). These results show the consequence of LULC allocation for flood mitigation in 2049 using goal programming on future economic value, because the future economic value depends on the areas of LULC type and their values. 
Table 37. Future economic value change in 2049 between actual LULC in 2019 and suitable LULC allocation in dry years.

\begin{tabular}{|c|c|c|c|c|c|c|c|c|c|}
\hline & \multirow{2}{*}{ LULC Types } & \multicolumn{7}{|c|}{ Suitable LULC Allocation in 2049 of Dry Years } & \multirow{2}{*}{ Total } \\
\hline & & PA & SU & CA & FC & PR & PO & FO & \\
\hline \multirow{7}{*}{ 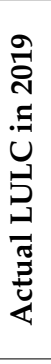 } & Paddy fields (PA) & 0 & 1507.30 & 28.21 & 0.02 & 18.11 & 602.05 & 1656.23 & 3811.92 \\
\hline & Sugarcane (SU) & -188.27 & 0 & -105.11 & -0.99 & 14.05 & 173.00 & 790.00 & 682.69 \\
\hline & Cassava (CA) & -28.21 & 207.96 & 0 & 0.28 & 813.50 & 1983.53 & 1249.23 & 4226.29 \\
\hline & Other field crops (FC) & -0.01 & 0.91 & -0.11 & 0 & 12.19 & 0.42 & 104.33 & 117.73 \\
\hline & Para rubber (PR) & 0 & -2.68 & -25.43 & -8.99 & 0 & 2.45 & 1167.21 & 1132.55 \\
\hline & $\begin{array}{l}\text { Perennial trees and } \\
\text { orchards (PO) }\end{array}$ & -193.53 & -123.84 & -86.03 & -0.35 & -5.10 & 0 & 237.52 & -171.32 \\
\hline & Forest land (FO) & -131.26 & $\begin{array}{c}- \\
2240.87\end{array}$ & -630.19 & -95.31 & -2467.68 & -305.09 & 0 & -5870.41 \\
\hline & Total & -541.27 & -651.22 & -818.66 & -105.34 & -1614.92 & 2456.36 & 5204.51 & 3929.45 \\
\hline
\end{tabular}

Table 38. Future economic value change in 2049 between actual LULC in 2019 and suitable LULC allocation in normal years.

\begin{tabular}{|c|c|c|c|c|c|c|c|c|c|}
\hline & \multirow{2}{*}{ LULC Types } & \multicolumn{7}{|c|}{ Suitable LULC Allocation in 2049 of Normal Years } & \multirow{2}{*}{ Total } \\
\hline & & PA & SU & CA & FC & PR & PO & FO & \\
\hline \multirow{8}{*}{ 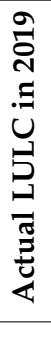 } & Paddy fields (PA) & 0 & 1453.48 & 28.90 & 0.02 & 38.03 & 563.56 & 1656.23 & 3740.22 \\
\hline & Sugarcane (SU) & -190.75 & 0 & -108.03 & -1.06 & 21.53 & 151.87 & 790.00 & 663.57 \\
\hline & Cassava (CA) & -29.13 & 224.39 & 0 & 0.59 & 975.32 & 1546.54 & 1252.86 & 3970.57 \\
\hline & Other field crops (FC) & -0.01 & 0.94 & -0.11 & 0 & 12.05 & 0.41 & 104.46 & 117.74 \\
\hline & Para rubber (PR) & 0 & -2.69 & -26.55 & -41.85 & 0 & 2.45 & 1206.43 & 1137.79 \\
\hline & $\begin{array}{l}\text { Perennial trees and } \\
\text { orchards (PO) }\end{array}$ & -196.66 & -123.25 & -88.76 & -0.64 & -6.05 & 0 & 237.74 & -177.62 \\
\hline & Forest land (FO) & -152.56 & -2237.12 & -645.50 & -142.20 & -1666.34 & -286.86 & 0 & -5130.59 \\
\hline & Total & -569.12 & -684.25 & -840.06 & -185.14 & -625.45 & 1977.96 & 5247.72 & 4321.67 \\
\hline
\end{tabular}

Table 39. Future economic value change in 2049 between actual LULC in 2019 and suitable LULC allocation in wet years.

\begin{tabular}{|c|c|c|c|c|c|c|c|c|c|}
\hline & \multirow{2}{*}{ LULC Types } & \multicolumn{7}{|c|}{ Suitable LULC Allocation in 2049 of the Wet Year } & \multirow{2}{*}{ Total } \\
\hline & & PA & SU & CA & FC & PR & PO & FO & \\
\hline \multirow{8}{*}{ 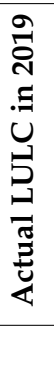 } & Paddy fields (PA) & 0 & 1525.95 & 27.55 & 0.02 & 58.57 & 323.42 & 1656.23 & 3591.74 \\
\hline & Sugarcane (SU) & -190.82 & 0 & -105.43 & -1.06 & 19.27 & 97.94 & 790.00 & 609.90 \\
\hline & Cassava (CA) & -29.16 & 565.99 & 0 & 0.59 & 971.75 & 245.81 & 1252.86 & 3007.84 \\
\hline & Other field crops (FC) & -0.01 & 0.95 & -0.12 & 0 & 11.92 & 0.35 & 104.46 & 117.55 \\
\hline & Para rubber (PR) & 0 & -2.69 & -31.35 & -41.89 & 0 & 2.45 & 1206.89 & 1133.40 \\
\hline & $\begin{array}{l}\text { Perennial trees and } \\
\text { orchards (PO) }\end{array}$ & -196.68 & -141.72 & -90.29 & -0.64 & -6.21 & 0 & 237.74 & -197.81 \\
\hline & Forest land (FO) & -152.83 & -2360.51 & -653.55 & -143.61 & -1569.08 & -259.19 & 0 & -5138.76 \\
\hline & Total & -569.50 & -412.03 & -853.19 & -186.59 & -513.78 & 410.78 & 5248.17 & 3123.86 \\
\hline
\end{tabular}




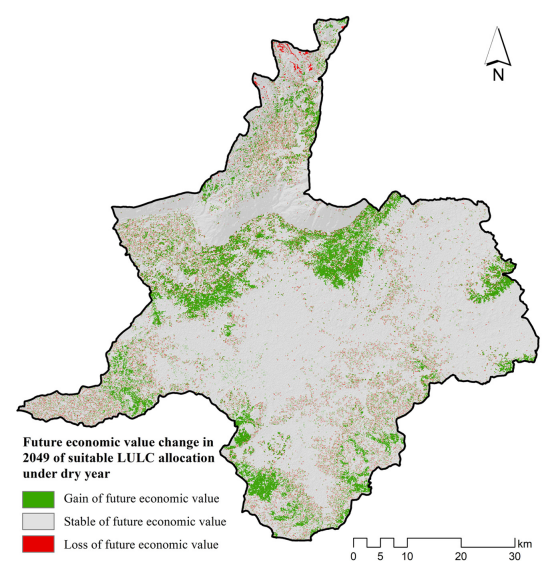

(a)

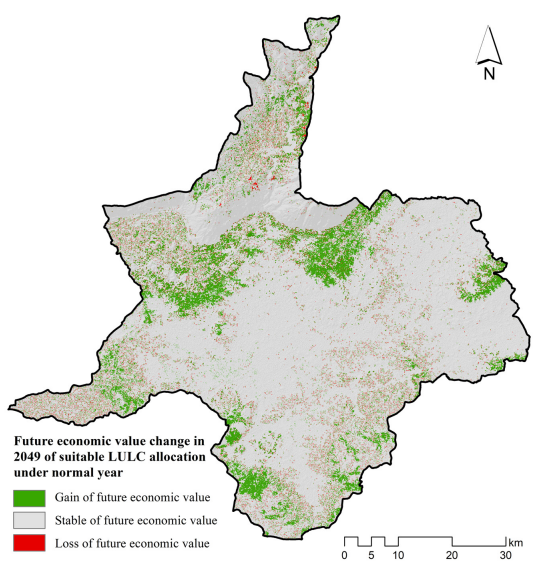

(b)

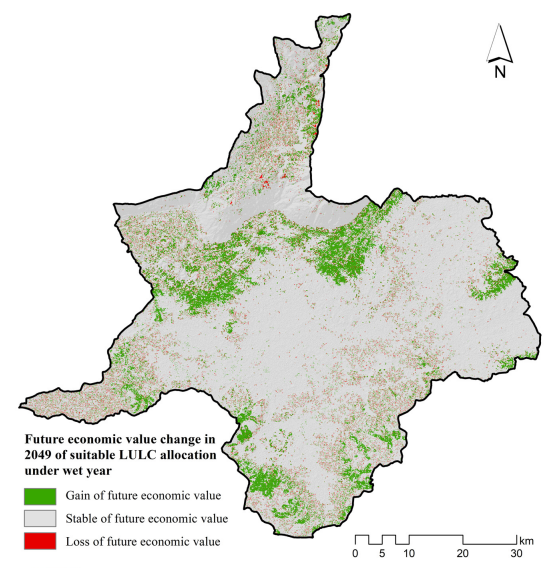

(c)

Figure 23. Gain and loss of future economic value of suitable LULC allocation for flood mitigation in 2049: (a) Dry years, (b) normal years, and (c) wet years.

\subsection{Ecosystem Service Value Evaluation and Change}

The actual LULC data in 2019 and the suitable LULC data in 2049 for flood mitigation under dry, normal, and wet years (Table 40) were applied to evaluate the ecosystem service values using a simple benefit transfer method according to a coefficient value of LULC type (see Table 4), and the results are shown in Table 41 and Figure 24.

Table 40. Area of each LULC type for ESV evaluation of actual LULC and suitable LULC allocation for flood mitigation under dry, normal, and wet years.

\begin{tabular}{clcccc}
\hline \multirow{2}{*}{ No. } & \multirow{2}{*}{ ESV-LULC Type } & Actual LULC & \multicolumn{3}{c}{ Suitable LULC Allocation in 2049 } \\
\cline { 3 - 6 } & $\mathbf{2 0 1 9}$ & Dry Years & Normal Years & Wet Years \\
\hline 1 & Urban and built-up areas & 65.84 & 65.84 & 65.84 & 65.84 \\
2 & Paddy fields & 2012.16 & 1812.16 & 1812.16 & 1812.16 \\
3 & Field crop & 802.95 & 853.64 & 853.64 & 940.94 \\
4 & Forest land & 667.28 & 838.84 & 838.84 & 749.54 \\
5 & Waterbodies & 53.3 & 42.78 & 42.78 & 63.78 \\
6 & Rangeland & 71.65 & 66.05 & 66.05 & 66.05 \\
7 & Marshes and swamps & 27.73 & 21.61 & 21.61 & 22.61 \\
8 & Unused land & 93.32 & 93.31 & 93.31 & $\mathbf{3 7 9 4 . 2 2}$ \\
\hline
\end{tabular}

Table 41. Ecosystem service value by ESV-LULC types of actual LULC 2019 and suitable LULC allocation for flood mitigation in 2049.

\begin{tabular}{|c|c|c|c|c|}
\hline \multirow{3}{*}{ ESV-LULC Types } & \multicolumn{4}{|c|}{ Ecosystem Service Value (Baht) } \\
\hline & \multirow{2}{*}{ Actual LULC in 2019} & \multicolumn{3}{|c|}{ Suitable LULC Allocation for Flood Mitigation in 2049} \\
\hline & & Dry Years & Normal Years & Wet Years \\
\hline Urban and built-up areas (UR) & 2.60 & 2.62 & 2.62 & 2.62 \\
\hline Paddy fields (PA) & 6478.73 & 5804.11 & 5836.47 & 5837.11 \\
\hline Field crops (FC) & 2580.20 & 2708.13 & 2739.31 & 3017.20 \\
\hline Forest land (FO) & 4050.69 & 5175.68 & 5088.76 & 4556.47 \\
\hline Waterbodies (WA) & 1143.07 & 1019.07 & 923.23 & 951.98 \\
\hline Rangeland (RA) & 180.65 & 176.46 & 167.47 & 163.55 \\
\hline Marshes and swamps (MA) & 810.57 & 567.00 & 642.80 & 679.99 \\
\hline Unused land (UL) & 27.92 & 27.94 & 27.94 & 27.94 \\
\hline Total in million Baht & $15,274.42$ & $15,481.00$ & $15,428.61$ & $15,236.86$ \\
\hline
\end{tabular}




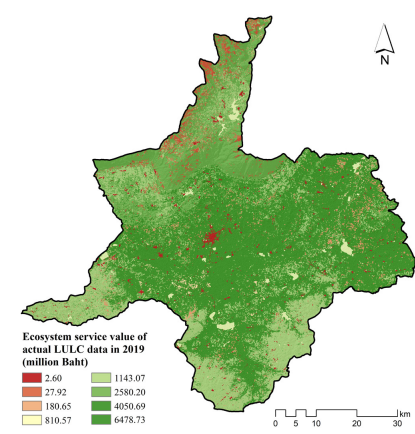

(a)

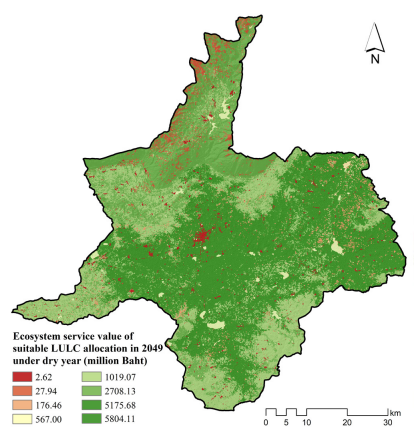

(b)

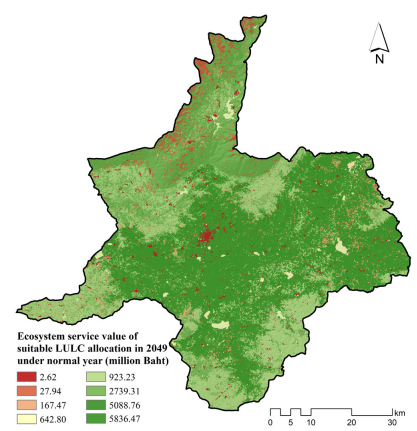

(c)

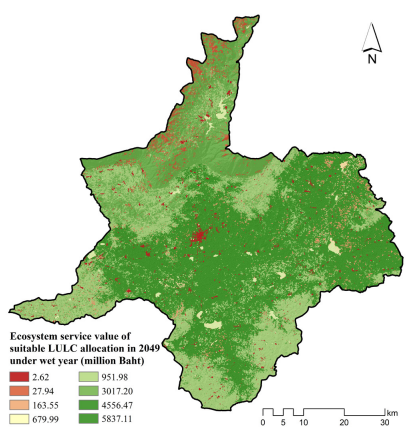

(d)

Figure 24. Spatial distribution of the ecosystem service value: (a) Actual LULC 2019, (b) dry years, (c) normal years, and (d) wet years.

As a result, the ESV of actual LULC in 2019 and the suitable LULC allocation for flood mitigation in 2049 in dry, normal, and wet years are slightly different. The suitable LULC allocation for flood mitigation in 2049 under the dry year provided the highest ESV, approximately 15,481 million Baht, while the suitable LULC allocation for flood mitigation in 2049 in wet years delivered the lowest ESV, approximately 15,237 million Baht. The contributions of ESV of each LULC type from actual LULC in 2019 and the suitable LULC allocation for flood mitigation in 2049 under the three rainfall conditions are compared in Figure 25.

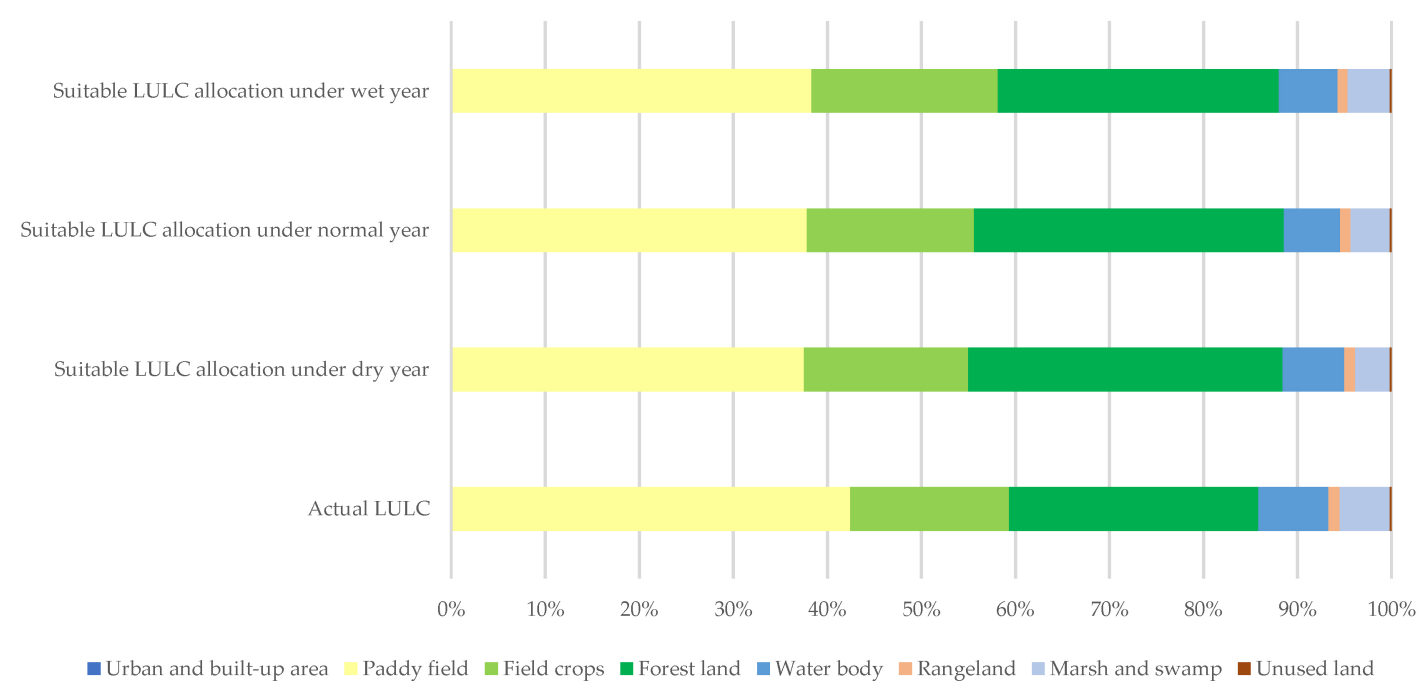

Figure 25. Contribution of the ecosystem service value of each LULC type of actual LULC and suitable LULC allocation for flood mitigation (dry, normal, and wet years).

As shown in Figure 25, paddy fields provided the highest ESV value from the actual LULC and the suitable LULC allocation data for flood mitigation in 2049 under the three rainfall conditions, because paddy fields were the most dominant type in the area. Additionally, the top three dominant LULC types, namely, paddy fields, field crops, and forest land, delivered ESVs of approximately $86 \%, 88 \%, 89 \%$, and $88 \%$ of the total value.

Furthermore, the results of the ESV change upon comparison between values of actual LULC data and each suitable LULC allocation for flood mitigation (dry, normal, and wet years) in terms of gain (+sign) and loss (-sign) are reported in Tables 42-44 and spatially displayed in Figure 26. 
Table 42. Ecosystem service value change between actual LULC in 2019 and suitable LULC allocation in 2049 in dry years.

\begin{tabular}{|c|c|c|c|c|c|c|c|c|c|c|}
\hline & \multirow{2}{*}{ LULC Types } & \multicolumn{8}{|c|}{ Suitable LULC Allocation in 2049 of Dry Years } & \multirow{2}{*}{ Total } \\
\hline & & UR & PA & FC & FO & WA & RA & MA & UL & \\
\hline \multirow{8}{*}{ 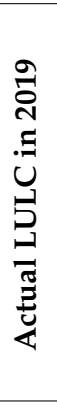 } & $\begin{array}{l}\text { Urban and built-up areas } \\
\text { (UR) }\end{array}$ & 0 & 33.07 & 14.03 & 14.06 & 4.88 & 0.17 & 2.05 & 0.08 & 68.34 \\
\hline & Paddy fields (PA) & -36.36 & 0 & 0 & 111.30 & 40.04 & -10.79 & 119.19 & -1.86 & 221.52 \\
\hline & Field crops (FC) & -14.69 & 0 & 0 & 495.14 & 16.80 & -3.06 & 5.11 & -18.86 & 480.44 \\
\hline & Forest land (FO) & -9.60 & -27.03 & -102.47 & 0 & 13.13 & -3.09 & 0.65 & -91.86 & -220.28 \\
\hline & Waterbodies (WA) & -4.93 & -46.67 & -15.46 & -20.49 & 0 & -0.14 & 39.86 & -0.82 & -48.65 \\
\hline & Rangeland (RA) & -0.16 & 8.88 & 4.30 & 12.49 & 0.19 & 0 & 0.75 & -0.02 & 26.42 \\
\hline & $\begin{array}{l}\text { Marshes and swamps } \\
\text { (MA) }\end{array}$ & -1.97 & -184.81 & -6.07 & -249.41 & -0.91 & -1.64 & 0 & 0 & -444.81 \\
\hline & Unused land (UL) & -0.07 & 1.28 & 8.82 & 112.93 & 0.63 & 0.01 & 0 & 0 & 123.60 \\
\hline & Total & -67.79 & -215.28 & -96.84 & 476.01 & 74.76 & -18.53 & 167.60 & -113.35 & 206.58 \\
\hline
\end{tabular}

Table 43. Ecosystem service value change between the actual LULC in 2019 and the suitable LULC allocation in 2049 in normal years.

\begin{tabular}{|c|c|c|c|c|c|c|c|c|c|c|}
\hline & \multirow{2}{*}{ LULC Types } & \multicolumn{8}{|c|}{ Suitable LULC Allocation in 2049 of Normal Years } & \multirow{2}{*}{ Total } \\
\hline & & UR & PA & FC & FO & WA & RA & MA & UL & \\
\hline \multirow{9}{*}{ 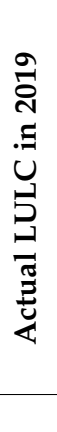 } & $\begin{array}{l}\text { Urban and built-up areas } \\
\text { (UR) }\end{array}$ & 0 & 33.21 & 14.25 & 13.47 & 4.68 & 0.15 & 2.18 & 0.08 & 68.02 \\
\hline & Paddy fields (PA) & -36.36 & 0 & 0 & 109.49 & 31.33 & -10.30 & 119.54 & -1.86 & 211.83 \\
\hline & Field crops (FC) & -14.69 & 0 & 0 & 460.30 & 16.74 & -2.72 & 4.87 & -18.86 & 445.63 \\
\hline & Forest land (FO) & -9.60 & -27.85 & -110.14 & 0 & 13.13 & -2.75 & 0.65 & -91.86 & -228.41 \\
\hline & Waterbodies (WA) & -4.93 & -46.67 & -16.38 & -19.96 & 0 & -0.14 & 70.47 & -0.82 & -18.44 \\
\hline & Rangeland (RA) & -0.16 & 8.98 & 4.27 & 20.19 & 0.15 & 0 & 0.77 & -0.02 & 34.19 \\
\hline & $\begin{array}{l}\text { Marshes and swamps } \\
\text { (MA) }\end{array}$ & -1.97 & -184.81 & -54.01 & -238.61 & -0.69 & -1.64 & 0 & 0 & -481.73 \\
\hline & Unused land (UL) & -0.07 & 1.32 & 9.31 & 111.90 & 0.63 & 0.01 & 0 & 0 & 123.09 \\
\hline & Total & -67.79 & -215.81 & -152.70 & 456.77 & 65.98 & -17.39 & 198.47 & -113.35 & 154.18 \\
\hline
\end{tabular}

Table 44. Ecosystem service value change between the actual LULC in 2019 and the suitable LULC allocation in 2049 in wet years.

\begin{tabular}{|c|c|c|c|c|c|c|c|c|c|c|}
\hline & \multirow{2}{*}{ LULC Types } & \multicolumn{8}{|c|}{ Suitable LULC Allocation in 2049 of Wet Years } & \multirow{2}{*}{ Total } \\
\hline & & UR & PA & FC & FO & WA & RA & MA & UL & \\
\hline \multirow{8}{*}{ 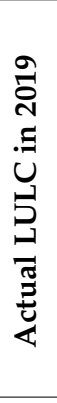 } & $\begin{array}{l}\text { Urban and built-up areas } \\
\text { (UR) }\end{array}$ & 0 & 33.22 & 15.59 & 10.91 & 4.76 & 0.14 & 2.10 & 0.08 & 66.81 \\
\hline & Paddy fields (PA) & -36.36 & 0 & 0 & 82.27 & 34.28 & -10.04 & 130.26 & -1.86 & 198.55 \\
\hline & Field crops (FC) & -14.69 & 0 & 0 & 268.93 & 16.79 & -2.64 & 3.65 & -18.86 & 253.17 \\
\hline & Forest land (FO) & -9.60 & -27.85 & -117.37 & 0 & 13.13 & -2.62 & 0.85 & -91.86 & -235.33 \\
\hline & Waterbodies (WA) & -4.93 & -46.67 & -19.20 & -17.57 & 0 & -0.14 & 61.42 & -0.82 & -27.92 \\
\hline & Rangeland (RA) & -0.16 & 8.99 & 4.68 & 21.78 & 0.15 & 0 & 0.72 & -0.02 & 36.14 \\
\hline & Marshes and swamps & -1.97 & -184.81 & -214.16 & -48.07 & -0.76 & -1.64 & 0 & 0 & -451.40 \\
\hline & Unused land (UL) & -0.07 & 1.32 & 10.00 & 110.52 & 0.63 & 0.01 & 0 & 0 & 122.41 \\
\hline & Total & -67.79 & -215.81 & -320.47 & 428.78 & 68.99 & -16.92 & 199.01 & -113.35 & -37.56 \\
\hline
\end{tabular}




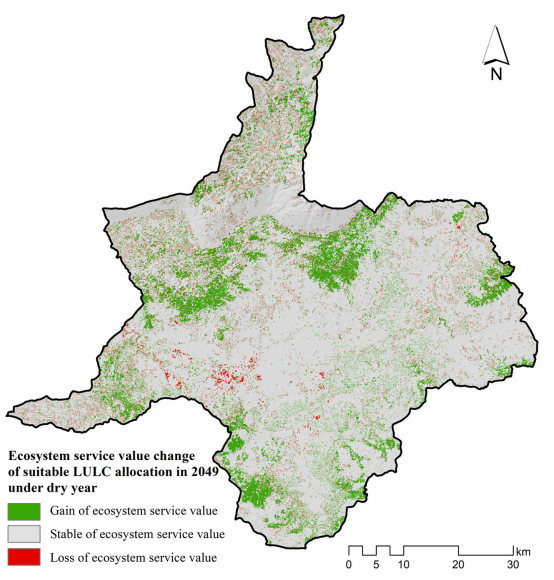

(a)

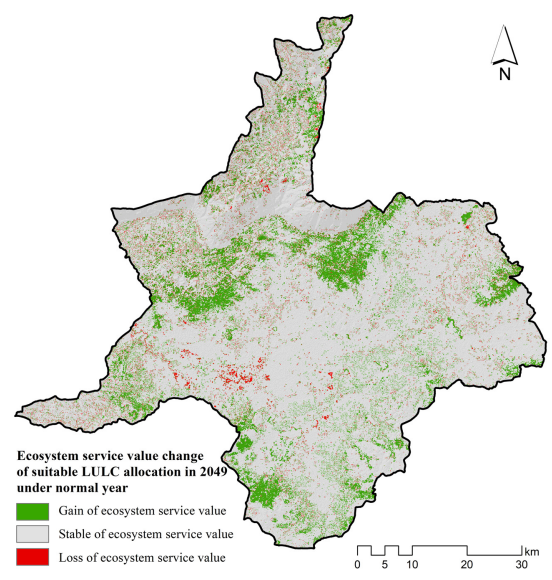

(b)

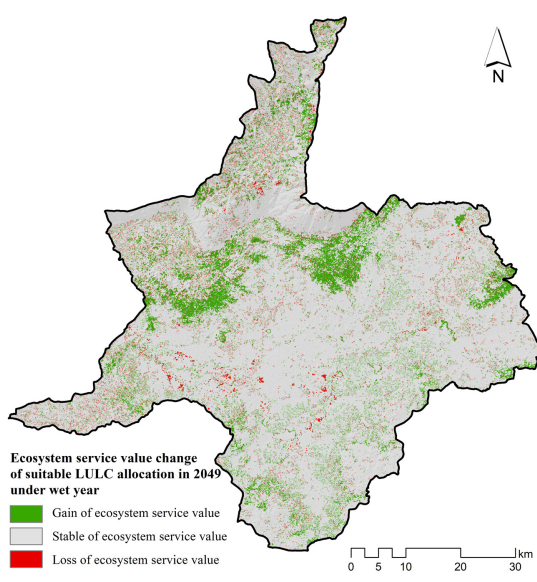

(c)

Figure 26. Gain and loss of ESV of suitable LULC allocation for flood mitigation in 2049: (a) Dry years, (b) normal years, and (c) wet years.

According to the results in Tables 42-44, the suitable LULC allocation for flood mitigation in 2049 under dry years gained the highest ESV of approximately 207 million Baht. On the contrary, the suitable LULC allocation for flood mitigation in 2049 in wet years resulted in losses in ESV of approximately 38 million Baht. These findings indicate that the ESV of suitable LULC allocation for flood mitigation in 2049 in wet years is lower than the actual LULC in 2019 (see Table 45). Similar to the change in future economic value, these results show the consequence of LULC allocation for flood mitigation in 2049 using goal programming on ESV, because the ESV depends on areas of LULC type and their coefficient values. Moreover, it can be observed that forest land, waterbodies, and marshes and swamps provide a gain in ESV (+sign), while urban and built-up areas, paddy fields, field crops, rangeland, and unused land results in a loss of ESV (-sign) for all three rainfall conditions. These findings indicate that the ecosystem service value was dictated by the coefficient value of each LULC type.

Table 45. Future economic and ESV value evaluation and change and reduction in surface runoff of each suitable LULC allocation for flood mitigation by comparison to the baseline information of LULC data in 2019.

\begin{tabular}{lccc}
\hline \multirow{2}{*}{ Item } & \multicolumn{2}{c}{ Suitable LULC Allocation for Flood Mitigation in 2049 } \\
\cline { 2 - 3 } & Dry Years & Normal Years & Wet Years \\
\hline Future economic value (million Baht) & $148,579.64$ & $149,210.92$ & $147,858.27$ \\
Gain or loss by economic value (million Baht) & 3929.45 & 4321.67 & 3123.86 \\
Ecosystem service value (million Baht) & $15,481.00$ & $15,428.61$ & $15,236.86$ \\
Gain or loss by ESV (million Baht) & 206.58 & 154.18 & -37.56 \\
Runoff reduction (million m $^{3}$ ) & 27.89 & 52.31 & 46.73 \\
\hline
\end{tabular}

In summary, the suitable LULC allocation for flood mitigation in 2049 in normal years provides the highest value for future economic value evaluation and the highest gain value compared to actual LULC in 2019. In the meantime, the suitable LULC allocation for flood mitigation in 2049 in dry years provided the highest value for ecosystem service evaluation and the highest gain the ESV by comparing it with actual LULC in 2019. Meanwhile, the suitable LULC allocation for flood mitigation in 2049 in normal years can reduce the highest surface runoff by approximately 52 million $\mathrm{m}^{3}$ compared to the actual LULC in 2019 (see Table 45).

Consequently, it can be concluded that the most suitable LULC allocation for flood mitigation in 2049 in Chaiyaphum district, Chaiyaphum province under the Second Part of the Lam Nam Chi watershed, based on the future economic value and ecosystem service value evaluation, is a suitable LULC allocation for flood mitigation in 2049 in the 
normal year scenario. This information can be used as primary data for supporting project implementation.

\section{Conclusions}

This study applied the supervised method to classify LULC data in 2001, 2010, and 2019 based on Landsat 5-TM and Landsat 8-OLI with supplementary data, including NDVI, MNDWI, NDBI, and DEM, using an RF classifier under EnMap BOX software. The derived thematic accuracy of the LULC maps showed an overall accuracy and Kappa hat coefficient between classified LULC maps and ground reference data in 2001, 2010, and 2019 of 89.88\% and $84.88 \%, 90.71 \%$ and $87.03 \%$, and $91.37 \%$ and $88.26 \%$, respectively. Later, the classified LULC data in 2001, 2010, and 2019 were further applied to predict the LULC change in two periods, 2002-2009 and 2011-2018, using the CLUE-S model. The significant driving factors of LULC change for specific LULC type location preferences included elevation, slope, annual rainfall, average income per capita at the sub-district level, population density at the sub-district level, distance to the road network, distance to a stream, and distance to the existing urban area. As a result, the LULC prediction of both periods was dictated by the historical LULC development between 2001 and 2010 and 2010 and 2019, respectively. Then, time series surface runoff data between 2001 and 2019 were estimated using the SCS-CN method under a GIS raster-based environment. The process worked on spatial variation of land use, hydrologic soil group, and rainfall data. In this study, a suitable AMC condition was first examined and validated for time series surface runoff estimation between 2001 and 2010. Then, a suitable AMC condition was further chosen to estimate the time series surface runoff between 2011 and 2019.

After this, goal programming was applied to minimize the surface runoff for flood mitigation based on the surface runoff coefficient value of LULC types in dry, normal, and wet years for 2029, 2039, and 2049. Accordingly, the surface runoff could be reduced under all three rainfall conditions, and suitable LULC allocation for flood mitigation in dry, normal, and wet years was in 2049. The suitable LULC allocation for flood mitigation in 2049 of the normal year provided the highest value and gain for future economic value compared to the actual LULC in 2019. Meanwhile, the suitable LULC allocation for flood mitigation in 2049 of dry years provided the highest value and gain for ecosystem services compared to the actual LULC in 2019. Nonetheless, considering the future economic and ecosystem service values and changes in surface runoff reduction, the most suitable LULC allocation for flood mitigation in 2049 was normal years.

In conclusion, the derived results of this study can be used as primary information for flood mitigation project implementation in Chaiyaphum province. Likewise, the presented conceptual framework and research workflows can be used as a guideline for government agencies to examine flood-prone areas for flood mitigation in Thailand.

However, to apply the proposed method in other areas, we recommend that the $\mathrm{CN}$ value of the AMC-II condition, as the identified suitable value in this study, can directly apply to estimate time series surface runoff. In addition, rainfall conditions identification using SPI can be ignored to increase the number of years for calculating the average runoff coefficient value of each LULC type. This value plays a vital role in minimizing surface runoff for flood mitigation using goal programming.

Author Contributions: Conceptualization, S.O. and A.P.; methodology, S.O. and A.P.; software, A.P.; validation, S.O. and A.P.; formal analysis, S.O. and A.P.; investigation, S.O. and A.P.; data curation, A.P.; writing—original draft preparation, A.P.; writing—review and editing, S.O.; visualization, A.P.; supervision, S.O. All authors have read and agreed to the published version of the manuscript.

Funding: This research received no external funding.

Institutional Review Board Statement: Not applicable.

Informed Consent Statement: Not applicable.

Data Availability Statement: Not applicable. 
Acknowledgments: The authors would like to thank the Suranaree University of Technology for supporting the facilities to undertake this research. Additionally, the special thanks from the authors go to anonymous reviewers for their valuable comments and suggestions that improve our manuscript from various perspectives.

Conflicts of Interest: The authors declare no conflict of interest.

\section{References}

1. Yu, D.; Xie, P.; Dong, X.; Hu, B.; Ji, L.; Li, Y.; Peng, T.; Ma, H.; Wang, K.; Xu, S. Improvement of the SWAT model for event-based flood simulation on a sub-daily timescale. Hydrol. Earth Syst. Sci. Discuss. 2018, 22, 5001-5019. [CrossRef]

2. Jothityangkoon, C.; Maskong, H.; Sangthong, P.; Kosa, P. Development processes of a master plan for flood protection and mitigation in a community area: A case study of Roi Et province. KKU Eng. J. 2015, 42, 287-291.

3. Kuntiyawichai, K.; Sri-Amporn, W.; Wongsasri, S.; Chindaprasirt, P. Anticipating of potential climate and land use change impacts on floods: A case study of the lower Nam Phong river basin. Water 2020, 12, 1158. [CrossRef]

4. Mbow, C.; Diop, A.; Diaw, A.T.; Niang, C.I. Urban sprawl development and flooding at Yeumbeul suburb (Dakar-Senegal). Afr. J. Environ. Sci. Technol. 2008, 2, 75-88.

5. Prasad, A.S.; Pandey, B.W.; Leimgruber, W.; Kunwar, R.M. Mountain hazard susceptibility and livelihood security in the upper catchment area of the river Beas, Kullu Valley, Himachal Pradesh, India. Geoenvironmental Disasters 2016, 3, 1-17. [CrossRef]

6. Billi, P.; Alemu, Y.T.; Ciampalini, R. Increased frequency of flash floods in Dire Dawa, Ethiopia: Change in rainfall intensity or human impact? Nat. Hazards 2015, 76, 1373-1394. [CrossRef]

7. Danumah, J.H.; Odai, S.N.; Saley, B.M.; Szarzynski, J.; Thiel, M.; Kwaku, A.; Kouame, F.K.; Akpa, L.Y. Flood risk assessment and mapping in Abidjan district using multi-criteria analysis (AHP) model and geoinformation techniques, (cote d'ivoire). Geoenvironmental Disasters 2016, 3, 1-13. [CrossRef]

8. Department of Disaster Prevention and Mitigation. Report of Damage from a Flooding Situation; Ministry of Interior: Bangkok, Thailand, 2019.

9. Sriwongsitanon, N. Flood forecasting system development for the upper Ping River basin. Kasetsart J. Nat. Sci. 2010, 44, 717-731.

10. Banba, M. Influences of regional development on land use of Nagara Basin and flood risk control. In Proceedings of the 3rd European Conference on Flood Risk Management, Lyon, France, 17-21 October 2016; pp. 1-7.

11. Tajbakhsh, S.M.; Memarian, H.; Kheyrkhah, A. A GIS-based integrative approach for land use optimization in a semi-arid watershed. Glob. J. Environ. Sci. Manag. 2018, 4, 31-46.

12. Garg, V.; Nikam, B.R.; Thakur, P.K.; Aggarwal, S.P.; Gupta, P.K.; Srivastav, S.K. Human-induced land use land cover change and its impact on hydrology. HydroResearch 2019, 1, 48-56. [CrossRef]

13. Leta, M.K.; Demissie, T.A.; Tränckner, J. Hydrological responses of watershed to historical and future land use land cover change dynamics of Nashe watershed, Ethiopia. Water 2021, 13, 2372. [CrossRef]

14. Kuntiyawichai, K. Interactions between Land Use and Flood Management in the Chi River Basin; Wageningen University: Wageningen, The Netherlands, 2012.

15. Tingsanchali, T.; Karim, F. Flood-hazard assessment and risk-based zoning of a tropical flood plain: Case study of the Yom River, Thailand. Hydrol. Sci. J. 2010, 55, 145-161. [CrossRef]

16. Riedel, C. Optimizing land use planning for mountainous regions using LP and GIS towards sustainability. India. J. Soil Conserv. 2003, 34, 121-124.

17. Sadeghi, S.H.R.; Jalili, K.; Nikkami, D. Land use optimization in watershed scale. Land Use Policy 2009, 26, 186-193. [CrossRef]

18. Nikkami, D.; Elektorowicz, M.; Mehuys, G.R. Optimizing the management of soil erosion. Water Pollut. Res. J. Can. 2002, 37, 577-586. [CrossRef]

19. Yeo, I.-Y.; Gordon, S.I.; Guldmann, J.-M. Optimizing patterns of land use to reduce peak runoff flow and nonpoint source pollution with an integrated hydrological and land use model. Earth Interact. 2004, 8, 1-20. [CrossRef]

20. Owji, M.R.; Nikkami, D.; Mahdian, M.H.; Mahmoudi, S. Minimizing surface runoff by optimizing land use management. World Appl. Sci. J. 2012, 20, 170-176.

21. Aldea, J.; Martínez-Peña, F.; Romero, C.; Diaz-Balteiro, L. Participatory goal programming in forest management: An application integrating several ecosystem services. Forests 2014, 5, 3352-3371. [CrossRef]

22. Gonfa, Z.B.; Kumar, D. Optimal land use planning in mojo watershed with multi-objective linear programming. Am. Int. J. Res. Hum. Arts Soc. Sci. 2015, 13, 10-17.

23. Al-Zahrani, M.; Musa, A.; Chowdhury, S. Multi-objective optimization model for water resource management: A case study for Riyadh, Saudi Arabia. Environ. Dev. Sustain. 2016, 18, 777-798. [CrossRef]

24. Sokouti, R.; Nikkami, D. Optimizing land use pattern to reduce soil erosion. Eurasian J. Soil Sci. 2017, 6, 75-83. [CrossRef]

25. Mellaku, M.T.; Reynolds, T.W.; Woldeamanuel, T. Linear programming-based cropland allocation to enhance performance of smallholder crop production: A pilot study in Abaro Kebele, Ethiopia. Resources 2018, 7, 76. [CrossRef]

26. Han, D.; Qiao, R.; Ma, X. Optimization of land-use structure based on the trade-off between carbon emission targets and economic development in Shenzhen, China. Sustainability 2019, 11, 11. [CrossRef]

27. Land Development Department. Soil Series of Thailand; Ministry of Agriculture and Cooperatives: Bangkok, Thailand, 2019. 
28. Land Development Department. Land use data of Thailand; Land Development Department, Ministry of Agriculture and Cooperatives: Bangkok, Thailand, 2016.

29. Rouse, J.; Haas, R.H.; Schell, J.A.; Deering, D. Monitoring vegetation systems in the great plains with ERTS. In Proceedings of the Third Earth Resources Technology Satellite-1 Symposium- Volume I: Technical Presentations. NASA SP-351, Greenbelt, MD, USA, 1 January 1973; pp. 3010-3017.

30. $\mathrm{Xu}, \mathrm{H}$. A new index for delineating built-up land features in satellite imagery. Int. J. Remote Sens. 2008, 29, 4269-4276. [CrossRef]

31. Zha, Y.; Gao, J.; Ni, S. Use of normalized difference built-up index in automatically mapping urban areas from TM imagery. Int. J. Remote Sens. 2003, 24, 583-594. [CrossRef]

32. Congalton, R.G.; Green, K. Assessing the Accuracy of Remotely Sensed Data: Principles and Practices, 2nd ed.; CRC Press: Boca Raton, FL, USA, 2019.

33. Trisurat, Y.; Alkemade, R.; Verburg, P.H. Projecting land-use change and its consequences for biodiversity in northern Thailand. Environ. Manag. 2010, 45, 626-639. [CrossRef] [PubMed]

34. Han, H.; Yang, C.; Song, J. Scenario simulation and the prediction of land use and land cover change in Beijing, China. Sustainability 2015, 7, 4260-4279. [CrossRef]

35. Ongsomwang, S.; Iamchuen, N. Integration of geospatial models for optimum land use allocation in three different scenarios. Suranaree J. Sci. Technol. 2015, 22, 377-396.

36. Zheng, H.W.; Shen, G.Q.; Wang, H.; Hong, J. Simulating land use change in urban renewal areas: A case study in Hong Kong. Habitat Int. 2015, 46, 23-34. [CrossRef]

37. Xu, X.; Du, Z.; Zhang, H. Integrating the system dynamic and cellular automata models to predict land use and land cover change. Int. J. Appl. Earth Obs. Geoinf. 2016, 52, 568-579. [CrossRef]

38. Gao, C.; Zhou, P.; Jia, P.; Liu, Z.; Wei, L.; Tian, H. Spatial driving forces of dominant land use/land cover transformations in the Dongjiang River watershed, Southern China. Environ. Monit. Assess. 2016, 188, 84. [CrossRef]

39. Ongsomwang, S.; Boonchoo, K. Integration of geospatial models for the allocation of deforestation hotspots and forest protection units. Suranaree J. Sci. Technol. 2016, 23, 283-307.

40. Li, X.; Wang, Y.; Li, J.; Lei, B. Physical and socioeconomic driving forces of land-use and land-cover changes: A case study of Wuhan City, China. Discrete Dyn. Nat. Soc. 2016, 2016, 1-11. [CrossRef]

41. Phompila, C.; Lewis, M.; Ostendorf, B.; Clarke, K. Forest cover changes in Lao tropical forests: Physical and socio-economic factors are the most important drivers. Land 2017, 6, 23. [CrossRef]

42. Arowolo, A.O.; Deng, X. Land use/land cover change and statistical modelling of cultivated land change drivers in Nigeria. Reg. Environ. Change 2018, 18, 247-259. [CrossRef]

43. Palchowdhuri, Y.; Roy, P.S. Driver based statistical model for simulating land use/land cover change in Indus river basin, India. Remote Sens. Land 2018, 2, 15-30. [CrossRef]

44. Ongsomwang, S.; Pattanakiat, S.; Srisuwan, A. Impact of land use and land cover change on ecosystem service values: A case study of Khon Kaen City, Thailand. Environ. Nat. Resour. J. 2019, 17, 43-58. [CrossRef]

45. Nguyen, H.H.; Dargusch, P.; Moss, P.; Aziz, A.A. Land-use change and socio-ecological drivers of wetland conversion in Ha Tien Plain, Mekong Delta, Vietnam. Land Use Policy 2017, 64, 101-113. [CrossRef]

46. Verburg, P.H.; Lesschen, J.-P. Practical: Explorative Modeling of Future Land Use for the Randstad Region of the Netherlands; Wageningen University: Wageningen, The Netherlands, 2014.

47. Chow, V.T.; Maidment, D.R.; Mays, L.W. Applied Hydrology; McGraw-Hill: New York, NY, USA, 1988.

48. United States Department of Agriculture. Urban Hydrology for Small Watersheds, Title 210-VI-TR-55, 2nd ed.; Conservation Engineering Division, National Resources Conservation Service, United States Department of Agriculture: Washington, DC, USA, 1986.

49. Weng, Q. Remote Sensing and GIS Integration Theories, Methods, and Applications; McGraw-Hill: New York, NY, USA, 2010.

50. Me, W.; Abell, J.M.; Hamilton, D.P. Effects of hydrologic conditions on SWAT model performance and parameter sensitivity for a small, mixed land use catchment in New Zealand. Hydrol. Earth Syst. Sci. 2015, 19, 4127-4147. [CrossRef]

51. Liu, Y.; Zhou, Y.; Ju, W.; Wang, S.; Wu, X.; He, M.; Zhu, G. Impacts of droughts on carbon sequestration by China' s terrestrial ecosystems from 2000 to 2011. Biogeosciences 2014, 11, 2583-2599. [CrossRef]

52. Rossiter, D.G. Lecture Notes: "Land Evaluation", Part 4: Economic Land Evaluation; College of Agriculture \& Life Sciences, Department of Soil, Crop, \& Atmospheric Sciences: Cornell University: Ithaca, NY, USA, 1994.

53. Costanza, R.; d'Arge, R.; de Groot, R.; Farber, S.; Grasso, M.; Hannon, B.; Limburg, K.; Naeem, S.; O’Neill, R.V.; Paruelo, J.; et al. The value of the world's ecosystem services and natural capital. Nature 1997, 387, 253-260. [CrossRef]

54. Millennium Ecosystem Assessment. In Ecosystems and Human Well-Being: Synthesis; Island Press: Washington, DC, USA, 2005.

55. TEEB. The Economics of Ecosystems and Biodiversity Ecological and Economic Foundations; Kumar, P., Ed.; Earthscan Publications: London, UK, 2010.

56. Mamat, A.; Halik, Ü.; Rouzi, A. Variations of ecosystem service value in response to land-use change in the Kashgar Region, Northwest China. Sustainability 2018, 10, 200. [CrossRef]

57. Anderson, J.R.; Hardy, E.E.; Roach, J.T.; Witmer, R.E. A Land Use and Land Cover Classification System for Use with Remote Sensor Data; 964; United States Government Printing Office: Washington, DC, USA, 1976; pp. 1-28. 
58. Fitzpatrick-Lins, K. Comparison of sampling procedures and data analysis for a land-use and land-cover map. Photogramm. Eng. Remote Sens. 1981, 47, 343-351.

59. Na, X.; Zhang, S.; Li, X.; Yu, H.; Liu, C. Improved land cover mapping using random forests combined with landsat thematic mapper imagery and ancillary geographic data. Photogramm. Eng. Remote Sens. 2010, 76, 833-840. [CrossRef]

60. Rodriguez-Galiano, V.F.; Ghimire, B.; Rogan, J.; Chica-Olmo, M.; Rigol-Sanchez, J.P. An assessment of the effectiveness of a random forest classifier for land-cover classification. ISPRS J. Photogramm. Remote Sens. 2012, 67, 93-104. [CrossRef]

61. Gartzia, M.; Alados, C.L.; Pe'rez-Cabello, F.; Bueno, C.G. Improving the accuracy of vegetation classifications in mountainous areas: A case study in the Spanish Central Pyrenees. Mt. Res. Dev. 2013, 33, 63-74. [CrossRef]

62. Jhonnerie, R.; Siregar, V.P.; Nababan, B.; Prasetyo, L.B.; Wouthuyzen, S. Random Forest Classification for mangrove land cover mapping using Landsat 5 TM and Alos Palsar imageries. Procedia Environ. Sci. 2015, 24, 215-221. [CrossRef]

63. Eisavi, V.; Homayouni, S.; Yazdi, A.M.; Alimohammadi, A. Land cover mapping based on random forest classification of multitemporal spectral and thermal images. Environ. Monit. Assess. 2015, 187, 291. [CrossRef] [PubMed]

64. Kulkarni, A.; Lowe, B. Random Forest algorithm for land cover classification. Int. J. Recent Innov. Trends Comput. Commun. 2016, 4, 58-63.

65. Pareeth, S.; Karimi, P.; Shafiei, M.; De Fraiture, C. Mapping agricultural landuse patterns from time series of Landsat 8 using random forest based hierarchical approach. Remote Sens. 2019, 11, 601. [CrossRef]

66. Pontius, R.G.; Schneider, L.C. Land-cover change model validation by an ROC method for the Ipswich watershed, Massachusetts, USA. Agric. Ecosyst. Environ. 2001, 85, 239-248. [CrossRef]

67. Vilar del Hoyo, L.; Martín Isabel, M.P.; Martínez Vega, F.J. Logistic regression models for human-caused wildfire risk estimation: Analysing the effect of the spatial accuracy in fire occurrence data. Eur. J. For. Res. 2011, 130, 983-996. [CrossRef]

68. Liang, X.; Liu, X.; Chen, G.; Leng, J.; Wen, Y.; Chen, G. Coupling fuzzy clustering and cellular automata based on local maxima of development potential to model urban emergence and expansion in economic development zones. Int. J. Geogr. Inf. Syst. 2020, 34, 1930-1952. [CrossRef]

69. Canqiang, Z.; Wenhua, L.; Biao, Z.; Moucheng, L. Water yield of Xitiaoxi River basin based on InVEST modeling. J. Resour. Ecol. 2012, 3, 50-54. [CrossRef]

70. Kasei, R.A.; Ampadu, B.; Sapanbil, G.S. Relationship between rainfall-runoff on the White Volta River at Pwalugu of the Volta Basin in Ghana. Environ. Earth Sci. 2013, 3, 92-99.

71. Hargreaves, J.J.; Hobbs, B.F. Optimal selection of priority development areas considering tradeoffs between hydrology and development configuration. Environ. Model. Assess. 2009, 14, 289-302. [CrossRef]

72. Office of Agricultural Economics. Agricultural Statistics of Thailand 2019. Available online: https://www.oae.go.th/assets/ portals/1/files/jounal/2563/yearbook62edit.pdf (accessed on 19 April 2021).

73. Office of the Cane and Sugar Board. Annual Report of Sugarcane Production in 2019. Available online: http://www.ocsb.go.th/ upload/journal/fileupload/923-1854.pdf (accessed on 19 April 2021).

74. Rubber Authority of Thailand. Thailand Rubber Price in 2019. Available online: https://www.raot.co.th/rubber2012/ rubberprice_yr.php (accessed on 19 April 2021).

75. Wittawatchutikul, P.; Jirasuktaveekul, W. The Technique for Court Witness: A Case of Compensation Claim due to Deforestation, Technical Report No 9/2548; National Park, Wildlife and Plant Conservation Department: Bangkok, Thailand, 2005.

76. Bank for Agriculture and Agricultural Cooperatives. Minimum Retail Rate in 2019. Available online: https://www.baac.or.th/ th/content-rate.php?content_group=9\&content_group_sub=2\&inside=1 (accessed on 19 April 2021). 